\title{
DBU-Mediated Cyclization of Acylcyclopropanecarboxylates with Amidines: Access to Polysubstituted Pyrimidines
}

\author{
Shan Wang, ${ }^{\mathrm{a}}$ Naili Luo, ${ }^{\mathrm{a}}$ Yan Li, ${ }^{\mathrm{b}}$ Cunde Wang*a \\ ${ }^{\mathrm{a}}$ School of Chemistry and Chemical Engineering, Yangzhou University, 180 Siwangting Street, \\ Yangzhou 225002, P. R. China. Fax: +86-514-8797-5244; Tel: +86-514-8797-5568; E-mail: \\ wangcd@yzu.edu.cn \\ ${ }^{\mathrm{b}}$ School of Pharmacy, Taizhou Polytechnic College, Taizhou 225300, P.R. China
}

\section{CONTENTS}

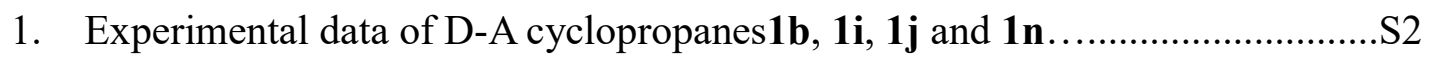

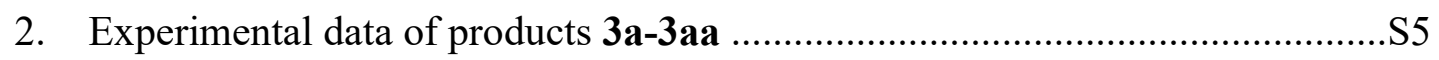

3. Copies of NMR spectroscopies of D-A cyclopropanes $\mathbf{1 b}, \mathbf{1 i}, \mathbf{1} \mathbf{j}$ and $\mathbf{1 n} \ldots \ldots \ldots . . . \mathrm{S} 15$

4. Copies of NMR spectroscopies of products 3a-3aa ...................................... 19

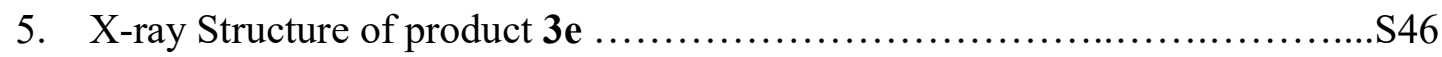




\section{Experimental Section}

\section{EXPERIMENTAL SECTION}

General Information. Unless otherwise stated, all reactions were carried out in air and using distilled solvent, without any precautions to exclude air and moisture unless otherwise noted. All reagents were purchased from commercial suppliers and used without further purification. All melting points were determined in a Mel-Tem capillary melting point apparatus. Reactions were monitored by thin-layer chromatography (TLC) carried out on commercial silica gel plates (GF 254) using UV light as a visualizing agent. Flash chromatography was performed on silica gel 60 (200-300 mesh). ${ }^{1} \mathrm{H}$ and ${ }^{13} \mathrm{C}$ spectra were recorded in DMSO- $d_{6}$ on 400 or $600 \mathrm{MHz}$ instrument in a Bruker AV-400 or -600 Spectrometer. Chemical shifts for ${ }^{1} \mathrm{H}$ NMR spectra were reported in $\delta \mathrm{ppm}$ referenced to an internal tetramethylsilane (TMS) standard. Chemical shifts for ${ }^{13} \mathrm{C}\left\{{ }^{1} \mathrm{H}\right\}$ NMR spectra were reported in parts per million relative to the center line signal of the DMSO- $d_{6}$ at $39.5 \mathrm{ppm}$ and the $\mathrm{CDCl}_{3}$ triplet at 77.0 ppm. Abbreviations for signal couplings are s, singlet; d, doublet; t, triplet; $\mathrm{m}$, multiplet. Coupling constants $(J)$ are reported in hertz and refer to apparent peak multiplications. High-resolution ESI mass spectra were obtained on a UHR-TOF maXis (ESI) mass spectrometer. X-ray crystallographic analysis was performed with a SMART APEX-II diffractometer. IR spectra were recorded on an FT-IR instrument at normal temperature with a $\mathrm{KBr}$ pellet formed by grinding the sample with $\mathrm{KBr}$ (IR grade).

Procedure for preparation of D-A cyclopropanes 1a-u

D-A cyclopropanes 1a-u were synthesized following a literature procedure method as described. ${ }^{29}$ Besides D-A cyclopropanes $1 \mathbf{b}, \mathbf{1 i}, \mathbf{1 j}$ and $\mathbf{1 n}$, other D-A cyclopropanes were known compounds.

Ethyl 2-(4-bromobenzoyl)-3-(4-chlorophenyl)-1-cyanocyclopropane-1-carboxylate (1b) 
White solid, $294 \mathrm{mg}$, yield: $68 \%$; m.p. 141.6-141.9 ${ }^{\circ} \mathrm{C}(\mathrm{EA} / \mathrm{PE})$; IR $\left(\mathrm{KBr}, \mathrm{cm}^{-1}\right): v$ 3460, 3012, 2241, 1729, 1692, 1583, 1371, 835; ${ }^{1} \mathrm{H}$ NMR (400 MHz, $\left.\mathrm{CDCl}_{3}\right) \delta(p p m)$ : $7.85(\mathrm{~d}, J=8.0 \mathrm{~Hz}, 2 \mathrm{H}), 7.65(\mathrm{~d}, J=7.6 \mathrm{HZ}, 2 \mathrm{H}), 7.39$ (d, $J=8,0 \mathrm{~Hz}, 2 \mathrm{H}), 7.30(\mathrm{~d}, J$ $=8.0 \mathrm{~Hz}, 2 \mathrm{H}), 4.14-4.11(\mathrm{~m}, 2 \mathrm{H}), 3.77(\mathrm{~d}, J=8.4 \mathrm{~Hz}, 1 \mathrm{H}), 3.58(\mathrm{~d}, J=8.8 \mathrm{~Hz}, 1 \mathrm{H})$, $1.13(\mathrm{t}, J=6.6 \mathrm{~Hz}, 3 \mathrm{H}) ;{ }^{13} \mathrm{C} \mathrm{NMR}\left(100 \mathrm{MHz}, \mathrm{CDCl}_{3}\right) \delta(p p m): 188.4,163.5,135.1$, 134.1, 132.5(2C), 130.2, 129.85, 129.82(2C), 129.6(2C), 129.3(2C), 114.9, 63.7, 39.5, 35.7, 29.5, 13.8; HR-MS (ESI) calcd. for $\mathrm{C}_{20} \mathrm{H}_{15} \mathrm{BrClNNaO}_{3}\left[(\mathrm{M}+\mathrm{Na})^{+}\right]$: 453.9822; Found: 453.9816.

Ethyl 2-(2-bromophenyl)-1-cyano-3-(4-methoxybenzoyl)cyclopropane-1-carboxylate (1i)

White solid, $303 \mathrm{mg}$, yield: $71 \%$; m.p. $142.8-142.9{ }^{\circ} \mathrm{C}(\mathrm{EA} / \mathrm{PE})$; IR $\left(\mathrm{KBr}, \mathrm{cm}^{-1}\right): v$ 3442, 3090, 2249, 1667, 1601, 1567, 1335, 848, 759; ${ }^{1} \mathrm{H}$ NMR (400 MHz, $\left.\mathrm{CDCl}_{3}\right) \delta$ (ppm): 7.55 (d, $J=8.0 \mathrm{~Hz}, 2 \mathrm{H}), 7.39$ (d, $J=8.0 \mathrm{~Hz}, 1 \mathrm{H}), 7.18(\mathrm{~d}, J=7.2 \mathrm{~Hz}, 1 \mathrm{H})$, 7.09 (t, $J=7.2 \mathrm{~Hz}, 1 \mathrm{H}), 6.98(\mathrm{t}, J=7.2 \mathrm{~Hz}, 1 \mathrm{H}), 6.76(\mathrm{~d}, J=7.6 \mathrm{~Hz}, 2 \mathrm{H}), 4.30(\mathrm{~s}, 2 \mathrm{H})$, 4.14-4.09 (m, 2H), 3.80 (s, 3H), 1.14 (t, $J=6.6 \mathrm{~Hz}, 3 \mathrm{H}) ;{ }^{13} \mathrm{C} \mathrm{NMR}\left(100 \mathrm{MHz}, \mathrm{CDCl}_{3}\right)$ $\delta(\mathrm{ppm}): 191.9,168.6,164.0,160.2,132.9,132.8,131.7,130.7,129.5(2 \mathrm{C}), 127.8$, 127.7, 125.1, 113.8(2C), 113.7, 107.9, 63.0, 55.5, 42.2, 13.7; HR-MS (ESI) calcd. for $\mathrm{C}_{21} \mathrm{H}_{18} \mathrm{BrNNaO}_{4}\left[(\mathrm{M}+\mathrm{Na})^{+}\right]:$450.0317; Found: 450.0372.

Ethyl 2-(4-chlorophenyl)-1-cyano-3-(4-methylbenzoyl)cyclopropane-1-carboxylate (1j)

White solid, $246 \mathrm{mg}$, yield: $67 \%$; m.p. $143.2-143.5^{\circ} \mathrm{C}(\mathrm{EA} / \mathrm{PE})$; IR $\left(\mathrm{KBr}, \mathrm{cm}^{-1}\right): v$ 3464, 3031, 2241, 1752, 1686, 1603, 1398, 835; ${ }^{1} \mathrm{H}$ NMR (400 MHz, $\left.\mathrm{CDCl}_{3}\right) \delta(p p m)$ : $7.90(\mathrm{~d}, J=6.8 \mathrm{~Hz}, 2 \mathrm{H}), 7.39$ (d, $J=6.8 \mathrm{~Hz}, 2 \mathrm{H}), 7.32-7.30(\mathrm{~m}, 4 \mathrm{H}), 4.13-4.09$ (m, 2H), $3.79(\mathrm{~d}, J=8.4 \mathrm{~Hz}, 1 \mathrm{H}), 3.63(\mathrm{~d}, J=8.4 \mathrm{~Hz}, 1 \mathrm{H}), 2.43(\mathrm{~s}, 3 \mathrm{H}), 1.12$ (t, $J=6.6$ $\mathrm{Hz}, 3 \mathrm{H}) ;{ }^{13} \mathrm{C} \mathrm{NMR}\left(100 \mathrm{MHz}, \mathrm{CDCl}_{3}\right) \delta(\mathrm{ppm}): 188.8,163.6,145.6,134.9,132.9$, 130.6, 129.8(2C), 129.7(2C), 129.3(2C), 128.6(2C), 115.3, 63.5, 39.9, 35.5, 29.5, 21.8, 13.8; HR-MS (ESI) calcd. for $\mathrm{C}_{21} \mathrm{H}_{18} \mathrm{ClNNaO}_{3}\left[(\mathrm{M}+\mathrm{Na})^{+}\right]$: 390.0873; Found: 390.0871 . 
Ethyl 2-(4-chlorobenzoyl)-1-cyano-3-(thiophen-3-yl)cyclopropane-1-carboxylate (1n)

White solid, $219 \mathrm{mg}$, yield: $61 \%$; m.p. 121.8-122.0 ${ }^{\circ} \mathrm{C}(\mathrm{EA} / \mathrm{PE})$; IR $\left(\mathrm{KBr}, \mathrm{cm}^{-1}\right): v$ 3427, 3099, 2243, 1738, 1690, 1588, 1397, 832; ${ }^{1} \mathrm{H}$ NMR (400 MHz, $\left.\mathrm{CDCl}_{3}\right) \delta(p p m)$ : $7.94(\mathrm{~d}, J=7.2 \mathrm{~Hz}, 2 \mathrm{H}), 7.49$ (d, $J=7.6 \mathrm{~Hz}, 2 \mathrm{H}), 7.41(\mathrm{~d}, J=7.8 \mathrm{~Hz}, 1 \mathrm{H}), 7.356$ (s, 1H), 7.13 (d, $J=7.6 \mathrm{~Hz}, 1 \mathrm{H}), 4.17-4.12(\mathrm{~m}, 2 \mathrm{H}), 3.79$ (d, $J=8.8 \mathrm{~Hz}, 1 \mathrm{H}), 3,54-3.52$ $(\mathrm{m}, 1 \mathrm{H}), 1.15(\mathrm{t}, J=6.6 \mathrm{~Hz}, 3 \mathrm{H}) ;{ }^{13} \mathrm{C} \mathrm{NMR}\left(100 \mathrm{MHz}, \mathrm{CDCl}_{3}\right) \delta(p p m): 188.2,163.7$, $140.9,133.7,132.7,129.8(2 \mathrm{C}), 129.4(2 \mathrm{C}), 127.2,126.8,124.3,115.4,63.5,40.9$, 32.3, 29.3, 13.8; HR-MS (ESI) calcd. for $\mathrm{C}_{18} \mathrm{H}_{14} \mathrm{NaClNO}_{3} \mathrm{~S}\left[(\mathrm{M}+\mathrm{Na})^{+}\right]$: 382.0281 ; Found: 382.0271. 
General Procedure for the Synthesis of 2-amino-5-aryl-4-benzylfuran-3-carbonitriles 3a-3aa.

To the mixture of substituted 1-cyanocyclopropane-1-carboxylates 1a-u (1.0 mmol), and amidines 2a-d (1.2 mmol) in $\mathrm{MeCN}(15 \mathrm{~mL})$ was added DBU (152 $\mathrm{mg}, 1.0 \mathrm{mmol}$ ), and the resulting mixture was slowly warmed up to reflux and stirred sequentially for $8.5-12 \mathrm{~h}$ until full conversion of the cyclopropanes was achieved (monitored by TLC, Hexanes/EtOAc, 3/1). Upon completion, removal of solvent $\mathrm{MeCN}$, the reaction mixture was diluted with $15 \mathrm{~mL}$ of $\mathrm{H}_{2} \mathrm{O}$ and extracted with dichloromethane $(10 \mathrm{~mL} \mathrm{X} \mathrm{2).} \mathrm{The} \mathrm{combined} \mathrm{dichloromethane}$ layers were washed with water $(10 \mathrm{~mL})$ and brine $(15 \mathrm{~mL})$, and dried over anhydrous sodium sulphate, and concentrated under reduced pressure to yield a yellowish oily product. The crude product was purified by column chromatography (EtOAc/hexanes, 1/10, silica gel) to provide the desired products 3a-aa.

4-(2-chlorobenzyl)-2-phenyl-6-(p-tolyl)pyrimidine (3a)

White solid, $277.4 \mathrm{mg}$, yield: $75 \%$; m.p. $120.3-120.5{ }^{\circ} \mathrm{C}(\mathrm{EA} / \mathrm{PE})$; IR $\left(\mathrm{KBr}, \mathrm{cm}^{-1}\right): v$ $3455,3060,1575,1530,1489,1367,1085,1010,835,785 ;{ }^{1} \mathrm{H}$ NMR (400 MHz, $\left.\mathrm{CDCl}_{3}\right) \delta(p p m): 8.43-8.42(\mathrm{~m}, 2 \mathrm{H}), 8.16(\mathrm{~d}, J=7.6 \mathrm{~Hz}, 2 \mathrm{H}), 7.80(\mathrm{~s}, 1 \mathrm{H}), 7.50-7.45$ $(\mathrm{m}, 5 \mathrm{H}), 7.36(\mathrm{~d}, J=7.6 \mathrm{~Hz}, 2 \mathrm{H}), 7.33-7.30(\mathrm{~m}, 2 \mathrm{H}), 4.33(\mathrm{~s}, 2 \mathrm{H}), 2.37(\mathrm{~s}, 3 \mathrm{H}) ;{ }^{13} \mathrm{C}$ NMR $\left(150 \mathrm{MHz}, \mathrm{CDCl}_{3}\right) \delta(\mathrm{ppm}): 168.8,163.2,163.1,141.1,137.3,135.8,133.5$, $133.5,131.9,130.8,129.6(2 \mathrm{C}), 129.3,128.7,128.6(2 \mathrm{C}), 127.8(2 \mathrm{C}), 127.3,126.9(2 \mathrm{C})$, 113.5, 40.9, 21.0; HR-MS (ESI) calcd. for $\mathrm{C}_{24} \mathrm{H}_{20} \mathrm{ClN}_{2}\left[(\mathrm{M}+\mathrm{H})^{+}\right]$: 371.1310; Found: 371.1310.

4-(4-bromophenyl)-6-(4-chlorobenzyl)-2-phenylpyrimidine (3b)

White solid, $282.1 \mathrm{mg}$, yield: $65 \%$; m.p. $109.3-109.5{ }^{\circ} \mathrm{C}(\mathrm{EA} / \mathrm{PE})$; IR $\left(\mathrm{KBr}, \mathrm{cm}^{-1}\right): v$ 3692, 3061, 1574, 1529, 1484, 1371, 1074, 1003, 822, 747; ${ }^{1} \mathrm{H}$ NMR (400 MHz, 
$\left.\mathrm{CDCl}_{3}\right) \delta(p p m): 8.47-8.45(\mathrm{~m}, 2 \mathrm{H}), 8.22(\mathrm{~d}, J=7.2 \mathrm{~Hz}, 2 \mathrm{H}), 7.96(\mathrm{~s}, 1 \mathrm{H}), 7,74(\mathrm{~d}, J=$ $7.6 \mathrm{~Hz}, 2 \mathrm{H}), 7.52-7.51(\mathrm{~m}, 3 \mathrm{H}), 7.43$ (d, $J=7.6 \mathrm{~Hz}, 2 \mathrm{H}), 7.35$ (d, $J=7.6 \mathrm{~Hz}, 2 \mathrm{H})$, 4.17 (s, 2H); $\left.{ }^{13} \mathrm{C} \mathrm{NMR} \mathrm{(150} \mathrm{MHz,} \mathrm{CDCl}_{3}\right) \delta($ ppm $): 170.1,163.3,162.3,137.3,137.1$, $135.4,132.0(2 \mathrm{C}), 131.3,131.0(2 \mathrm{C}), 130.9,129.1(2 \mathrm{C}), 128.6(2 \mathrm{C}), 128.4(2 \mathrm{C})$, 127.9(2C), 124.9, 113.9, 42.7; HR-MS (ESI) calcd. for $\mathrm{C}_{23} \mathrm{H}_{17} \mathrm{BrClN}_{2}\left[(\mathrm{M}+\mathrm{H})^{+}\right]$: 435.0258; Found: 435.0286.

4-(2-chlorobenzyl)-6-(4-chlorophenyl)-2-phenylpyrimidine (3c)

White solid, $276.9 \mathrm{mg}$, yield: 71\%; m.p. 146.6-146.9 ${ }^{\circ} \mathrm{C}(\mathrm{EA} / \mathrm{PE})$; IR $\left(\mathrm{KBr}, \mathrm{cm}^{-1}\right): v$ 3728, 3052, 1574, 1532, 1483, 1370, 1089, 1014, 829, 754; ${ }^{1} \mathrm{H}$ NMR (400 MHz, $\left.\mathrm{CDCl}_{3}\right) \delta(p p m): 8.43-8.41(\mathrm{~m}, 2 \mathrm{H}), 8.29(\mathrm{~d}, J=8.0 \mathrm{~Hz}, 2 \mathrm{H}), 7.87(\mathrm{~s}, 1 \mathrm{H}), 7.62(\mathrm{~d}, J=$ 8.0 Hz, 2H), 7.51-7.50 (m, 4H), 7.47 (d, J=7.6 Hz, 1H), 7.35-7.28 (m, 2H), 4.35 (s, $2 \mathrm{H}) ;{ }^{13} \mathrm{C} \mathrm{NMR}\left(150 \mathrm{MHz}, \mathrm{CDCl}_{3}\right) \delta(\mathrm{ppm}): 169.2,163.2,162.1,137.1,136.0,135.7$, 135.1, 133.5, 132.0, 130.9, 129.3, 129.1(2C), 128.9(2C), 128.7, 128.6(2C), 127.8(2C), 127.3, 114.0, 40.9; HR-MS (ESI) calcd. for $\mathrm{C}_{23} \mathrm{H}_{17} \mathrm{Cl}_{2} \mathrm{~N}_{2}\left[(\mathrm{M}+\mathrm{H})^{+}\right]$: 391.0763 ; Found: 391.0758 .

4-(2-bromobenzyl)-6-(4-bromophenyl)-2-phenylpyrimidine (3d)

White solid, $302.3 \mathrm{mg}$, yield: $63 \%$; m.p. 160.2-160.3 ${ }^{\circ} \mathrm{C}(\mathrm{EA} / \mathrm{PE})$; IR $\left(\mathrm{KBr}, \mathrm{cm}^{-1}\right): v$ 3455, 3052, 1574, 1529, 1477, 1369, 1067, 1009, 825, 753; ${ }^{1} \mathrm{H}$ NMR (400 MHz, $\left.\mathrm{CDCl}_{3}\right) \delta(p p m): 8.43-8.42(\mathrm{~m}, 2 \mathrm{H}), 8.21(\mathrm{~d}, J=8.0 \mathrm{~Hz}, 2 \mathrm{H}), 7.85(\mathrm{~s}, 1 \mathrm{H}), 7.76(\mathrm{~d}, J=$ $8.0 \mathrm{~Hz}, 2 \mathrm{H}), 7.64$ (d, $J=7.6 \mathrm{~Hz}, 1 \mathrm{H}), 7.51-7.48$ (m, 4H), 7.38 (dd, $J=7.6,7.2 \mathrm{~Hz}$, 1H), $7.22(\mathrm{dd}, J=7.6,7.2 \mathrm{~Hz}, 1 \mathrm{H}), 4.36(\mathrm{~s}, 2 \mathrm{H}) ;{ }^{13} \mathrm{C} \mathrm{NMR}\left(150 \mathrm{MHz}, \mathrm{CDCl}_{3}\right) \delta$ (ppm): 169.2, 163.2, 162.1, 137.4, 137.1, 135.5, 132.6, 132.1(2C), 132.0, 130.9, 129.1(2C), 128.9, 128.6(2C), 127.9, 127.8(2C), 124.9, 124.4, 114.0, 43.4; HR-MS (ESI) calcd. for $\mathrm{C}_{23} \mathrm{H}_{17} \mathrm{Br}_{2} \mathrm{~N}_{2}\left[(\mathrm{M}+\mathrm{H})^{+}\right]$: 480.9733 ; Found: 480.9788.

\section{4-(4-bromobenzyl)-2,6-bis(4-chlorophenyl)pyrimidine (3e)}

Yellow solid, $388.4 \mathrm{mg}$, yield: 83\%; m.p. $154.7-154.9{ }^{\circ} \mathrm{C}(\mathrm{EA} / \mathrm{PE})$; IR $\left(\mathrm{KBr}, \mathrm{cm}^{-1}\right)$ : $v$ $3444,2892,1584,1533,1486,1373,1170,1087,834,785 ;{ }^{1} \mathrm{H}$ NMR (400 MHz, 
$\left.\mathrm{CDCl}_{3}\right) \delta(\mathrm{ppm}): 8.46(\mathrm{~d}, J=8.0 \mathrm{~Hz}, 2 \mathrm{H}), 8.31(\mathrm{~d}, J=8.0 \mathrm{~Hz}, 2 \mathrm{H}), 8.01(\mathrm{~s}, 1 \mathrm{H}), 7.63$ (d, $J=8.0 \mathrm{~Hz}, 2 \mathrm{H}), 7.59$ (d, $J=8 \mathrm{~Hz}, 2 \mathrm{H}), 7.49$ (d, $J=7.6 \mathrm{~Hz}, 2 \mathrm{H}), 7.36$ (d, $J=8 \mathrm{~Hz}$, 2H), $4.17(\mathrm{~s}, 2 \mathrm{H}) ;{ }^{13} \mathrm{C} \mathrm{NMR}\left(600 \mathrm{MHz}, \mathrm{CDCl}_{3}\right) \delta(\mathrm{ppm}): 170.2,162.3,137.6,136.1$, $135.9,135.8,134.9,131.41(2 \mathrm{C}), 131.38(2 \mathrm{C}), 129.6(2 \mathrm{C}), 129.1(2 \mathrm{C}), 128.9(2 \mathrm{C})$, 128.8(2C), 119.8, 114.3, 104.5, 42.7; HR-MS (ESI) calcd. for $\mathrm{C}_{23} \mathrm{H}_{16} \mathrm{BrCl}_{2} \mathrm{~N}_{2}$ $\left[(\mathrm{M}+\mathrm{H})^{+}\right]:$468.9868; Found: 468.9866.

4-(4-bromophenyl)-6-(4-chlorobenzyl)-2-(4-chlorophenyl)pyrimidine (3f)

Yellow solid, $397.7 \mathrm{mg}$, yield: $85 \%$; m.p. $162.4-162.7{ }^{\circ} \mathrm{C}(\mathrm{EA} / \mathrm{PE})$; IR $\left(\mathrm{KBr}, \mathrm{cm}^{-1}\right): v$ 3444, 2886, 1581, 1530, 1484, 1371, 1170, 1081, 829, 783; ${ }^{1} \mathrm{H}$ NMR (400 MHz, $\left.\mathrm{CDCl}_{3}\right) \delta(\mathrm{ppm}): 8.45(\mathrm{~d}, J=6.8 \mathrm{~Hz}, 2 \mathrm{H}), 8.22(\mathrm{~d}, J=7.2 \mathrm{~Hz}, 2 \mathrm{H}), 7.99(\mathrm{~s}, 1 \mathrm{H}), 7.75$ $(\mathrm{d}, J=7.2 \mathrm{~Hz}, 2 \mathrm{H}), 7.58$ (d, $J=7.2 \mathrm{~Hz}, 2 \mathrm{H}), 7.42$ (d, $J=7.2 \mathrm{~Hz}, 2 \mathrm{H}), 7.36$ (d, $J=6.8$ $\mathrm{Hz}, 2 \mathrm{H}), 4.18(\mathrm{~s}, 2 \mathrm{H}) ;{ }^{13} \mathrm{C} \mathrm{NMR}\left(150 \mathrm{MHz}, \mathrm{CDCl}_{3}\right) \delta(\mathrm{ppm}): 170.2,162.4,162.3$, 137.2 , 135.9, 135.8, 135.3, 132.0(2C), 131.3, 130.9(2C), 129.6(2C), 129.1(2C), 128.8(2C), 128.5(2C), 125.0, 114.3, 42.61; HR-MS (ESI) calcd. for $\mathrm{C}_{23} \mathrm{H}_{16} \mathrm{BrCl}_{2} \mathrm{~N}_{2}$ $\left[(\mathrm{M}+\mathrm{H})^{+}\right]:$468.9868; Found: 468.9876

\section{4-(2-bromobenzyl)-2,6-bis(4-chlorophenyl)pyrimidine (3g)}

White solid, $369.7 \mathrm{mg}$, yield: $79 \%$; m.p. $141.1-141.3{ }^{\circ} \mathrm{C}(\mathrm{EA} / \mathrm{PE})$; IR $\left(\mathrm{KBr}, \mathrm{cm}^{-1}\right): v$ 3451, 3063, 1579, 1528, 1483, 1368, 1083, 1010, 830, 740; ${ }^{1} \mathrm{H}$ NMR (400 MHz, $\left.\mathrm{CDCl}_{3}\right) \delta(p p m): 8.41(\mathrm{~d}, J=8.0 \mathrm{~Hz}, 2 \mathrm{H}), 8.27(\mathrm{~d}, J=8.0 \mathrm{~Hz}, 2 \mathrm{H}), 7.86(\mathrm{~s}, 1 \mathrm{H}), 7.62$ $(\mathrm{dd}, J=8.0,8.0 \mathrm{~Hz}, 3 \mathrm{H}), 7.56(\mathrm{~d}, J=8.4 \mathrm{~Hz}, 2 \mathrm{H}), 7.48$ (d, $J=7.6 \mathrm{~Hz}, 1 \mathrm{H}), 7.37$ (dd, $J=7.2,7.6 \mathrm{~Hz}, 1 \mathrm{H}), 7.22(\mathrm{dd}, J=8.4,7.2 \mathrm{~Hz}, 1 \mathrm{H}), 4.35(\mathrm{~s}, 2 \mathrm{H}) ;{ }^{13} \mathrm{C} \mathrm{NMR}(150 \mathrm{MHz}$, $\left.\mathrm{CDCl}_{3}\right) \delta(\mathrm{ppm}): 169.9,162.2,137.3,136.1,135.9,135.8,134.9,132.6,132.1$, 129.6(2C), 129.1(2C), 128.9, 128.8(2C), 128.7(2C), 127.9, 124.4, 114.3, 104.5, 43.4; HR-MS (ESI) calcd. for $\mathrm{C}_{23} \mathrm{H}_{16} \mathrm{BrCl}_{2} \mathrm{~N}_{2}\left[(\mathrm{M}+\mathrm{H})^{+}\right]$: 468.9868 ; Found: 468.9869

\section{4-(3-chlorobenzyl)-2,6-bis(4-chlorophenyl)pyrimidine (3h)}

White solid, $326.5 \mathrm{mg}$, yield: $77 \%$; m.p. $119.9-120.1{ }^{\circ} \mathrm{C}(\mathrm{EA} / \mathrm{PE})$; IR $\left(\mathrm{KBr}, \mathrm{cm}^{-1}\right): v$ $3455,3063,1580,1532,1485,1368,1086,1009,834,785 ;{ }^{1} \mathrm{H}$ NMR (400 MHz, 
$\left.\mathrm{CDCl}_{3}\right) \delta(p p m): 8.46(\mathrm{~d}, J=8.4 \mathrm{~Hz}, 2 \mathrm{H}), 8.31(\mathrm{~d}, J=8.4 \mathrm{~Hz}, 2 \mathrm{H}), 8.04(\mathrm{~s}, 1 \mathrm{H}), 7.63$ $(\mathrm{d}, J=8.4 \mathrm{~Hz}, 2 \mathrm{H}), 7.59(\mathrm{~d}, J=8.8 \mathrm{~Hz}, 2 \mathrm{H}), 7.50(\mathrm{~s}, 1 \mathrm{H}), 7.38(\mathrm{~d}, J=7.6 \mathrm{~Hz}, 1 \mathrm{H})$, $7.34(\mathrm{dd}, J=8.0,7.6 \mathrm{~Hz}, 1 \mathrm{H}), 7.28(\mathrm{~d}, J=8.0 \mathrm{~Hz}, 2 \mathrm{H}), 4.20(\mathrm{~s}, 2 \mathrm{H}) ;{ }^{13} \mathrm{C}$ NMR $(150$ $\left.\mathrm{MHz}, \mathrm{CDCl}_{3}\right) \delta(\mathrm{ppm}): 169.9,162.3,162.3,140.6,136.1,135.9,135.8,134.9,133.0$, 130.4, 129.6(2C), 129.1(2C), 128.9(2C), 128.8(2C), 127.9, 126.6, 117.5, 114.4, 42.8; HR-MS (ESI) calcd. for $\mathrm{C}_{23} \mathrm{H}_{16} \mathrm{Cl}_{3} \mathrm{~N}_{2}\left[(\mathrm{M}+\mathrm{H})^{+}\right]$: 425.0374 ; Found: 425.0376 .

4-(3-chlorobenzyl)-2-(4-chlorophenyl)-6-phenylpyrimidine (3i)

White solid, $253.5 \mathrm{mg}$, yield: $65 \%$; m.p. $83.2-83.5{ }^{\circ} \mathrm{C}(\mathrm{EA} / \mathrm{PE})$; IR $\left(\mathrm{KBr}, \mathrm{cm}^{-1}\right): v$ 3452, 3059, 1580, 1538, 1485, 1373, 1089, 1012, 845, 772; ${ }^{1} \mathrm{H}$ NMR (400 MHz, $\left.\mathrm{CDCl}_{3}\right) \delta(\mathrm{ppm}): 8.47(\mathrm{~d}, J=8 \mathrm{~Hz}, 2 \mathrm{H}), 8.28-8.27(\mathrm{~m}, 2 \mathrm{H}), 8.01(\mathrm{~s}, 1 \mathrm{H}), 7.60-7.56(\mathrm{~m}$, 5H), $7.50(\mathrm{~s}, 1 \mathrm{H}), 7.38(\mathrm{~d}, J=7.6 \mathrm{~Hz}, 1 \mathrm{H}), 7.34(\mathrm{dd}, J=8.0,7.2 \mathrm{~Hz}, 1 \mathrm{H}), 7.28(\mathrm{~d}, J=$ $7.6 \mathrm{~Hz}, 1 \mathrm{H}), 4,20(\mathrm{~s}, 2 \mathrm{H}) ;{ }^{13} \mathrm{C} \mathrm{NMR}\left(150 \mathrm{MHz}, \mathrm{CDCl}_{3}\right) \delta($ ppm): 169.7, 163.5, 162.2, $140.6,136.1,135.9,135.8,134.9,131.3,130.4,129.6(2 \mathrm{C}), 129.0(2 \mathrm{C}), 128.9$, 128.8(2C), 127.9, 127.1(2C), 126.6, 114.4, 42.8; HR-MS (ESI) calcd. for $\mathrm{C}_{23} \mathrm{H}_{17} \mathrm{Cl}_{2} \mathrm{~N}_{2}$ $\left[(\mathrm{M}+\mathrm{H})^{+}\right]$: 391.0763; Found: 391.0762.

4-(2-bromobenzyl)-2-(4-chlorophenyl)-6-(4-methoxyphenyl)pyrimidine (3j)

White solid, $320.2 \mathrm{mg}$, yield: $69 \%$; m.p. $145.9-146.2{ }^{\circ} \mathrm{C}(\mathrm{EA} / \mathrm{PE})$; IR $\left(\mathrm{KBr}, \mathrm{cm}^{-1}\right): v$ 3450, 3056, 1579, 1526, 1487, 1373, 1090, 1024, 837, 786; ${ }^{1} \mathrm{H}$ NMR (400 MHz, $\left.\mathrm{CDCl}_{3}\right) \delta(\mathrm{ppm}): 8.42(\mathrm{~d}, J=8.4 \mathrm{~Hz}, 2 \mathrm{H}), 8.21(\mathrm{~d}, J=8.8 \mathrm{~Hz}, 2 \mathrm{H}), 7.75(\mathrm{~s}, 1 \mathrm{H}), 7.64$ $(\mathrm{d}, J=8.0 \mathrm{~Hz}, 1 \mathrm{H}), 7.56(\mathrm{~d}, J=8.4 \mathrm{~Hz}, 2 \mathrm{H}), 7.48(\mathrm{~d}, J=7.6 \mathrm{~Hz}, 1 \mathrm{H}), 7.37(\mathrm{~m}, 1 \mathrm{H})$, $7.21(\mathrm{dd}, J=7.6,7.6 \mathrm{~Hz}, 1 \mathrm{H}), 7.09(\mathrm{~d}, J=8.4 \mathrm{~Hz}, 2 \mathrm{H}), 4.32(\mathrm{~s}, 2 \mathrm{H}), 3.82(\mathrm{~s}, 3 \mathrm{H}) ;{ }^{13} \mathrm{C}$ NMR $\left(150 \mathrm{MHz}, \mathrm{CDCl}_{3}\right) \delta(\mathrm{ppm}): 168.6,162.9,162.0,161.9,137.5,136.2,135.7$, 132.6(2C), 132.0, 129.5(2C), 128.9, 128.6(2C), 128.3, 127.9, 124.4, 114.5(2C), 113.3, 104.5, 55.4, 43.3; HR-MS (ESI) calcd. for $\mathrm{C}_{24} \mathrm{H}_{19} \mathrm{BrClN}_{2} \mathrm{O}\left[(\mathrm{M}+\mathrm{H})^{+}\right]$: 465.0364; Found: 465.0373 .

4-(4-chlorobenzyl)-2-(4-chlorophenyl)-6-(p-tolyl)pyrimidine (3k)

White solid, $286.9 \mathrm{mg}$, yield: 71\%; m.p. $111.5-111.7^{\circ} \mathrm{C}(\mathrm{EA} / \mathrm{PE})$; IR $\left(\mathrm{KBr}, \mathrm{cm}^{-1}\right): v$ 
3455, 3031, 1577, 1531, 1489, 1366, 1085, 1009, 822, 783; ${ }^{1} \mathrm{H}$ NMR (400 MHz, $\left.\mathrm{CDCl}_{3}\right) \delta(\mathrm{ppm}): 8.45(\mathrm{~d}, J=8.4 \mathrm{~Hz}, 2 \mathrm{H}), 8.16(\mathrm{~d}, J=8.4 \mathrm{~Hz}, 2 \mathrm{H}), 7.92(\mathrm{~s}, 1 \mathrm{H}), 7.56$ (d, $J=8.4 \mathrm{~Hz}, 2 \mathrm{H}), 7.42(\mathrm{~d}, J=8.4 \mathrm{~Hz}, 2 \mathrm{H}), 7.35$ (d, $J=8.4 \mathrm{~Hz}, 4 \mathrm{H}), 4.15(\mathrm{~s}, 2 \mathrm{H})$, $2.36(\mathrm{~s}, 3 \mathrm{H}) ;{ }^{13} \mathrm{C} \mathrm{NMR}\left(150 \mathrm{MHz}, \mathrm{CDCl}_{3}\right) \delta(\mathrm{ppm}): 169.7,163.4,162.2,141.3,137.3$, $136.2,135.7,133.3,131.2,130.9(2 \mathrm{C}), 129.6(2 \mathrm{C}), 129.5(2 \mathrm{C}), 128.7(2 \mathrm{C}), 128.5(2 \mathrm{C})$, 127.0(2C), 113.8, 42.6, 21.0; HR-MS (ESI) calcd. for $\mathrm{C}_{24} \mathrm{H}_{19} \mathrm{Cl}_{2} \mathrm{~N}_{2}\left[(\mathrm{M}+\mathrm{H})^{+}\right]$: 405.0920; Found: 405.0912.

4-(4-chlorobenzyl)-2-(4-chlorophenyl)-6-(4-methoxyphenyl)pyrimidine (31)

White solid, $302.5 \mathrm{mg}$, yield: $72 \%$; m.p. $121.9-122.1{ }^{\circ} \mathrm{C}(\mathrm{EA} / \mathrm{PE}) ; \mathrm{IR}\left(\mathrm{KBr}, \mathrm{cm}^{-1}\right): v$ 3450, 3044, 1576, 1520, 1487, 1362, 1084, 1016, 831, 783; ${ }^{1} \mathrm{H}$ NMR (400 MHz, $\left.\mathrm{CDCl}_{3}\right) \delta(\mathrm{ppm}): 8.44(\mathrm{~d}, J=8.4 \mathrm{~Hz}, 2 \mathrm{H}), 8.23(\mathrm{~d}, J=8.4 \mathrm{~Hz}, 2 \mathrm{H}), 7.86(\mathrm{~s}, 1 \mathrm{H}), 7.55$ (d, $J=8.4 \mathrm{~Hz}, 2 \mathrm{H}), 7.41(\mathrm{~d}, J=8.4 \mathrm{~Hz}, 2 \mathrm{H}), 7.35(\mathrm{~d}, J=8.4 \mathrm{~Hz}, 2 \mathrm{H}), 7.07$ (d, $J=8.8$ $\mathrm{Hz}, 2 \mathrm{H}), 4.13(\mathrm{~s}, 2 \mathrm{H}), 3.81(\mathrm{~s}, 3 \mathrm{H}) ;{ }^{13} \mathrm{C} \mathrm{NMR}\left(150 \mathrm{MHz}, \mathrm{CDCl}_{3}\right) \delta(p p m): 169.4$, $163.1,162.1,161.6,137.3,136.2,135.6,131.2,130.9(2 \mathrm{C}), 129.5(2 \mathrm{C}), 128.7(2 \mathrm{C})$, 128.6(2C), 128.4(2C), 128.3, 114.3(2C), 113.2, 55.3, 42.6; HR-MS (ESI) calcd. for $\mathrm{C}_{24} \mathrm{H}_{19} \mathrm{Cl}_{2} \mathrm{~N}_{2} \mathrm{O}\left[(\mathrm{M}+\mathrm{H})^{+}\right]: 421.0869$; Found: 421.0867.

2-(4-chlorophenyl)-4-(4-methylbenzyl)-6-phenylpyrimidine (3 m)

Yellow solid, $244.3 \mathrm{mg}$, yield: 66\%; m.p. 120.9-121.2 ${ }^{\circ} \mathrm{C}(\mathrm{EA} / \mathrm{PE}) ; \mathrm{IR}\left(\mathrm{KBr}, \mathrm{cm}^{-1}\right): v$ 3455, 3021, 1579, 1528, 1424, 1355, 1083, 1009, 838, 763; ${ }^{1} \mathrm{H}$ NMR (400 MHz, $\left.\mathrm{CDCl}_{3}\right) \delta(p p m): 8.48(\mathrm{~d}, J=7.6 \mathrm{~Hz}, 2 \mathrm{H}), 8.25-8.23(\mathrm{~m}, 2 \mathrm{H}), 7.91(\mathrm{~s}, 1 \mathrm{H}), 7.58-7.54$ (m, 5H), 7.27 (d, $J=7.2 \mathrm{~Hz}, 2 \mathrm{H}), 7.10$ (d, $J=7.2 \mathrm{~Hz}, 2 \mathrm{H}), 4,12(\mathrm{~s}, 2 \mathrm{H}), 2.22(\mathrm{~s}, 3 \mathrm{H})$; ${ }^{13} \mathrm{C} \mathrm{NMR}\left(150 \mathrm{MHz}, \mathrm{CDCl}_{3}\right) \delta(\mathrm{ppm}): 170.7,163.4,162.2,136.2,136.1,135.7,135.6$, 135.2, 131.2, 129.6(2C), 129.1(2C), 129.0(2C), 128.9(2C), 128.7(2C), 127.1(2C), 114.2, 43.1, 20.6; HR-MS (ESI) calcd. for $\mathrm{C}_{24} \mathrm{H}_{20} \mathrm{ClN}_{2}\left[(\mathrm{M}+\mathrm{H})^{+}\right]$: 371.1315; Found: 371.1303

4-(4-bromophenyl)-6-(3-chlorobenzyl)-2-(4-chlorophenyl)pyrimidine (3n)

White solid, $346.3 \mathrm{mg}$, yield: $74 \%$; m.p. $153.6-153.8{ }^{\circ} \mathrm{C}(\mathrm{EA} / \mathrm{PE})$; IR $\left(\mathrm{KBr}, \mathrm{cm}^{-1}\right): v$ 
3450, 3060, 1580, 1528, 1424, 1355, 1080, 1007, 832, 785; ${ }^{1} \mathrm{H}$ NMR (400 MHz, $\left.\mathrm{CDCl}_{3}\right) \delta(p p m): 8.46(\mathrm{~d}, J=8.4 \mathrm{~Hz}, 2 \mathrm{H}), 8.23(\mathrm{~d}, J=8.4 \mathrm{~Hz}, 2 \mathrm{H}), 8.03(\mathrm{~s}, 1 \mathrm{H}), 7.76$ $(\mathrm{d}, J=8.4 \mathrm{~Hz}, 2 \mathrm{H}), 7.58(\mathrm{~d}, J=8.4 \mathrm{~Hz}, 2 \mathrm{H}), 7,49(\mathrm{~s}, 1 \mathrm{H}), 7.38-7.31(\mathrm{~m}, 2 \mathrm{H}), 7.28(\mathrm{~d}$, $J=7.2 \mathrm{~Hz}, 1 \mathrm{H}), 4.19(\mathrm{~s}, 2 \mathrm{H}) ;{ }^{13} \mathrm{C} \mathrm{NMR}\left(150 \mathrm{MHz}, \mathrm{CDCl}_{3}\right) \delta(\mathrm{ppm}): 169.9,162.4$, $162.3,140.6,135.9,135.8,135.2,133.0,132.0(2 \mathrm{C}), 130.4,129.6(2 \mathrm{C}), 129.1(2 \mathrm{C})$, 128.9, 128.8(2C), 127.9, 126.6, 125.1, 114.3, 42.8; HR-MS (ESI) calcd. for $\mathrm{C}_{23} \mathrm{H}_{16} \mathrm{BrCl}_{2} \mathrm{~N}_{2}\left[(\mathrm{M}+\mathrm{H})^{+}\right]$: 468.9868 ; Found: 468.9872.

4-(2-chlorobenzyl)-2-(4-chlorophenyl)-6-(p-tolyl)pyrimidine (3o)

White solid, $278.8 \mathrm{mg}$, yield: $69 \%$; m.p. $123.3-123.4{ }^{\circ} \mathrm{C}(\mathrm{EA} / \mathrm{PE}) ; \mathrm{IR}\left(\mathrm{KBr}, \mathrm{cm}^{-1}\right): v$ 3455, 3061, 1577, 1529, 1487, 1367, 1087, 1013, 830, 780; ${ }^{1} \mathrm{H}$ NMR (400 MHz, $\left.\mathrm{CDCl}_{3}\right) \delta(\mathrm{ppm}): 8.41(\mathrm{~d}, J=7.6 \mathrm{~Hz}, 2 \mathrm{H}), 8.44(\mathrm{~d}, J=7.6 \mathrm{~Hz}, 2 \mathrm{H}), 7.80(\mathrm{~s}, 1 \mathrm{H}), 7.56$ (d, $J=7.6 \mathrm{~Hz}, 2 \mathrm{H}), 7.47$ (dd, $J=8.0,8.4 \mathrm{~Hz}, 2 \mathrm{H}), 7.35$ (d, $J=7.6 \mathrm{~Hz}, 2 \mathrm{H}), 7.32-7.27$ $(\mathrm{m}, 2 \mathrm{H}), 4.32(\mathrm{~s}, 2 \mathrm{H}), 2.36(\mathrm{~s}, 3 \mathrm{H}) ;{ }^{13} \mathrm{C} \mathrm{NMR}\left(600 \mathrm{MHz}, \mathrm{CDCl}_{3}\right) \delta(p p m): 168.9$, $163.3,162.1,141.3,136.14,136.07,135.71,135.69,133.5,133.3,131.9,129.6(2 \mathrm{C})$, 129.5(2C), 129.3, 128.7(2C), 127.4, 126.9(2C), 113.8, 40.9, 21.0; HR-MS (ESI) calcd. for $\mathrm{C}_{24} \mathrm{H}_{19} \mathrm{Cl}_{2} \mathrm{~N}_{2}\left[(\mathrm{M}+\mathrm{H})^{+}\right]$: 405.0920; Found: 405.0917.

2,4-bis(4-chlorophenyl)-6-(thiophen-3-ylmethyl)pyrimidine (3p)

Yellow solid, $229.7 \mathrm{mg}$, yield: 58\%; m.p. 144.6-144.8 ${ }^{\circ} \mathrm{C}(\mathrm{EA} / \mathrm{PE}) ; \mathrm{IR}\left(\mathrm{KBr}, \mathrm{cm}^{-1}\right): v$ 3438, 2890, 1581, 1530, 1487, 1363, 1068, 1015, 831, 785; ${ }^{1} \mathrm{H}$ NMR (400 MHz, $\left.\mathrm{CDCl}_{3}\right) \delta(p p m): 8.48(\mathrm{~d}, J=7.2 \mathrm{~Hz}, 2 \mathrm{H}), 8.29(\mathrm{~d}, J=7.2 \mathrm{~Hz}, 2 \mathrm{H}), 7.96(\mathrm{~s}, 1 \mathrm{H})$, 7,62-7.58 (m, 4H), 7.49-7.47 (m, 1H), 7.37 (s, 1H), 7.15-7.14 (m, 1H), 4.19 (s, 2H); ${ }^{13} \mathrm{C}$ NMR (600 MHz, $\left.\mathrm{CDCl}_{3}\right) \delta(p p m): 170.3,162.3,162.2,137.7,136.1,136.0,135.8$, 134.9, 129.6(2C), 129.1(2C), 128.9(2C), 128.8(2C), 128.6, 126.3, 122.6, 114.1, 38.2; HR-MS (ESI) calcd. for $\mathrm{C}_{21} \mathrm{H}_{15} \mathrm{Cl}_{2} \mathrm{~N}_{2} \mathrm{~S}\left[(\mathrm{M}+\mathrm{H})^{+}\right]$: 397.0328 ; Found: 397.0343 .

4-(4-chlorobenzyl)-2,6-bis(4-chlorophenyl)pyrimidine (3q)

Yellow solid, $334.9 \mathrm{mg}$, yield: 79\%; m.p. 157.8-158.1 ${ }^{\circ} \mathrm{C}(\mathrm{EA} / \mathrm{PE})$; IR $\left(\mathrm{KBr}, \mathrm{cm}^{-1}\right): v$ $3381,2975,1582,1531,1485,1371,1085,1009,830,783 ;{ }^{1} \mathrm{H}$ NMR (400 MHz, 
$\left.\mathrm{CDCl}_{3}\right) \delta(\mathrm{ppm}): 8.46(\mathrm{~d}, J=8.0 \mathrm{~Hz}, 2 \mathrm{H}), 8.30(\mathrm{~d}, J=8.4 \mathrm{~Hz}, 2 \mathrm{H}), 8.01(\mathrm{~s}, 1 \mathrm{H}), 7.62$ $(\mathrm{d}, J=8.4 \mathrm{~Hz}, 2 \mathrm{H}), 7.58(\mathrm{~d}, J=8.4 \mathrm{~Hz}, 2 \mathrm{H}), 7.42(\mathrm{~d}, J=8.0 \mathrm{~Hz}, 2 \mathrm{H}), 7.36(\mathrm{~d}, J=8.0$ $\mathrm{Hz}, 2 \mathrm{H}), 4.18(\mathrm{~s}, 2 \mathrm{H}) ;{ }^{13} \mathrm{C} \mathrm{NMR}\left(150 \mathrm{MHz}, \mathrm{CDCl}_{3}\right) \delta(\mathrm{ppm}): 170.2,162.3,137.2$, $136.1,135.9,135.8,134.9,131.3,130.9(2 \mathrm{C}), 129.6(2 \mathrm{C}), 129.1(2 \mathrm{C}), 128.9(2 \mathrm{C})$, 128.8(2C), 128.5(2C), 114.3, 104.5, 42.6; HR-MS (ESI) calcd. for $\mathrm{C}_{23} \mathrm{H}_{16} \mathrm{Cl}_{3} \mathrm{~N}_{2}$ $\left[(\mathrm{M}+\mathrm{H})^{+}\right]:$425.0374; Found: 425.0389 .

4-(4-bromobenzyl)-6-(4-bromophenyl)-2-(4-chlorophenyl)pyrimidine (3r)

Yellow solid, $390.6 \mathrm{mg}$, yield: 76\%; m.p. 167.1-167.3 ${ }^{\circ} \mathrm{C}(\mathrm{EA} / \mathrm{PE})$; IR $\left(\mathrm{KBr}, \mathrm{cm}^{-1}\right): v$ 3435, 3057, 1580, 1529, 1482, 1370, 1074, 1005, 828, 782; ${ }^{1} \mathrm{H}$ NMR (400 MHz, $\left.\mathrm{CDCl}_{3}\right) \delta(\mathrm{ppm}): 8.44(\mathrm{~d}, J=7.6 \mathrm{~Hz}, 2 \mathrm{H}), 8,20(\mathrm{~d}, J=7.6 \mathrm{~Hz}, 2 \mathrm{H}), 7.98(\mathrm{~s}, 1 \mathrm{H}), 7.74$ $(\mathrm{d}, J=7.6 \mathrm{~Hz}, 2 \mathrm{H}), 7.56(\mathrm{~d}, J=7.6 \mathrm{~Hz}, 2 \mathrm{H}), 7.49$ (d, $J=7.2 \mathrm{~Hz}, 2 \mathrm{H}), 7.35$ (d, $J=7.6$ $\mathrm{Hz}, 2 \mathrm{H}), 4.15$ (s, 2H); ${ }^{13} \mathrm{C} \mathrm{NMR}\left(150 \mathrm{MHz}, \mathrm{CDCl}_{3}\right) \delta(p p m): 170.1,162.4,162.3$, 137.6, 135.9, 135.8, 135.3, 132.0(2C), 131.39(2C), 131.37(2C), 129.6(2C), 129.1(2C), 128.8(2C), 125.0, 119.8, 114.2, 42.7; HR-MS (ESI) calcd. for $\mathrm{C}_{23} \mathrm{H}_{16} \mathrm{Br}_{2} \mathrm{ClN}_{2}$ $\left[(\mathrm{M}+\mathrm{H})^{+}\right]:$514.9270; Found: 514.9266.

\section{2,4-bis(4-chlorophenyl)-6-(3-phenoxybenzyl)pyrimidine (3s)}

White solid, $250.7 \mathrm{mg}$, yield: 52\%; m.p. 116.9-117.1 ${ }^{\circ} \mathrm{C}(\mathrm{EA} / \mathrm{PE})$; IR $\left(\mathrm{KBr}, \mathrm{cm}^{-1}\right): v$ 3455, 3053, 1580, 1527, 1483, 1364, 1086, 1009, 833, 749; ${ }^{1} \mathrm{H}$ NMR (400 MHz, $\left.\mathrm{CDCl}_{3}\right) \delta(\mathrm{ppm}): 8.39(\mathrm{~d}, J=8.4 \mathrm{~Hz}, 2 \mathrm{H}), 8.24(\mathrm{~d}, J=8.4 \mathrm{~Hz}, 2 \mathrm{H}), 7.95(\mathrm{~s}, 1 \mathrm{H}), 7.58$ (d, $J=8.4 \mathrm{~Hz}, 2 \mathrm{H}), 7.53$ (d, $J=8.4 \mathrm{~Hz}, 2 \mathrm{H}), 7,33-7,28(\mathrm{~m}, 3 \mathrm{H}), 7.15$ (d, $J=7.2 \mathrm{~Hz}$, 1H), 7.10-7.07 (m, 2H), 6,96 (d, $J=7.6 \mathrm{~Hz}, 2 \mathrm{H}), 6.83(\mathrm{~d}, J=6.8 \mathrm{~Hz}, 1 \mathrm{H}), 4.14$ (s, $1 \mathrm{H}) ;{ }^{13} \mathrm{C} \mathrm{NMR}\left(150 \mathrm{MHz}, \mathrm{CDCl}_{3}\right) \delta(\mathrm{ppm}): 170.2,162.2,162.1,156.8,156.5,140.3$, $136.1,135.9,135.8,134.9,130.1,129.9(2 \mathrm{C}), 129.5(2 \mathrm{C}), 129.0(2 \mathrm{C}), 128.8(2 \mathrm{C})$, 128.7(2C), 124.2, 123.5, 119.2, 118.7(2C), 116.7, 114.2, 43.1; HR-MS (ESI) calcd. for $\mathrm{C}_{29} \mathrm{H}_{21} \mathrm{Cl}_{2} \mathrm{~N}_{2} \mathrm{O}\left[(\mathrm{M}+\mathrm{H})^{+}\right]$: 483.1025; Found: 483.1071

\section{4-(2-bromobenzyl)-2,6-bis(4-bromophenyl)pyrimidine (3t)}

White solid, $429.6 \mathrm{mg}$, yield: 77\%; m.p. 168.3-168.5 ${ }^{\circ} \mathrm{C}(\mathrm{EA} / \mathrm{PE})$; IR $\left(\mathrm{KBr}, \mathrm{cm}^{-1}\right): v$ 
3724, 3059, 1578, 1529, 1481, 1371, 1069, 1011, 830, 786; ${ }^{1} \mathrm{H}$ NMR (400 MHz, $\left.\mathrm{CDCl}_{3}\right) \delta(p p m): 8.34(\mathrm{~d}, J=8.4 \mathrm{~Hz}, 2 \mathrm{H}), 8.2(\mathrm{~d}, J=8.4 \mathrm{~Hz}, 2 \mathrm{H}), 7.9(\mathrm{~s}, 1 \mathrm{H}), 7.77(\mathrm{~d}$, $J=8.4 \mathrm{~Hz}, 2 \mathrm{H}), 7.71(\mathrm{~d}, J=8.4 \mathrm{~Hz}, 2 \mathrm{H}), 7,64(\mathrm{~d}, J=8.0 \mathrm{~Hz}, 1 \mathrm{H}), 7.48(\mathrm{~d}, J=7.6 \mathrm{~Hz}$, 1H), $7.37(\mathrm{dd}, J=7.2,7.6 \mathrm{~Hz} 1 \mathrm{H}), 7.22(\mathrm{dd}, J=7.6,8.0 \mathrm{~Hz} 1 \mathrm{H}), 4.35(\mathrm{~s}, 2 \mathrm{H}) ;{ }^{13} \mathrm{C}$ NMR $\left(150 \mathrm{MHz}, \mathrm{CDCl}_{3}\right) \delta(p p m): 169.4,162.4,137.3,136.3,135.3,132.6$, 132.08(2C), 132.06(2C), 131.7, 129.8(2C), 129.1(2C), 128.9, 127.9, 125.1, 124.8, 124.3, 114.3, 104.5, 43.4; HR-MS (ESI) calcd. for $\mathrm{C}_{23} \mathrm{H}_{16} \mathrm{Br}_{3} \mathrm{~N}_{2}\left[(\mathrm{M}+\mathrm{H})^{+}\right]$: 558.8838; Found: 558.8853.

\section{4-(4-bromobenzyl)-2-(4-bromophenyl)-6-(4-chlorophenyl)pyrimidine (3u)}

White solid, $400.8 \mathrm{mg}$, yield: $78 \%$; m.p. $169.4-169.6{ }^{\circ} \mathrm{C}(\mathrm{EA} / \mathrm{PE})$; IR $\left(\mathrm{KBr}, \mathrm{cm}^{-1}\right): v$ 3450, 3062, 1580, 1530, 1483, 1371, 1075, 1006, 830, 782; ${ }^{1} \mathrm{H}$ NMR (400 MHz, $\left.\mathrm{CDCl}_{3}\right) \delta(p p m): 8.38(\mathrm{~d}, J=7.6 \mathrm{~Hz}, 2 \mathrm{H}), 8.30(\mathrm{~d}, J=8.0 \mathrm{~Hz}, 2 \mathrm{H}), 8.01(\mathrm{~s}, 1 \mathrm{H}), 7.72$ $(\mathrm{d}, J=8.4 \mathrm{~Hz}, 2 \mathrm{H}), 7.62(\mathrm{~d}, J=8.0 \mathrm{~Hz}, 2 \mathrm{H}), 7.49$ (d, $J=8.0 \mathrm{~Hz}, 2 \mathrm{H}), 7.36(\mathrm{~d}, J=8.0$ $\mathrm{Hz}, 2 \mathrm{H}), 4.16(\mathrm{~s}, 2 \mathrm{H}) ;{ }^{13} \mathrm{C} \mathrm{NMR}\left(150 \mathrm{MHz}, \mathrm{CDCl}_{3}\right) \delta(p p m): 170.1,162.4,162.3$, 137.6, 136.3, 135.3, 132.0(2C), 131.7(2C), 131.4(2C), 131.3, 129.8(2C), 129.1(2C), 128.9(2C), 124.8, 119.8, 114.3, 42,7; HR-MS (ESI) calcd. for $\mathrm{C}_{23} \mathrm{H}_{16} \mathrm{Br}_{2} \mathrm{ClN}_{2}$ $\left[(\mathrm{M}+\mathrm{H})^{+}\right]:$514.9343; Found: 514.9356 .

\section{2,4-bis(4-bromophenyl)-6-(4-chlorobenzyl)pyrimidine (3v)}

White solid, $292.9 \mathrm{mg}$, yield: 57\%; m.p. $179.3-179.5{ }^{\circ} \mathrm{C}(\mathrm{EA} / \mathrm{PE})$; IR $\left(\mathrm{KBr}, \mathrm{cm}^{-1}\right): v$ 3455, 2990, 1581, 1529, 1485, 1370, 1175, 1080, 830, 783; ${ }^{1} \mathrm{H}$ NMR (400 MHz, $\left.\mathrm{CDCl}_{3}\right) \delta(\mathrm{ppm}): 8.39(\mathrm{~d}, J=8.4 \mathrm{~Hz}, 2 \mathrm{H}), 8.23(\mathrm{~d}, J=8.4 \mathrm{~Hz}, 2 \mathrm{H}), 8.02(\mathrm{~s}, 1 \mathrm{H}), 7.77$ $(\mathrm{d}, J=8.4 \mathrm{~Hz} 2 \mathrm{H}), 7.73(\mathrm{~d}, J=8.4 \mathrm{~Hz} 2 \mathrm{H}), 7.43(\mathrm{~d}, J=8.4 \mathrm{~Hz}, 2 \mathrm{H}), 7.36(\mathrm{~d}, J=8.4$ $\mathrm{Hz}, 2 \mathrm{H}), 4.18$ (s, 2H); ${ }^{13} \mathrm{C} \mathrm{NMR}\left(150 \mathrm{MHz}, \mathrm{CDCl}_{3}\right) \delta(p p m): 170.3,162.4,162.3$, 137.2, 136.3, 135.3, 132.0, 131.7(2C), 131.3(2C), 130.9(2C), 129.8(2C), 129.1(2C), 128.5(2C), 125.1, 124.8, 114.3, 42.6; HR-MS (ESI) calcd. for $\mathrm{C}_{23} \mathrm{H}_{16} \mathrm{Br}_{2} \mathrm{ClN}_{2}$ $\left[(\mathrm{M}+\mathrm{H})^{+}\right]:$514.9343; Found: 514.9340 .

2-(4-bromophenyl)-4-(4-chlorophenyl)-6-(3-phenoxybenzyl)pyrimidine (3w) 
White solid, $426.1 \mathrm{mg}$, yield: 81\%; m.p. 132.6-132.8 ${ }^{\circ} \mathrm{C}(\mathrm{EA} / \mathrm{PE})$; IR $\left(\mathrm{KBr}, \mathrm{cm}^{-1}\right): v$ 3455, 3050, 1580, 1535, 1480, 1365, 1087, 1010, 835, 750; ${ }^{1} \mathrm{H}$ NMR (400 MHz, $\left.\mathrm{CDCl}_{3}\right) \delta(\mathrm{ppm}): 8.36(\mathrm{~d}, J=7.2 \mathrm{~Hz}, 2 \mathrm{H}), 8.29(\mathrm{~d}, J=7.6 \mathrm{~Hz}, 2 \mathrm{H}), 8.02(\mathrm{~s}, 1 \mathrm{H}), 7.72$ $(\mathrm{d}, J=7.6 \mathrm{~Hz}, 2 \mathrm{H}), 7.63(\mathrm{~d}, J=7.2 \mathrm{~Hz}, 2 \mathrm{H}), 7.34-7,29$ (m, 3H), 7.16 (d, $J=7.2 \mathrm{~Hz}$, 1H), 7.12-7.09 (m, 2H), 6.97 (d, $J=7.6 \mathrm{~Hz}, 2 \mathrm{H}), 6.84(\mathrm{~d}, J=8.0,1 \mathrm{H}), 4.17(\mathrm{~s}, 2 \mathrm{H})$; ${ }^{13} \mathrm{C}$ NMR $\left(150 \mathrm{MHz}, \mathrm{CDCl}_{3}\right) \delta(p p m): 170.3,162.3,162.2,156.8,156.5,140.3,136.3$, $136.1,134.9,131.7(2 \mathrm{C}), 130.1(2 \mathrm{C}), 129.9(2 \mathrm{C}), 129.8(2 \mathrm{C}), 129.1(2 \mathrm{C}), 128.9(2 \mathrm{C})$, 124.8, 124.2, 123.4, 119.2, 118.7(2C), 116.7, 114.3, 43.1; HR-MS (ESI) calcd. for $\mathrm{C}_{29} \mathrm{H}_{21} \mathrm{BrClN}_{2} \mathrm{O}\left[(\mathrm{M}+\mathrm{H})^{+}\right]$: 527.0520; Found: 527.0527.

4-(4-bromophenyl)-6-(4-chlorobenzyl)-2-(p-tolyl)pyrimidine (3x)

White solid, $327.1 \mathrm{mg}$, yield: $73 \%$; m.p. $132.4-132.5{ }^{\circ} \mathrm{C}(\mathrm{EA} / \mathrm{PE})$; IR $\left(\mathrm{KBr}, \mathrm{cm}^{-1}\right): v$ 3565, 2980, 1540, 1517, 1479, 1383, 1169, 1075, 825, 779; ${ }^{1} \mathrm{H}$ NMR (400 MHz, $\left.\mathrm{CDCl}_{3}\right) \delta($ ppm): $8.36(\mathrm{~d}, J=8 \mathrm{~Hz}, 2 \mathrm{H}), 8.30(\mathrm{~d}, J=8 \mathrm{~Hz}, 2 \mathrm{H}), 7.94$ (s, 1H), 7.62 (d, $J=8.4 \mathrm{~Hz}, 2 \mathrm{H}), 7.50(\mathrm{~d}, J=9.2 \mathrm{~Hz}, 2 \mathrm{H}), 7.36(\mathrm{~d}, J=8.4 \mathrm{~Hz}, 2 \mathrm{H}), 7.32(\mathrm{~d}, J=7.6 \mathrm{~Hz}, 2 \mathrm{H})$, 4.15 (s, 2H), 2.36 (s, 3H); ${ }^{13} \mathrm{C}$ NMR (150 MHz, $\left.\mathrm{CDCl}_{3}\right) \delta(p p m): 170.4,163.8,162.5$, 141.2, 138.2, 136.4(2C), 135.6(2C), 134.9(2C), 131.8(3C), 129.7, 129.5, 129.3(2C), 128.3(2C), 120.2, 114.2, 43.2, 21.5; HR-MS (ESI) calcd. for $\mathrm{C}_{24} \mathrm{H}_{19} \mathrm{BrClN}_{2}\left[(\mathrm{M}+\mathrm{H})^{+}\right]$: 449.0420; Found: 449.0415.

4-(4-chlorobenzyl)-6-(4-chlorophenyl)-2-(p-tolyl)pyrimidine (3y)

White solid, $279 \mathrm{mg}$, yield: 69\%; m.p. 105.4-105.6 ${ }^{\circ} \mathrm{C}$ (EA/PE); IR (KBr, $\left.\mathrm{cm}^{-1}\right): v$ 3485, 2881, 1632, 1545, 1463, 1387, 1152, 1067, 813, 789; ${ }^{1} \mathrm{H}$ NMR (400 MHz, $\left.\mathrm{CDCl}_{3}\right) \delta(\mathrm{ppm}): 8.36(\mathrm{~d}, J=6.8 \mathrm{~Hz}, 2 \mathrm{H}), 8.30(\mathrm{~d}, J=8 \mathrm{~Hz}, 2 \mathrm{H}), 7.94(\mathrm{~s}, 1 \mathrm{H}), 7.62$ (d, $J=8 \mathrm{~Hz}, 2 \mathrm{H}), 7.43$ (d, $J=7.6 \mathrm{~Hz}, 2 \mathrm{H}), 7.36$ (d, $J=7.6 \mathrm{~Hz}, 2 \mathrm{H}), 7.32$ (d, $J=8 \mathrm{~Hz}, 2 \mathrm{H})$, $4.17(\mathrm{~s}, 2 \mathrm{H}), 2.36(\mathrm{~s}, 3 \mathrm{H}) ;{ }^{13} \mathrm{C} \mathrm{NMR}\left(600 \mathrm{MHz}, \mathrm{CDCl}_{3}\right) \delta(\mathrm{ppm}): 170.4,163.8,162.5$, 141.2, 137.8(2C), 136.4(2C), 135.6(2C), 134.9(2C), 131.7, 131.4, 129.8, 129.7, 129.5, 129.3(2C), 128.9, 128.3(2C), 43.1, 21.5; HR-MS (ESI) calcd. for $\mathrm{C}_{24} \mathrm{H}_{19} \mathrm{Cl}_{2} \mathrm{~N}_{2}$ $\left[(\mathrm{M}+\mathrm{H})^{+}\right]:$405.0925; Found: 405.0924. 
4-(4-Bromophenyl)-2-methyl-6-(4-methylbenzyl)pyrimidine (3z)

Oil, $207.1 \mathrm{mg}$, yield: $59 \%$; IR $\left(\mathrm{KBr}, \mathrm{cm}^{-1}\right): v 3340,2991,1568,1528,1420,1360$, 1170, 1086, 836, 880; ${ }^{1} \mathrm{H} \mathrm{NMR}\left(400 \mathrm{MHz}, \mathrm{CDCl}_{3}\right) \delta(p p m): 7.87(\mathrm{~d}, J=7.6 \mathrm{~Hz}, 2 \mathrm{H})$, $7.44(\mathrm{~d}, J=6.8 \mathrm{~Hz}, 2 \mathrm{H}), 7.25$ (d, $J=6.4 \mathrm{~Hz} 2 \mathrm{H}), 7.20$ (s, 1H), 7.17 (d, $J=7.2 \mathrm{~Hz} 2 \mathrm{H})$, $4.06(\mathrm{~s}, 2 \mathrm{H}), 2.77(\mathrm{~s}, 3 \mathrm{H}), 2.39(\mathrm{~s}, 3 \mathrm{H}) ;{ }^{13} \mathrm{C} \mathrm{NMR}\left(100 \mathrm{MHz}, \mathrm{CDCl}_{3}\right) \delta(\mathrm{ppm}): 168.7$, 168.1, 164.5, 141.1, 136.7, 134.1(2C), 131.9(2C), 130.9(2C), 128.6, 127.1(2C), 120.8, 112.6, 43.6, 26,2, 21.4; HR-MS (ESI) calcd. for $\mathrm{C}_{19} \mathrm{H}_{17} \mathrm{BrN}_{2}\left[(\mathrm{M}+\mathrm{H})^{+}\right]$: 352.0575; Found: 352.0571.

4-(4-Bromobenzyl)-6-(4-chlorophenyl)-2-methylpyrimidine (3aa)

Oil, $215.2 \mathrm{mg}$, yield: 58 \%; IR (KBr, cm $\left.{ }^{-1}\right): v 3390,2990,1572,1520,1422,1366$, 1180, 1076, 902, 877; ${ }^{1} \mathrm{H}$ NMR (400 MHz, $\left.\mathrm{CDCl}_{3}\right) \delta(p p m): 7.85(\mathrm{~d}, J=9.2 \mathrm{~Hz}, 2 \mathrm{H})$, 7.57 (d, $J=8.0 \mathrm{~Hz}, 2 \mathrm{H}), 7.29$ (d, $J=6.8 \mathrm{~Hz} 2 \mathrm{H}), 7.21$ (d, $J=7.6 \mathrm{~Hz} 2 \mathrm{H}), 7.19$ (s, 1H), $4.08(\mathrm{~s}, 2 \mathrm{H}), 2.77(\mathrm{~s}, 3 \mathrm{H}) ;{ }^{13} \mathrm{C} \mathrm{NMR}\left(100 \mathrm{MHz}, \mathrm{CDCl}_{3}\right) \delta($ ppm $): 169.4,168.3,163.3$, 136.1, 135.9, 132.8, 132.0(2C), 130.6(2C), 128.9(2C), 128.7(2C), 125.4, 112.6, 43.6, 26.2; HR-MS (ESI) calcd. for $\mathrm{C}_{18} \mathrm{H}_{14} \mathrm{BrClN}_{2}\left[(\mathrm{M}+\mathrm{H})^{+}\right]$: 372.0029 ; Found: 372.0027. 
Ethyl 2-(4-bromobenzoyl)-3-(4-chlorophenyl)-1-cyanocyclopropane-1-carboxylate (1b)
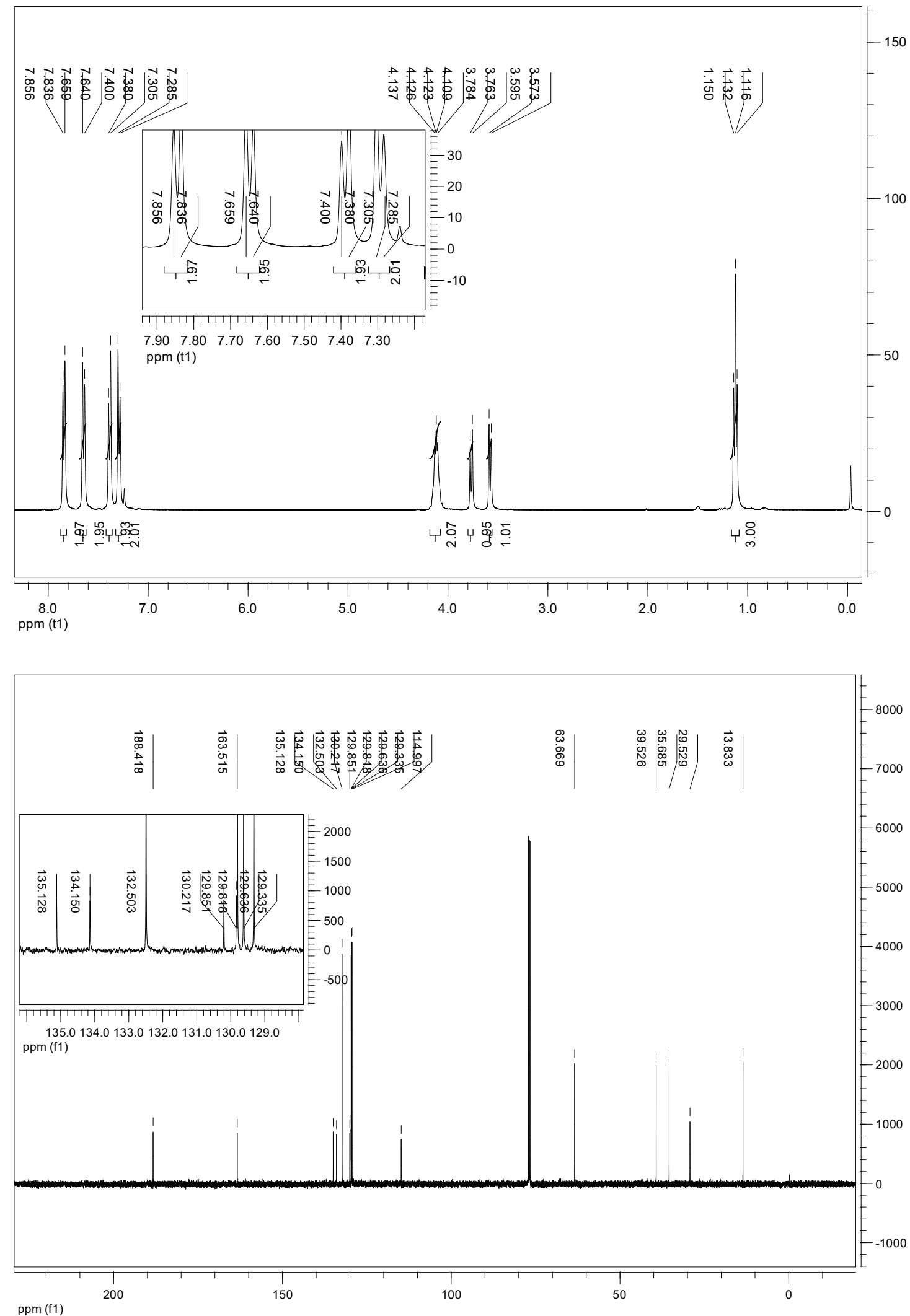
Ethyl 2-(2-bromophenyl)-1-cyano-3-(4-methoxybenzoyl)cyclopropane-1-carboxylate (1i)
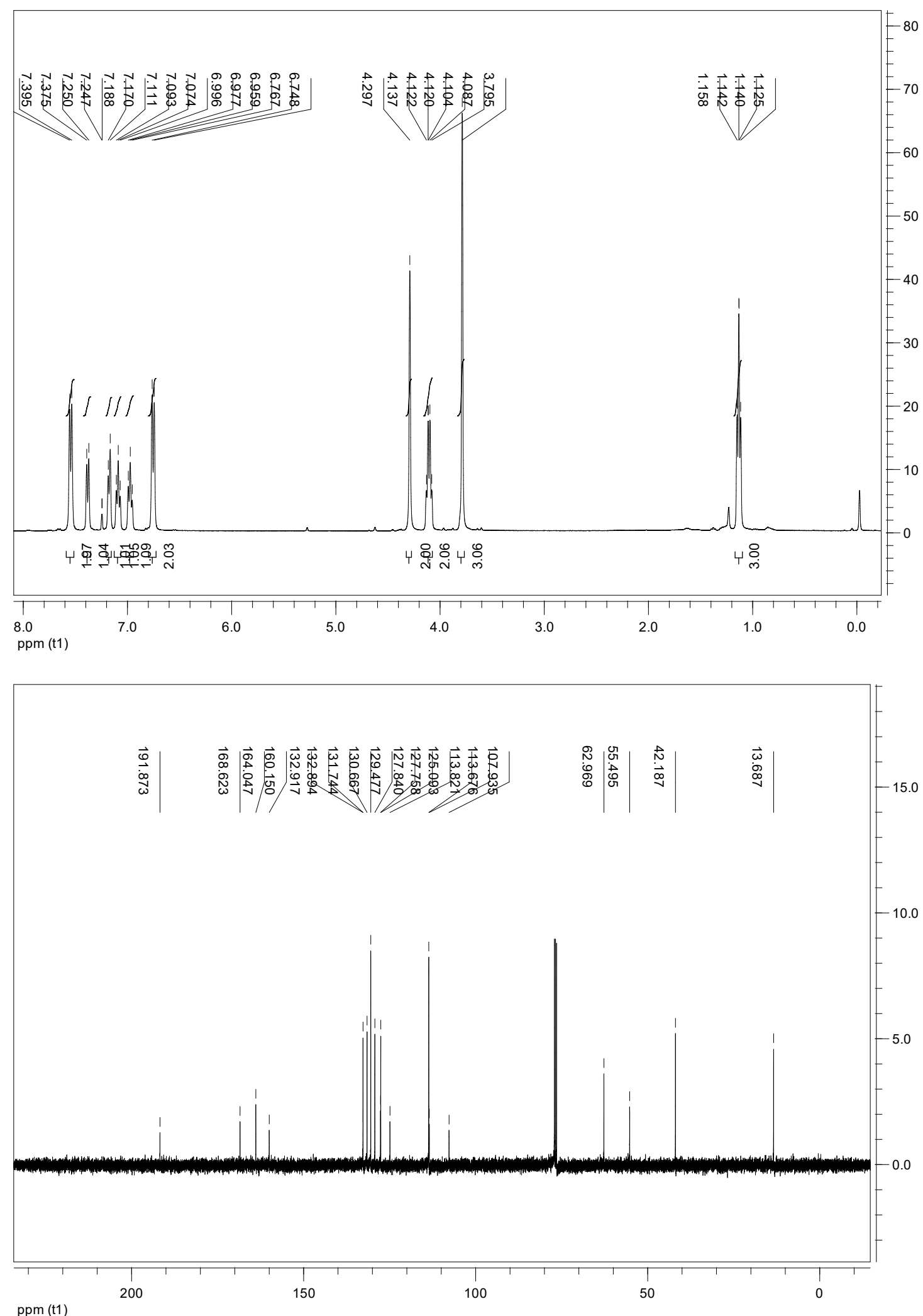
Ethyl 2-(4-chlorophenyl)-1-cyano-3-(4-methylbenzoyl)cyclopropane-1-carboxylate

(1j)
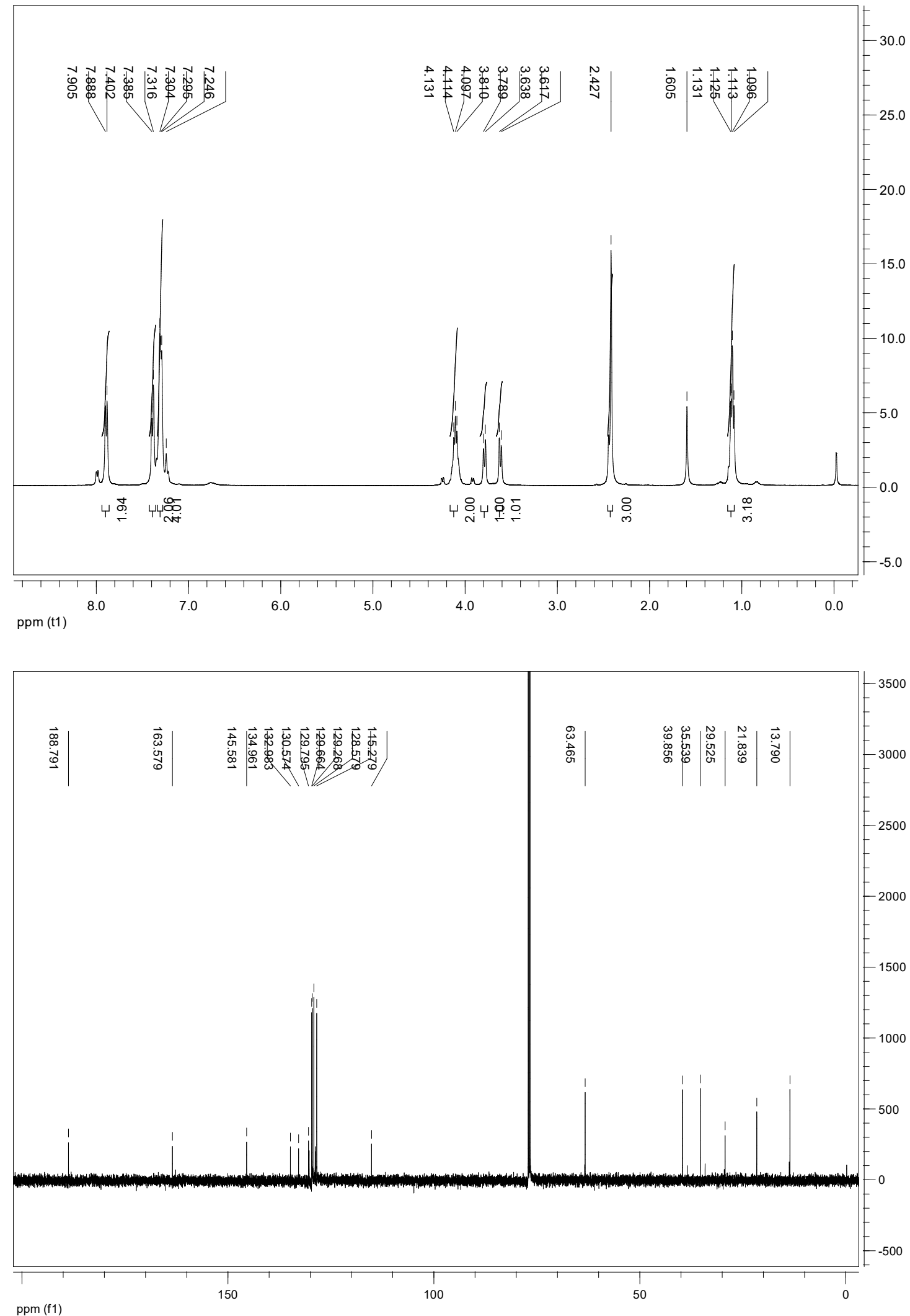
Ethyl 2-(4-chlorobenzoyl)-1-cyano-3-(thiophen-3-yl)cyclopropane-1-carboxylate (1n)

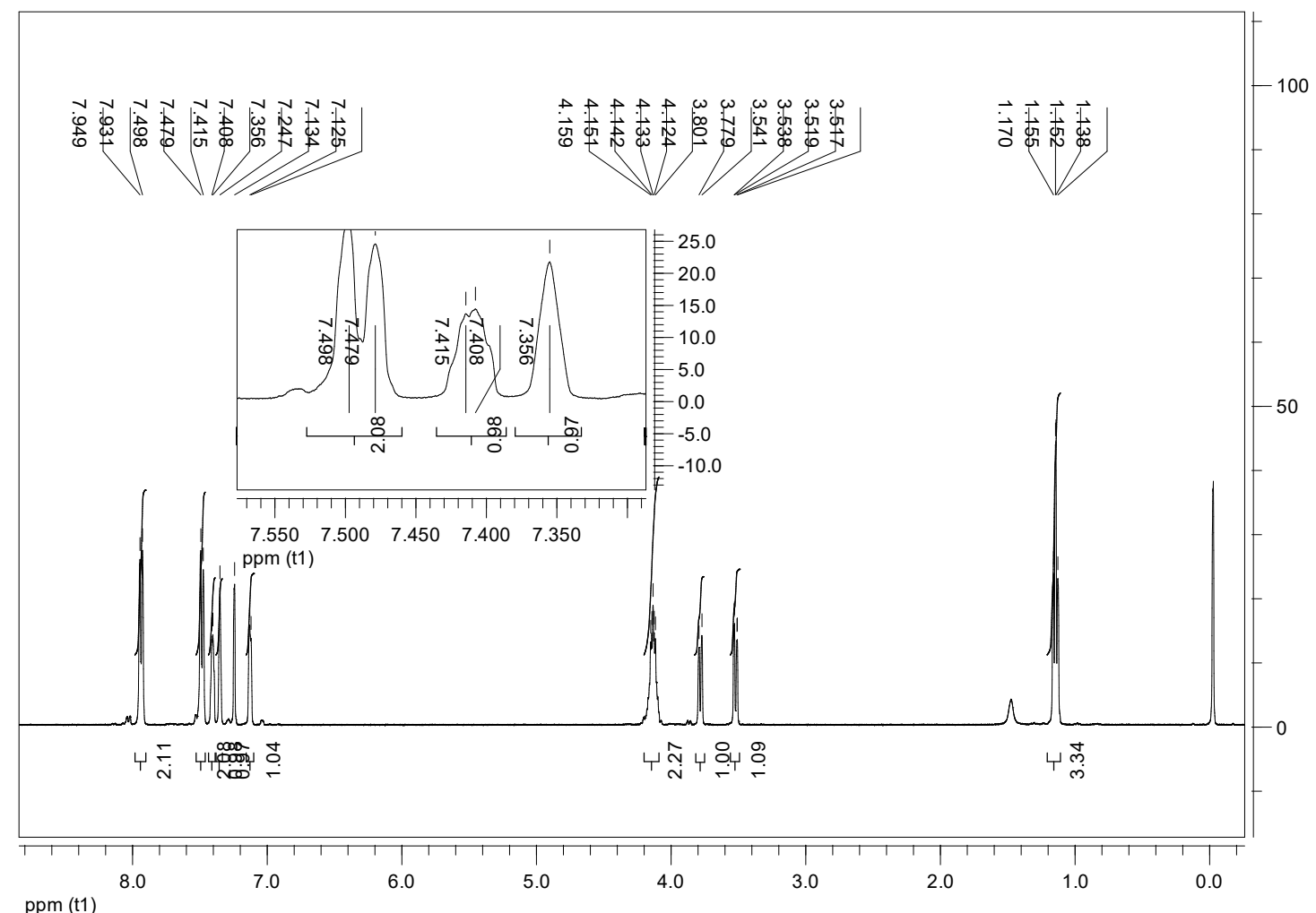

ppm (t1)

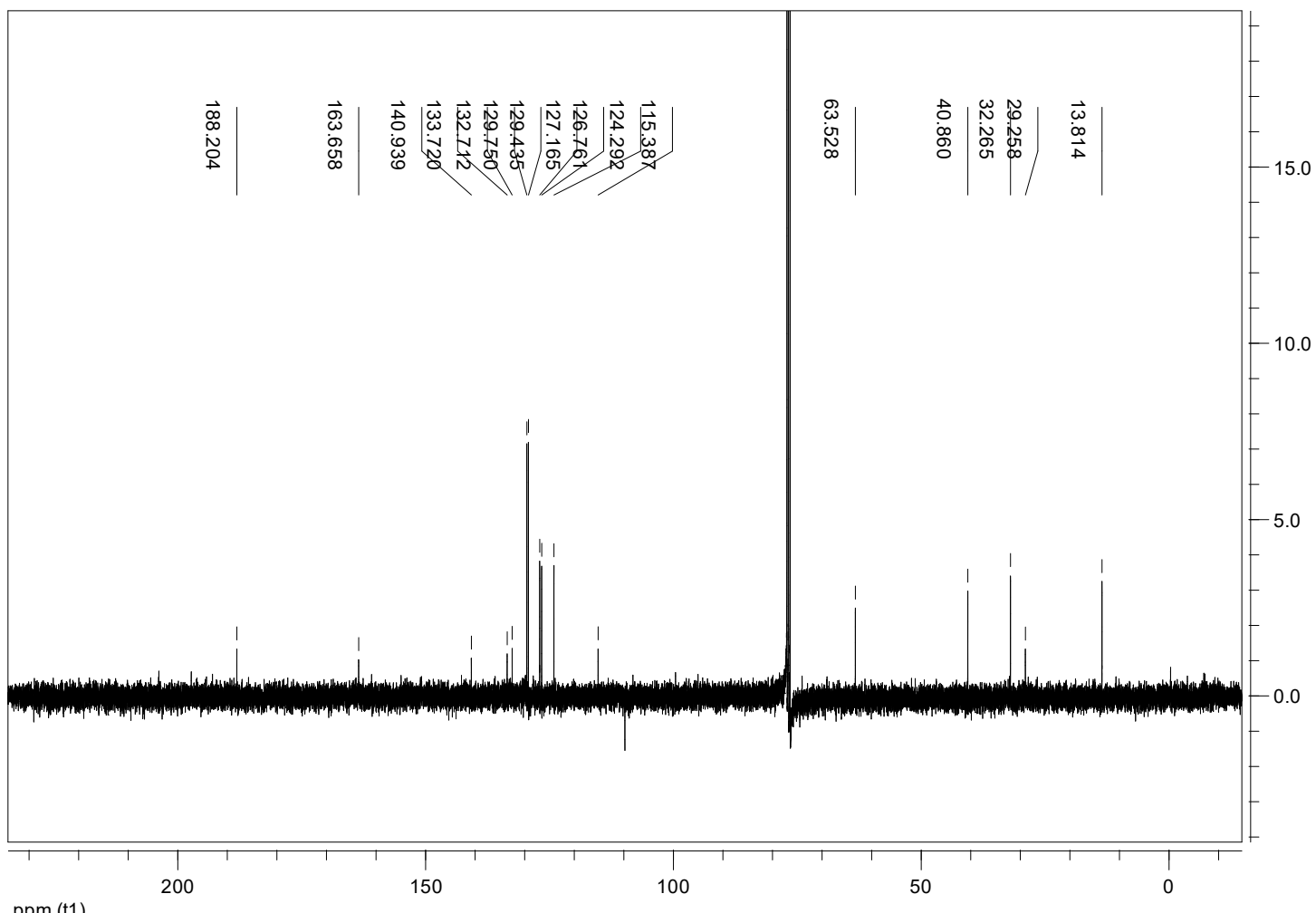


4-(2-chlorobenzyl)-2-phenyl-6-(p-tolyl)pyrimidine (3a)

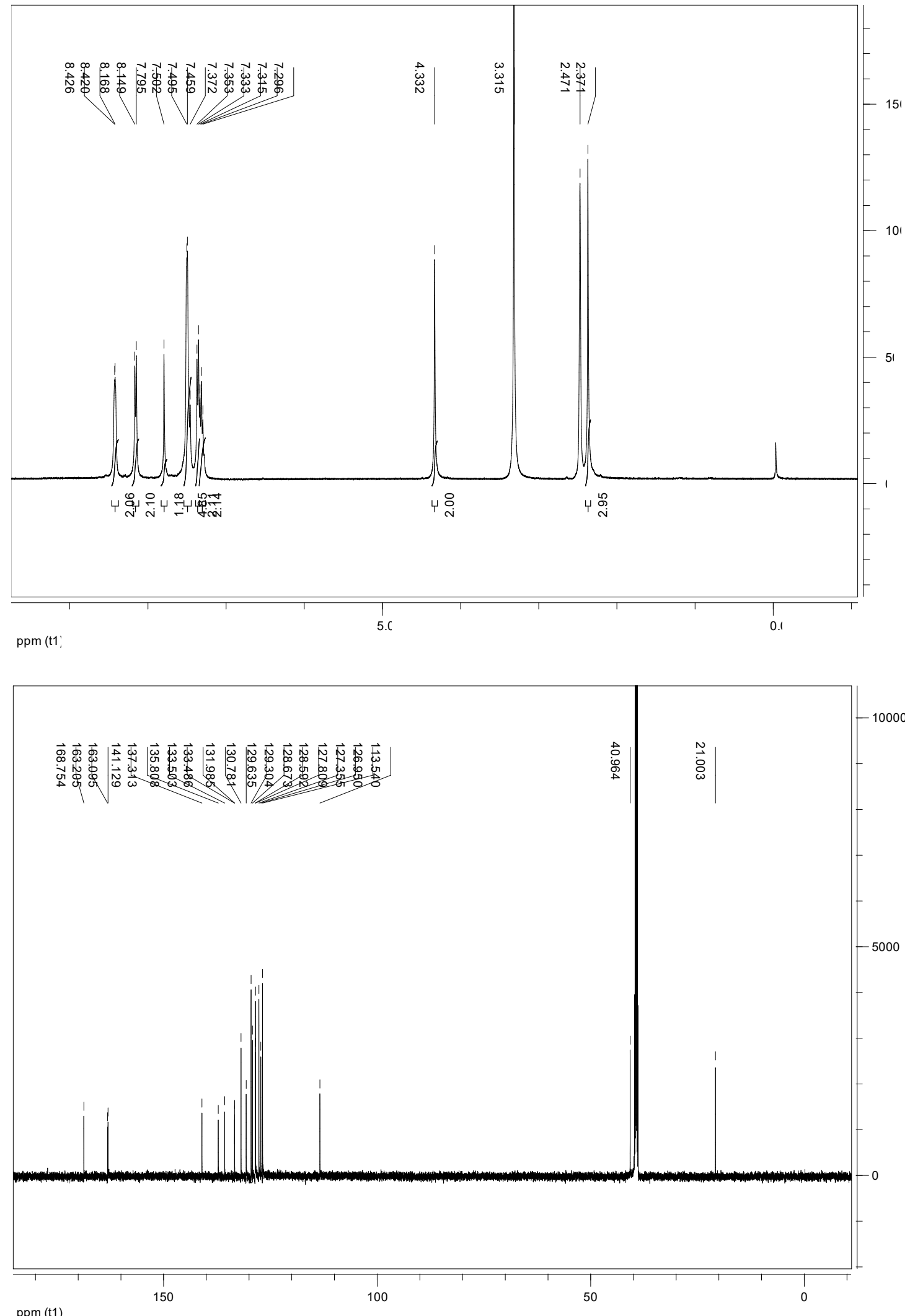


4-(4-bromophenyl)-6-(4-chlorobenzyl)-2-phenylpyrimidine (3b)

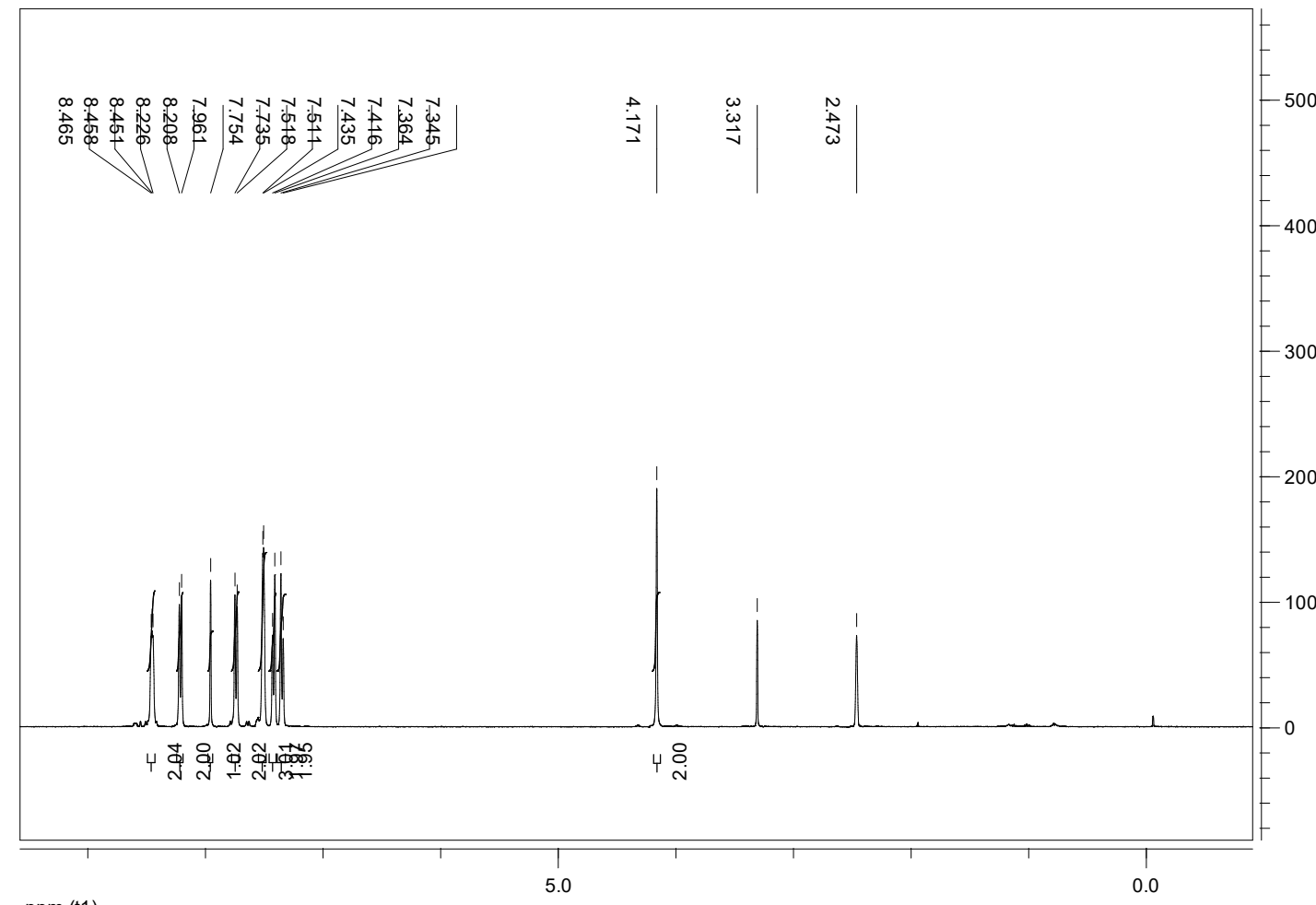

ppm (t1)

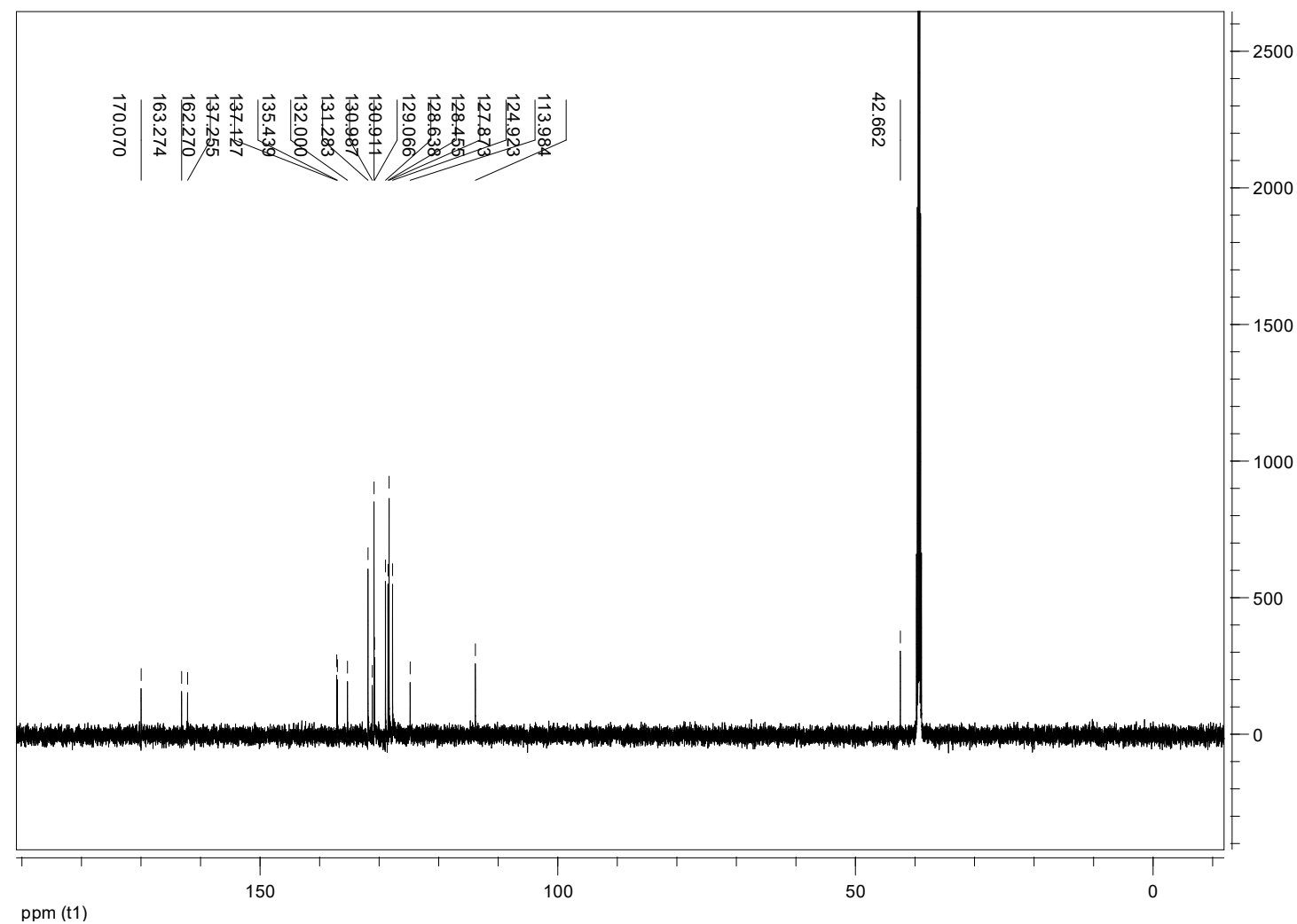


4-(2-chlorobenzyl)-6-(4-chlorophenyl)-2-phenylpyrimidine (3c)
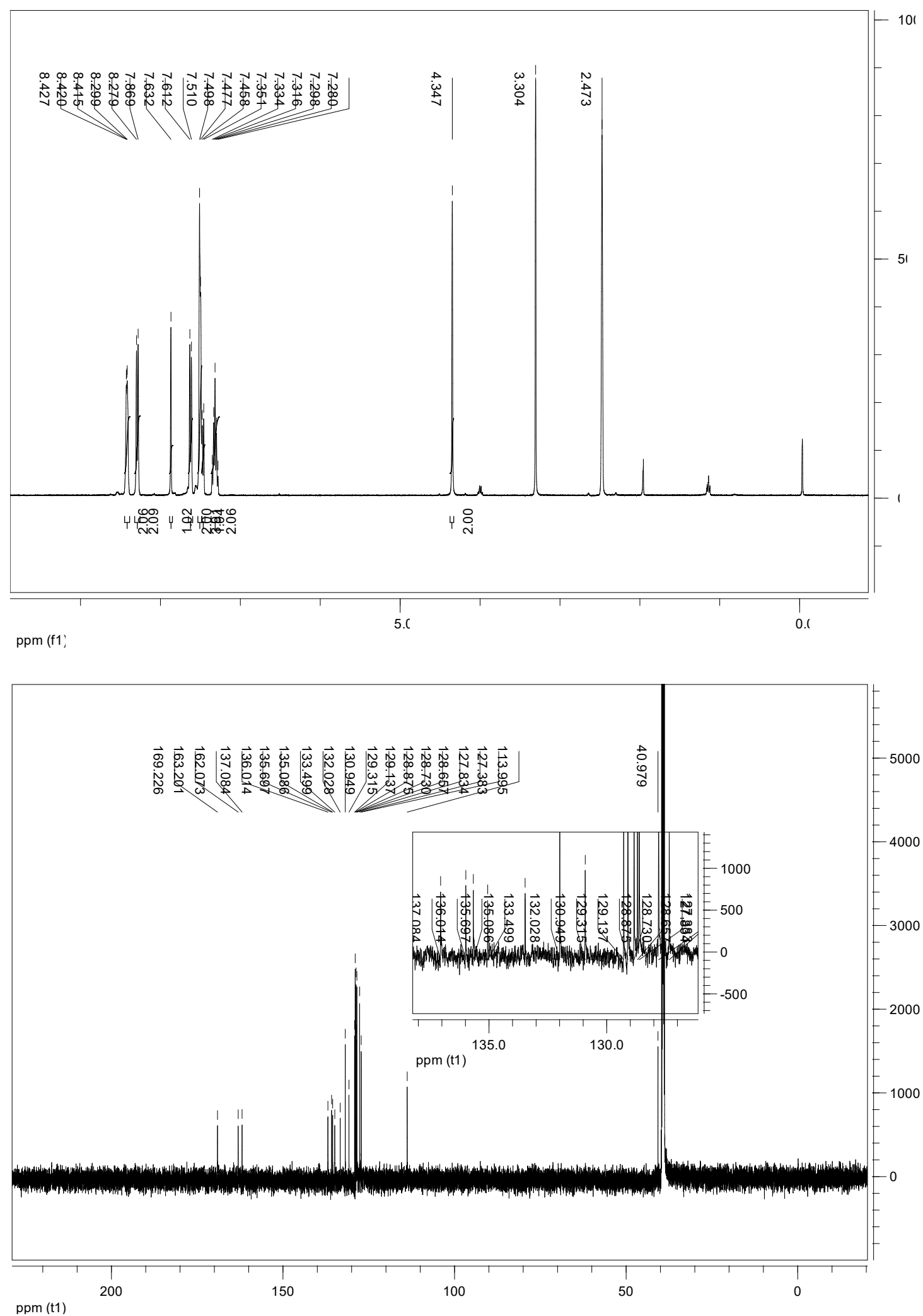
4-(2-bromobenzyl)-6-(4-bromophenyl)-2-phenylpyrimidine (3d)

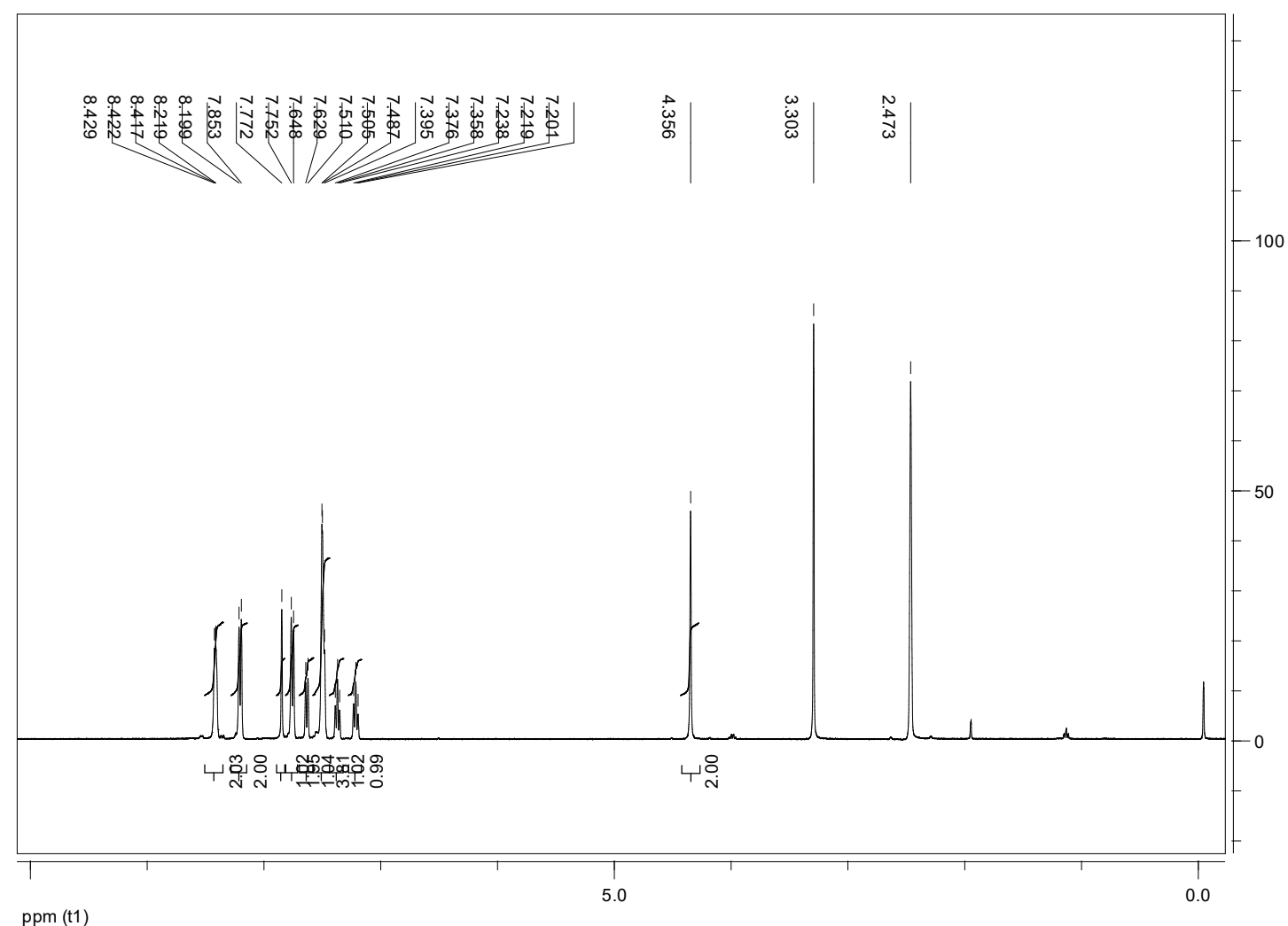

ppm (t1)

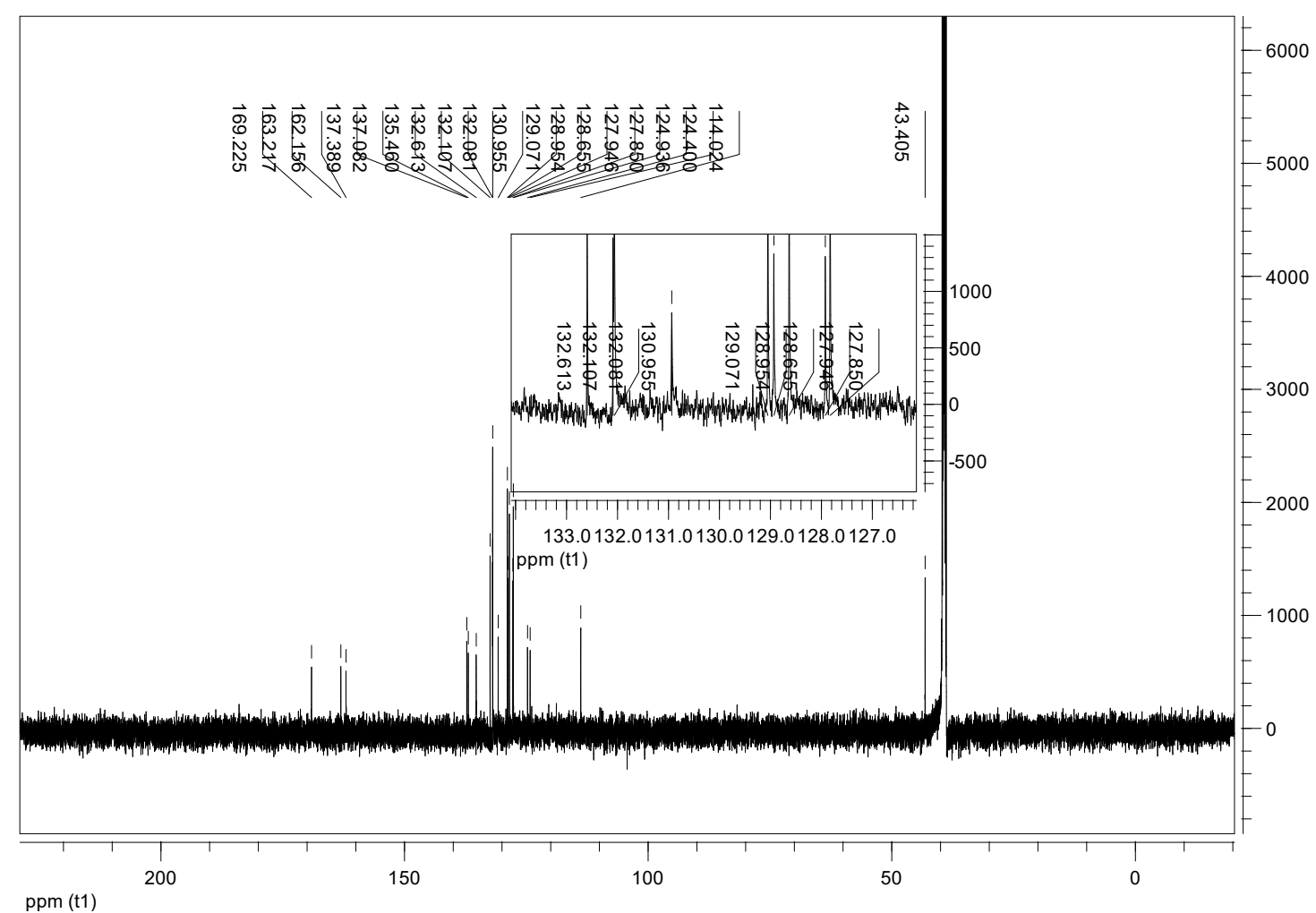




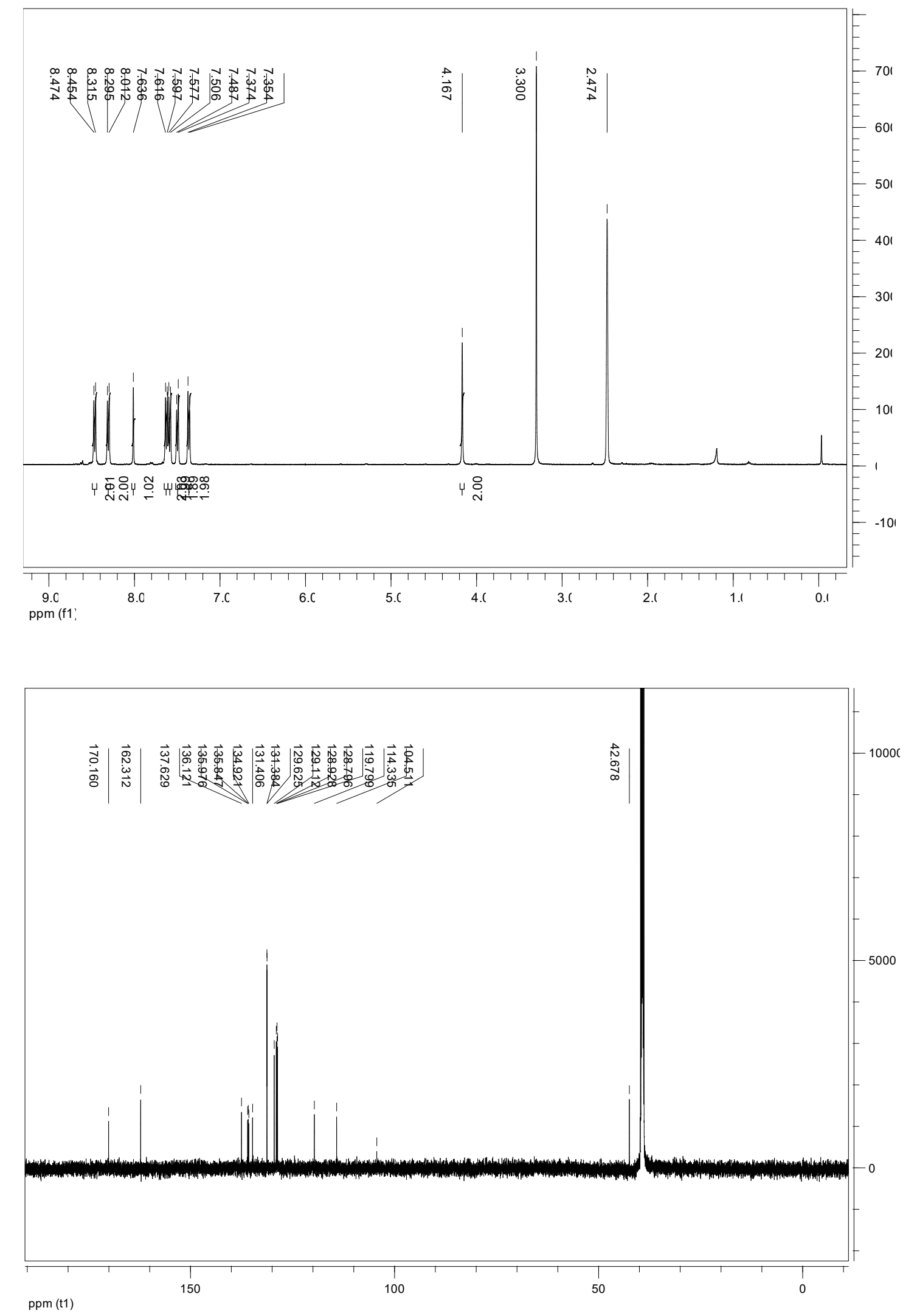


4-(4-bromophenyl)-6-(4-chlorobenzyl)-2-(4-chlorophenyl)pyrimidine (3f)
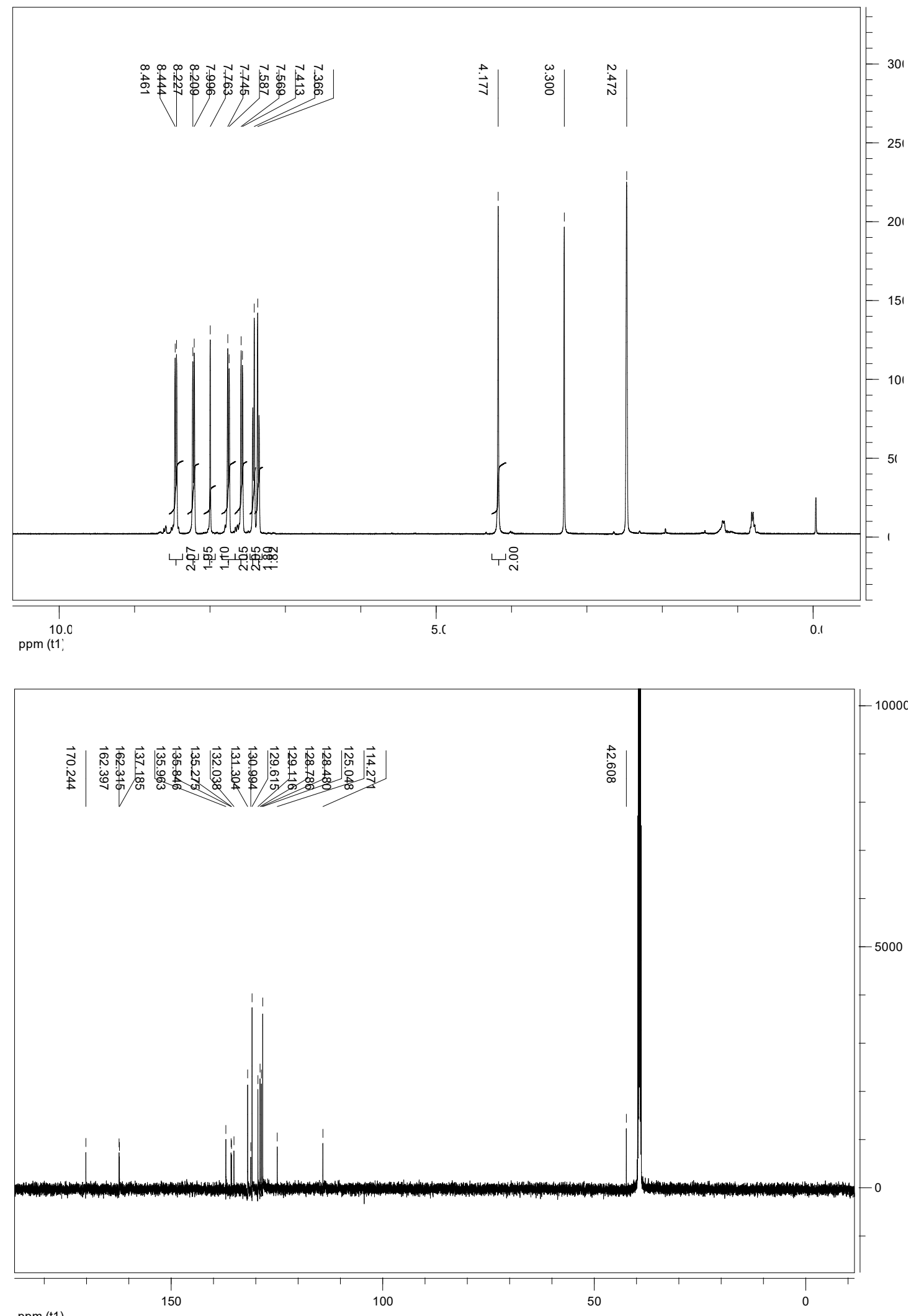
4-(2-bromobenzyl)-2,6-bis(4-chlorophenyl)pyrimidine (3g)
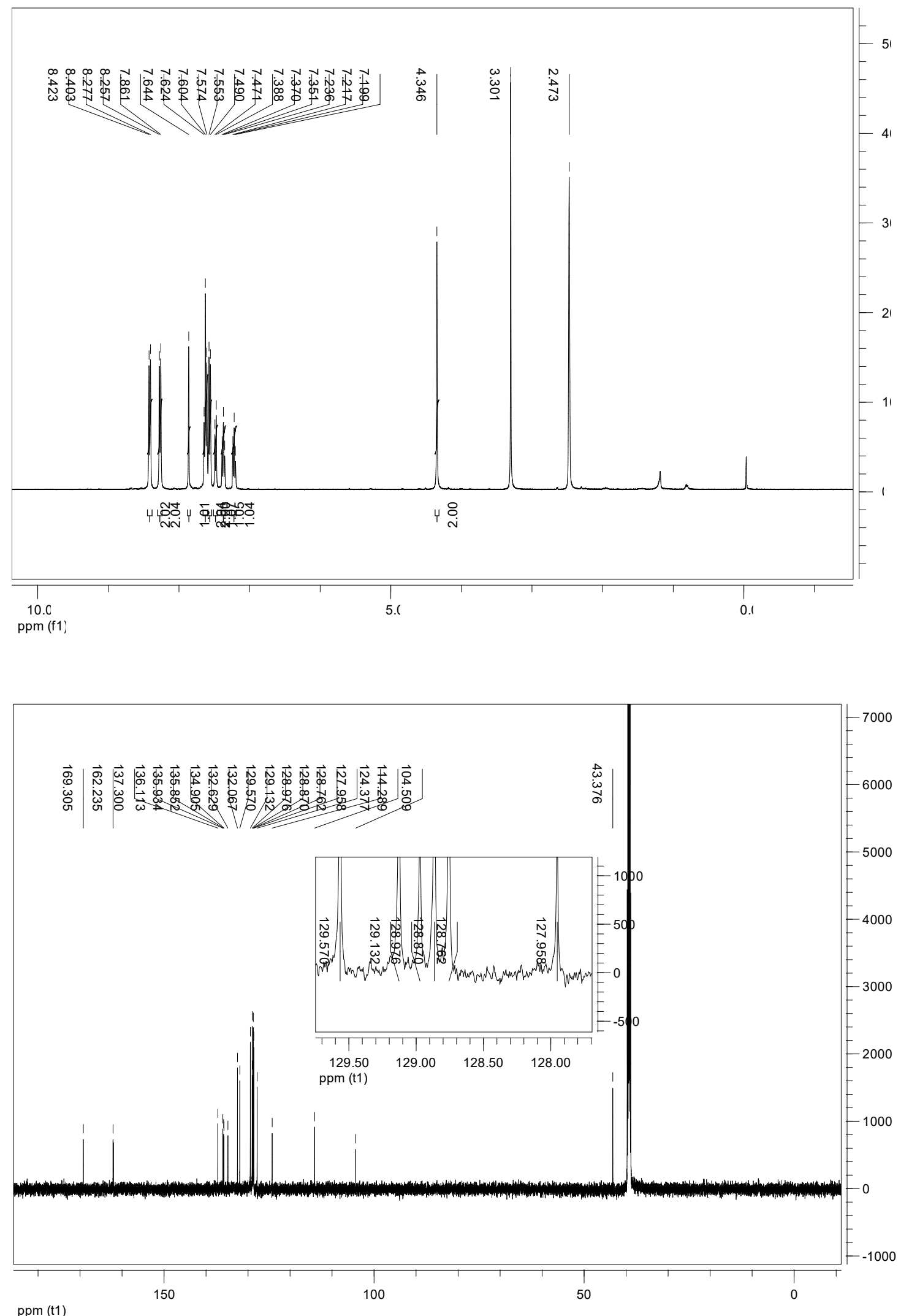
4-(3-chlorobenzyl)-2,6-bis(4-chlorophenyl)pyrimidine (3h)

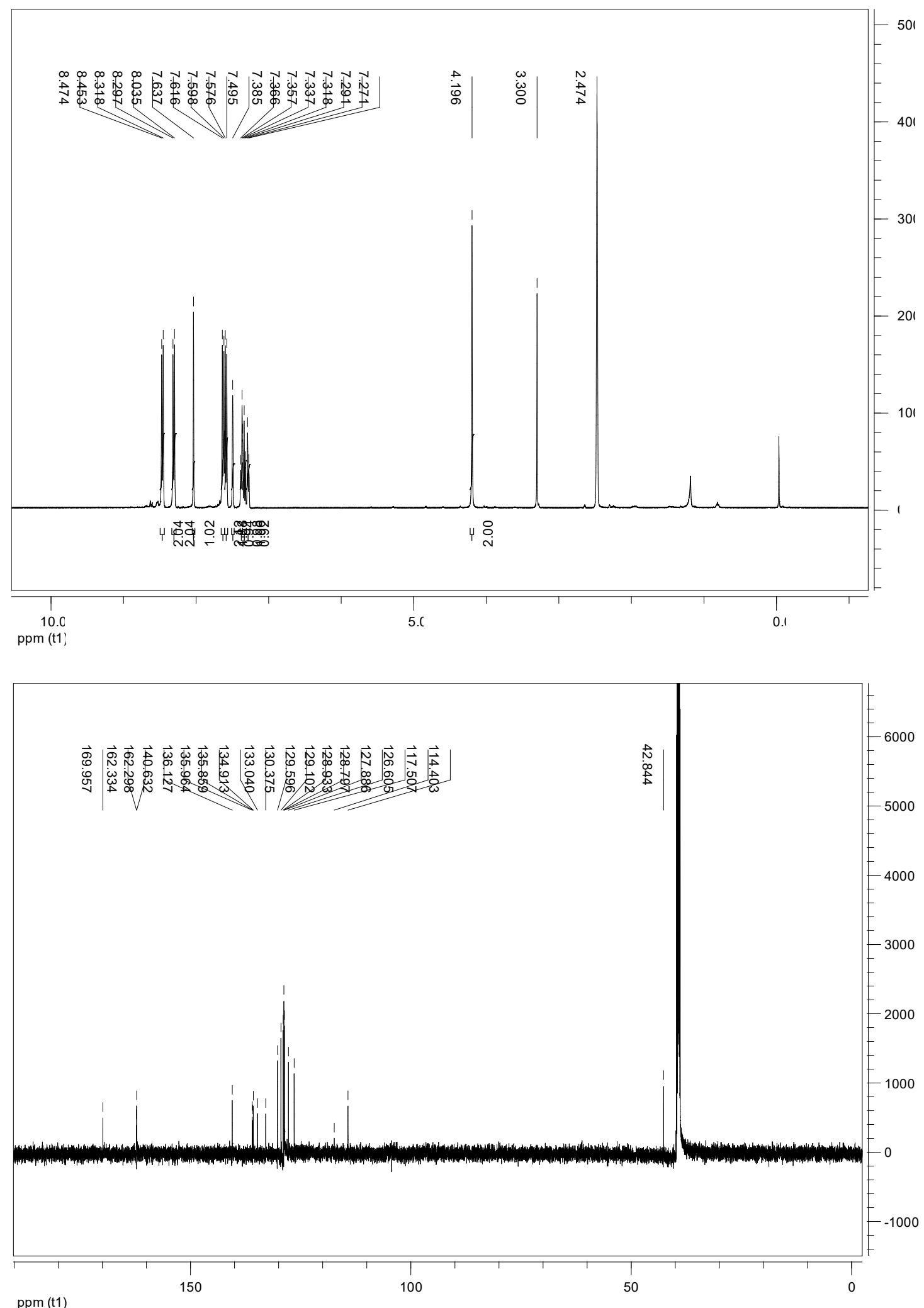


4-(3-chlorobenzyl)-2-(4-chlorophenyl)-6-phenylpyrimidine (3i)

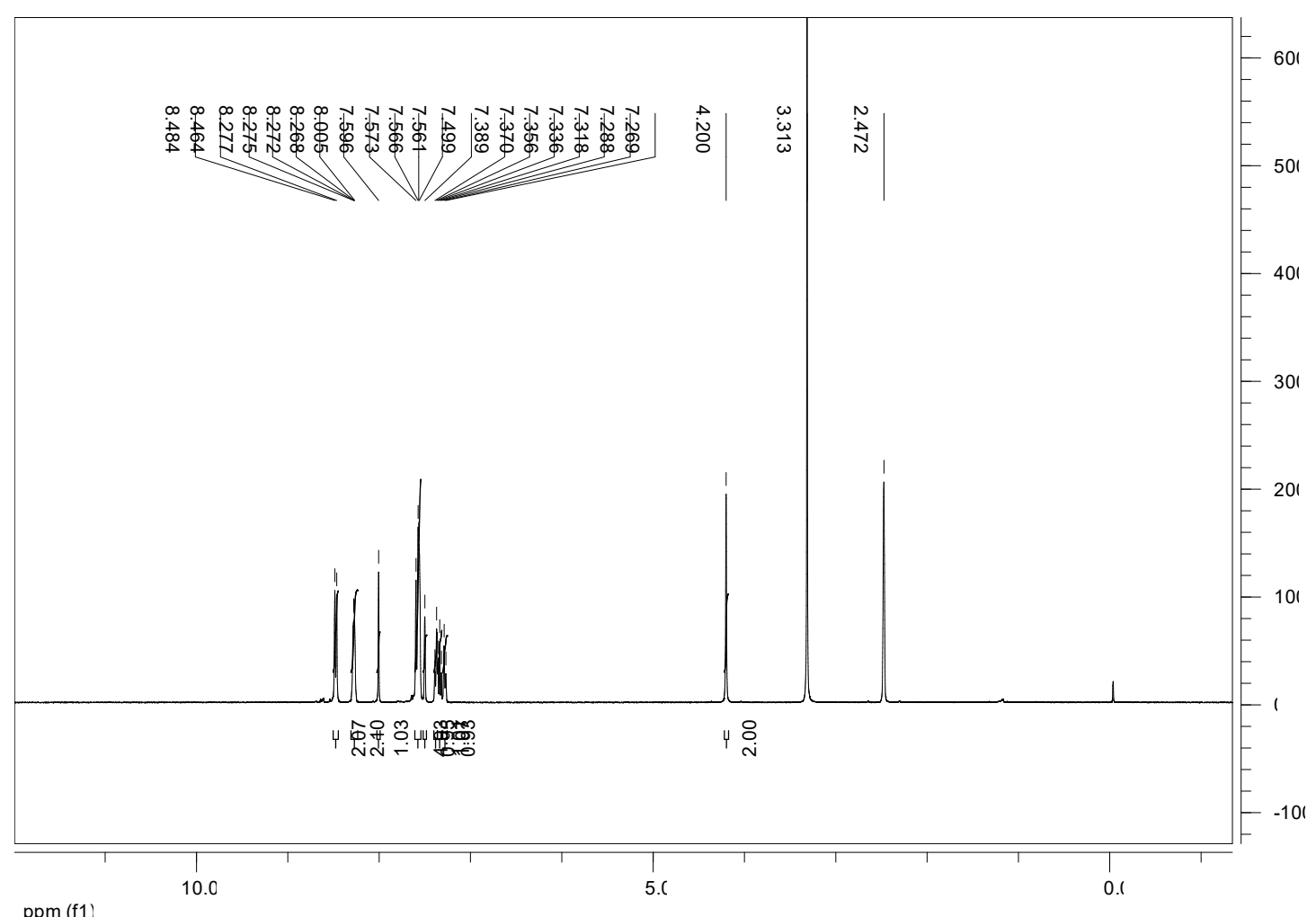

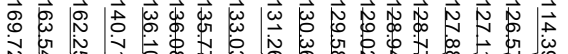

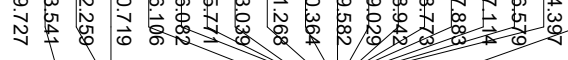
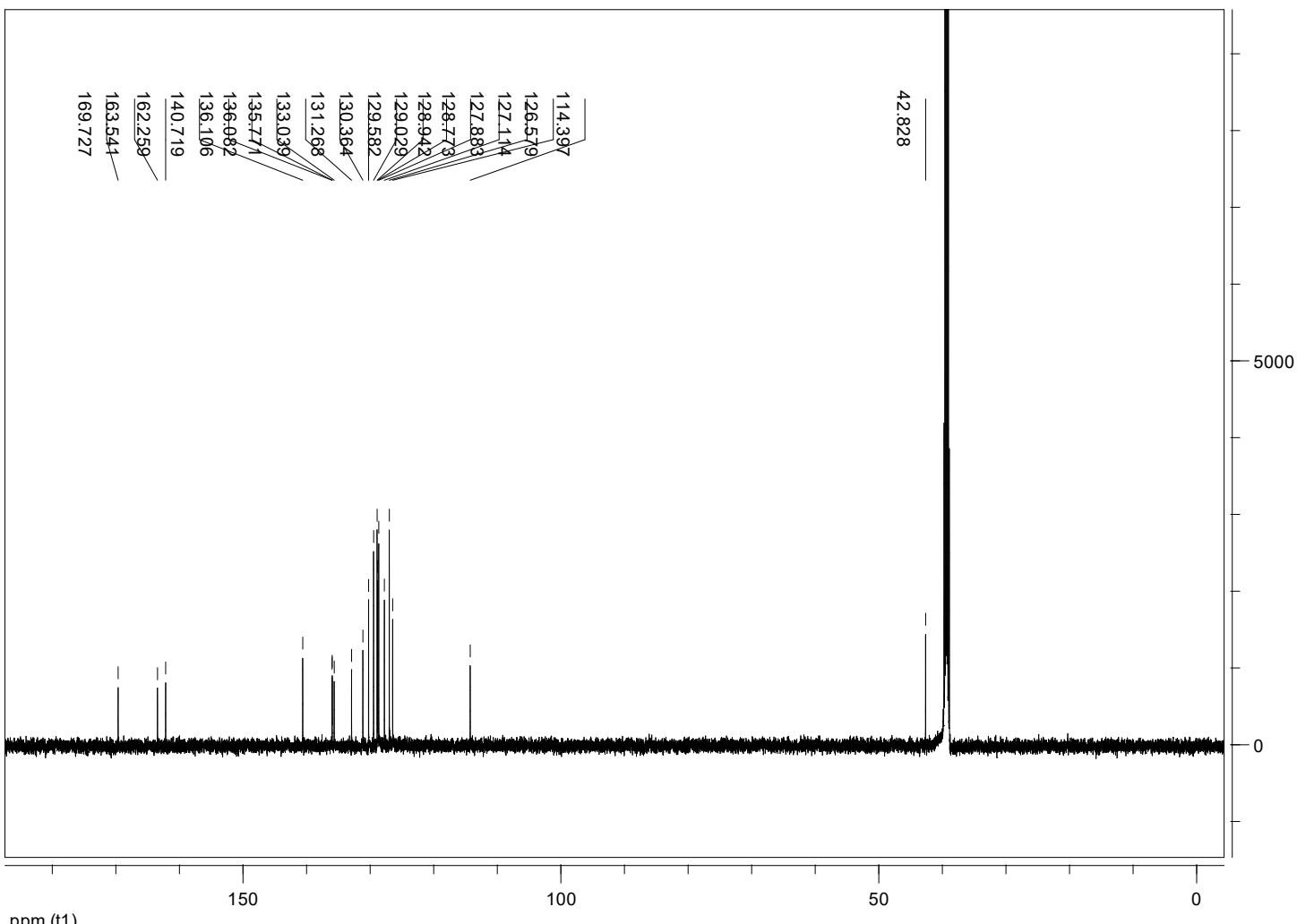
4-(2-bromobenzyl)-2-(4-chlorophenyl)-6-(4-methoxyphenyl)pyrimidine (3j)
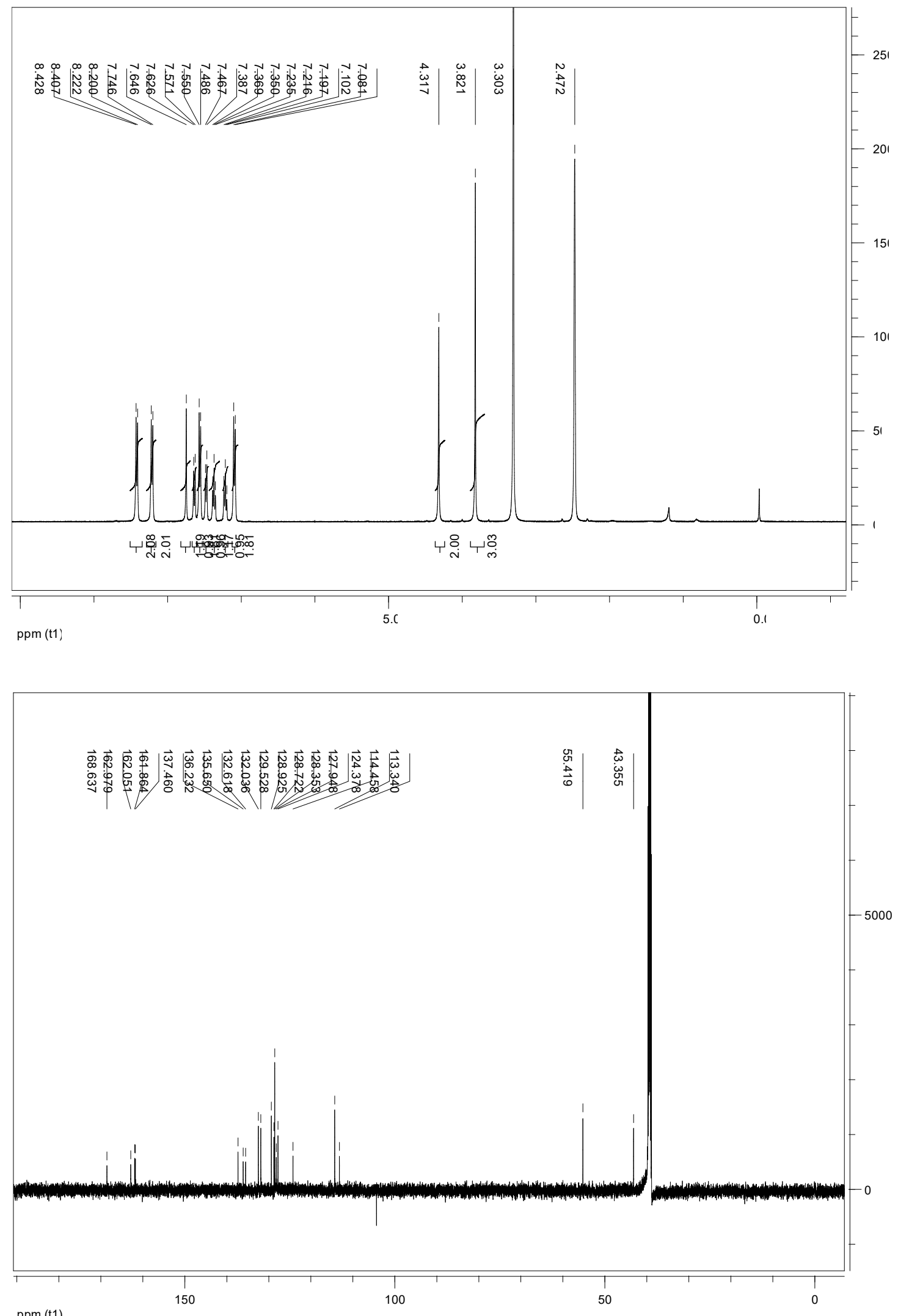

ppm (t1) 
4-(4-chlorobenzyl)-2-(4-chlorophenyl)-6-(p-tolyl)pyrimidine (3k)

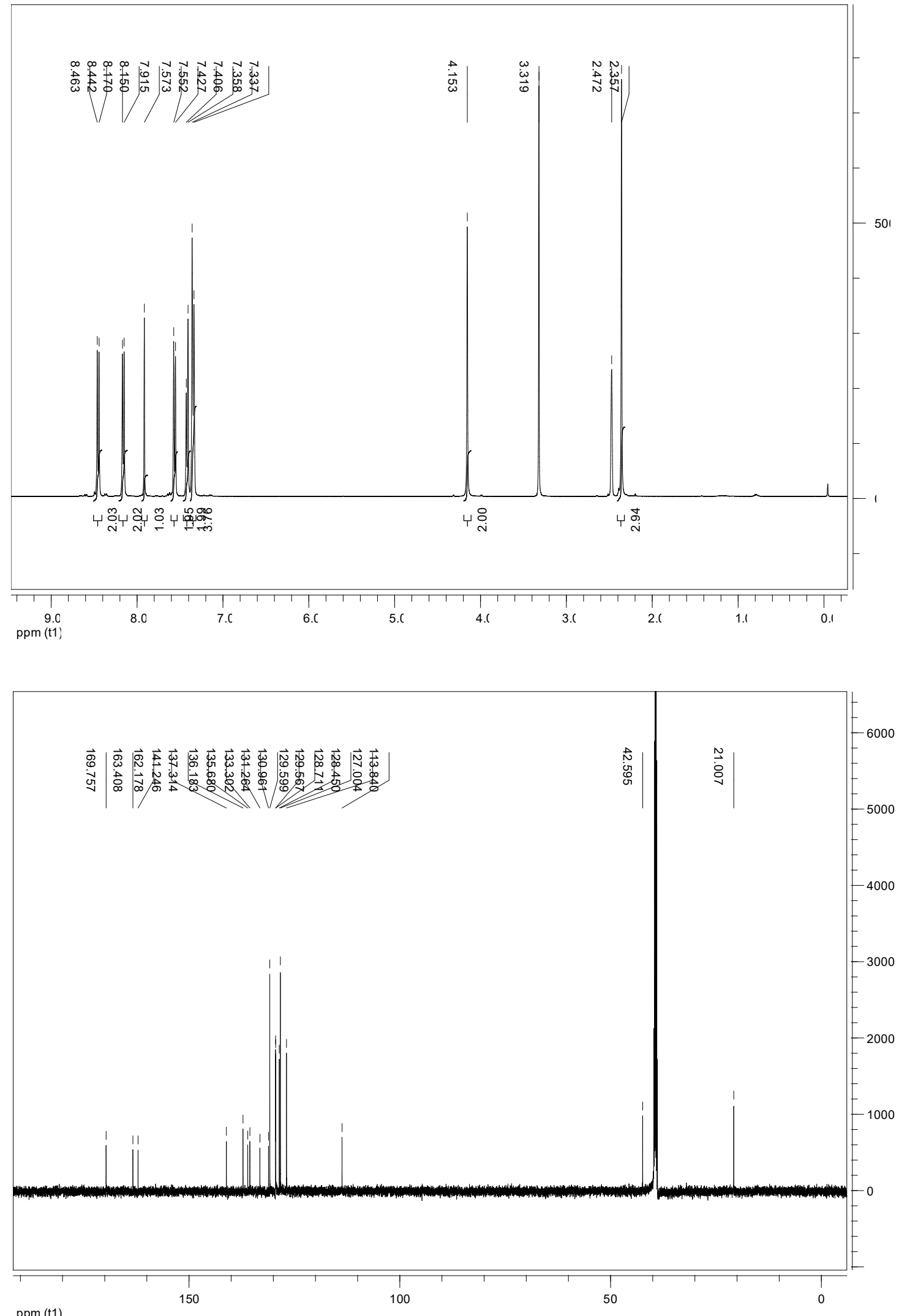


4-(4-chlorobenzyl)-2-(4-chlorophenyl)-6-(4-methoxyphenyl)pyrimidine (3I)
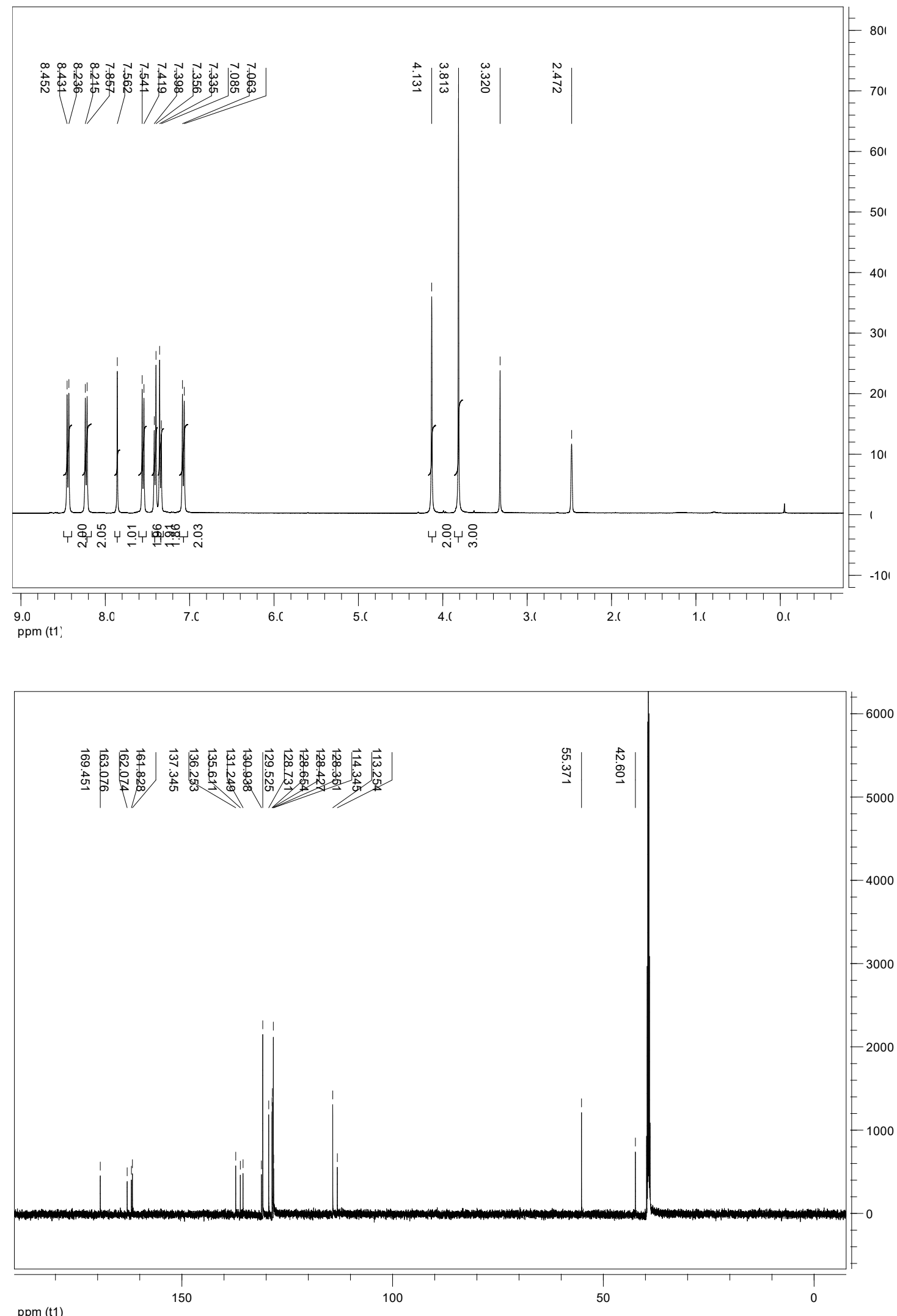
2-(4-chlorophenyl)-4-(4-methylbenzyl)-6-phenylpyrimidine (3 m)

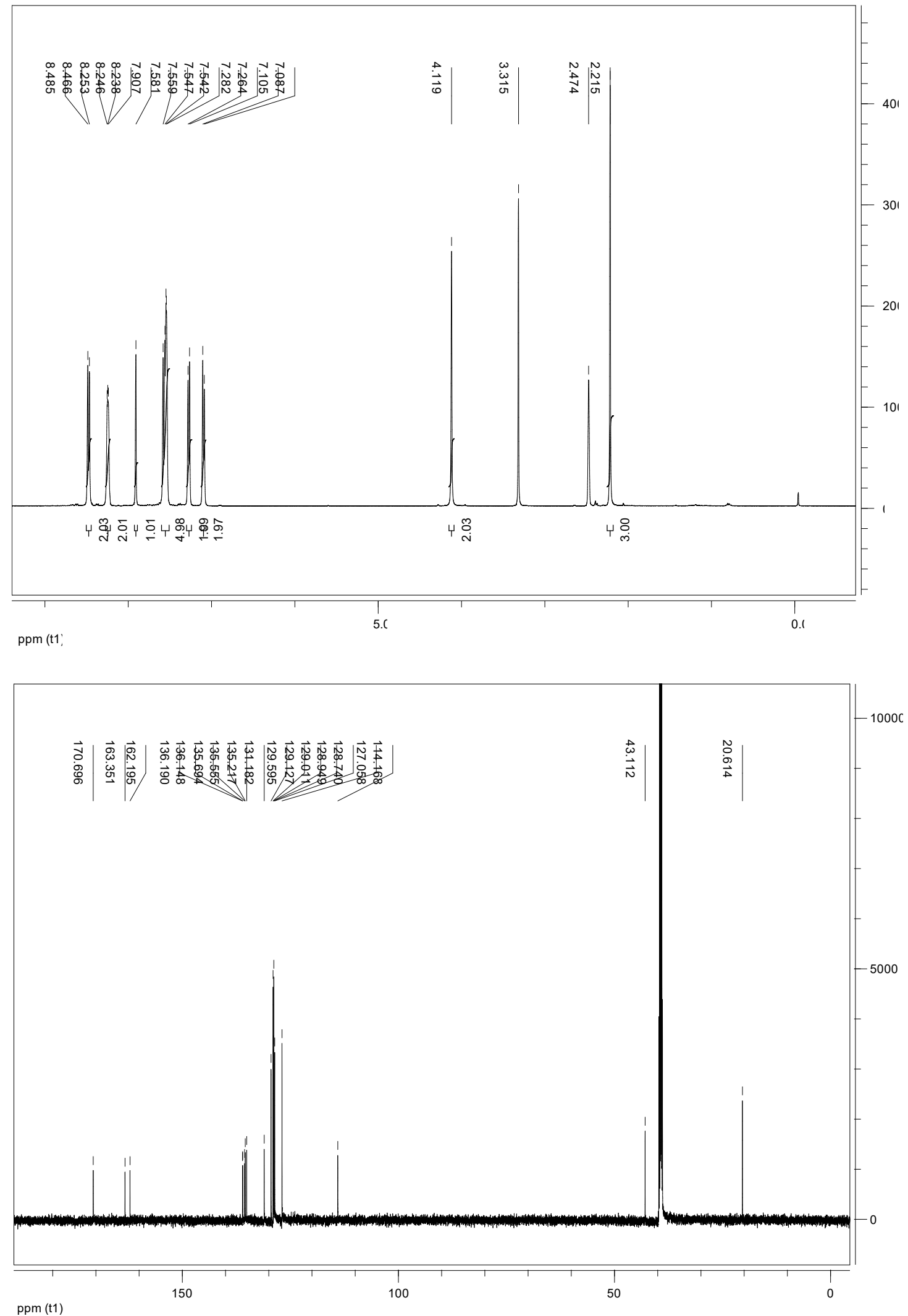


4-(4-bromophenyl)-6-(3-chlorobenzyl)-2-(4-chlorophenyl)pyrimidine (3n)
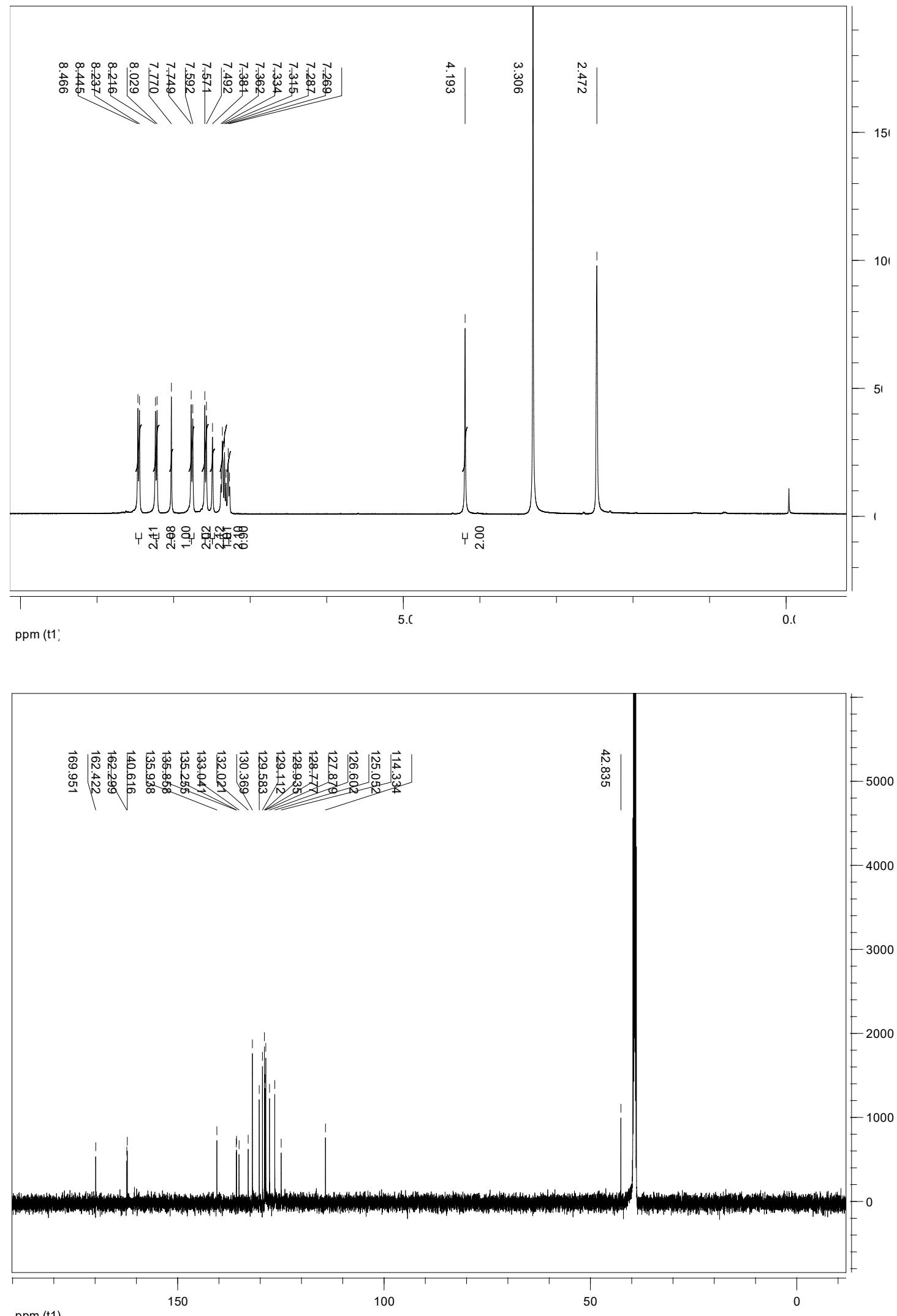

ppm (t1) 
4-(2-chlorobenzyl)-2-(4-chlorophenyl)-6-(p-tolyl)pyrimidine (3o)
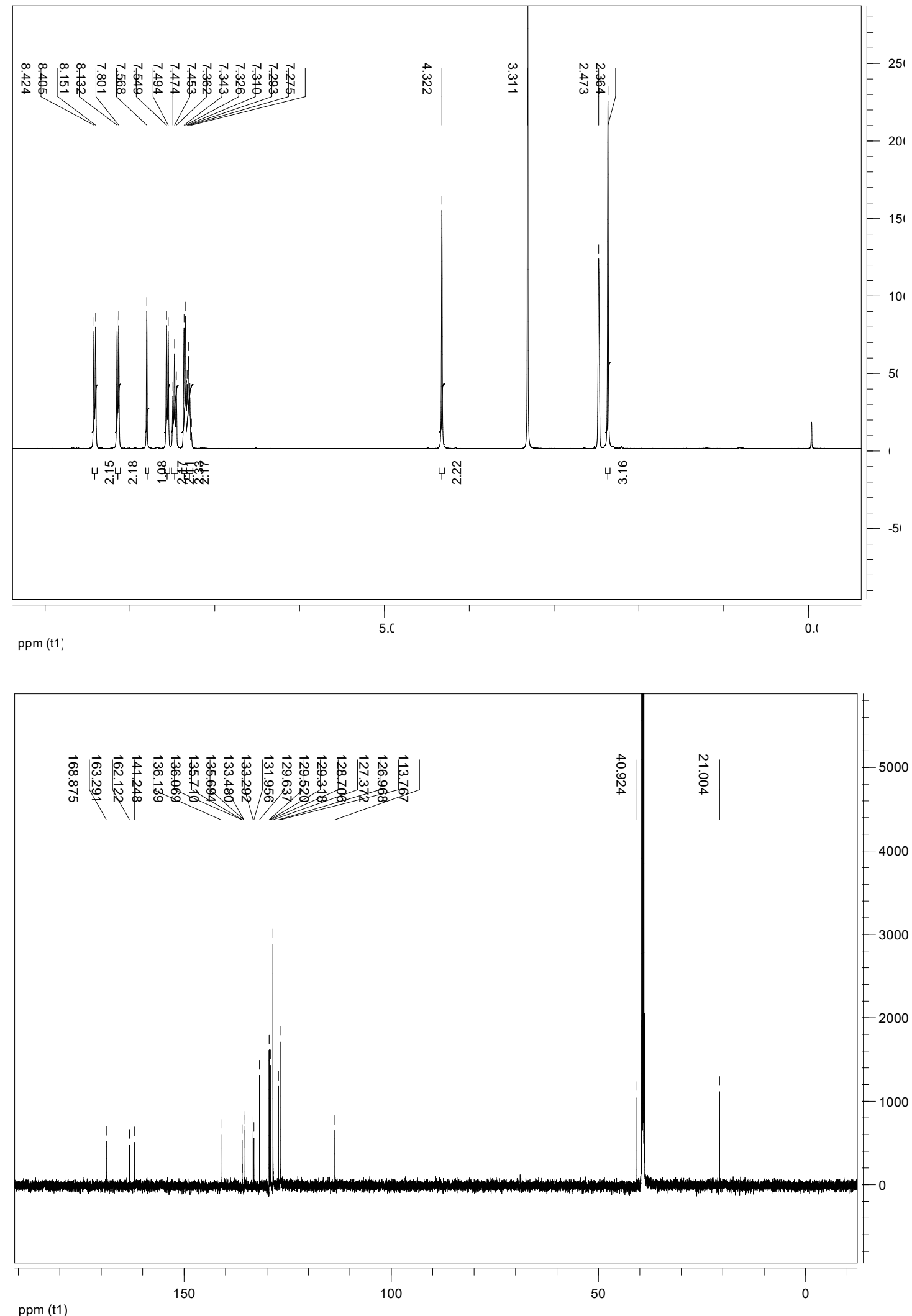
2,4-bis(4-chlorophenyl)-6-(thiophen-3-ylmethyl)pyrimidine (3p)
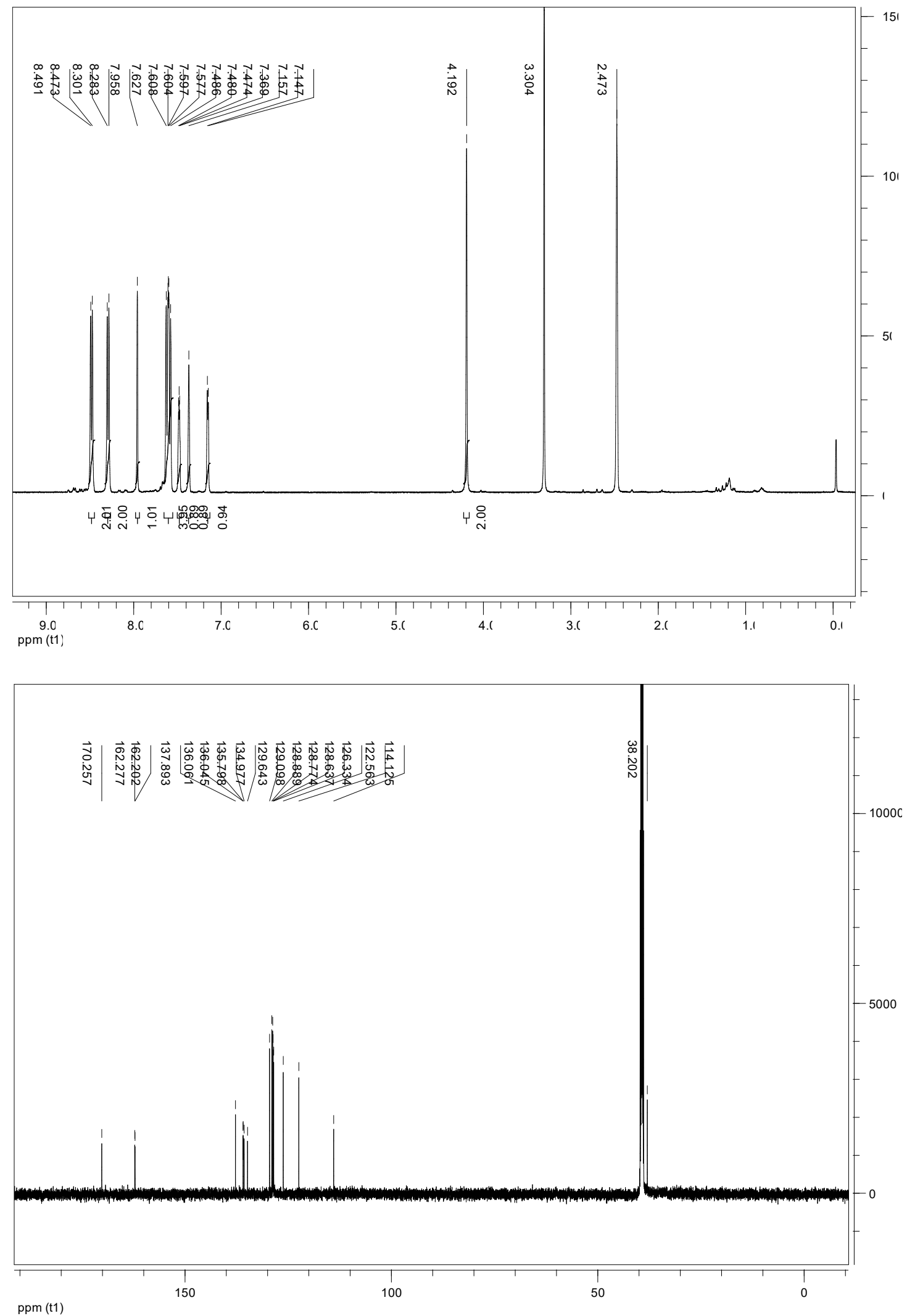

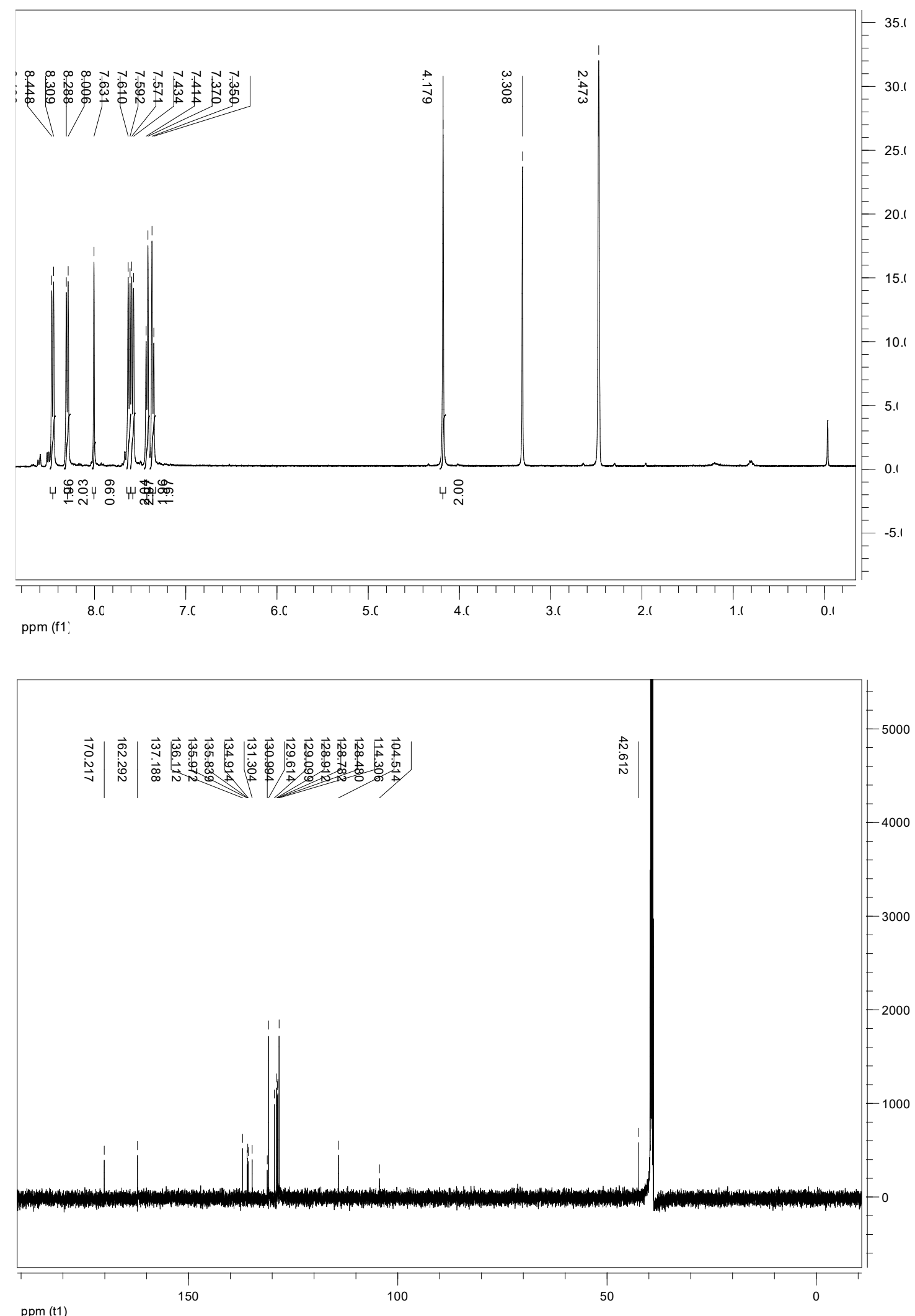
4-(4-bromobenzyl)-6-(4-bromophenyl)-2-(4-chlorophenyl)pyrimidine (3r)

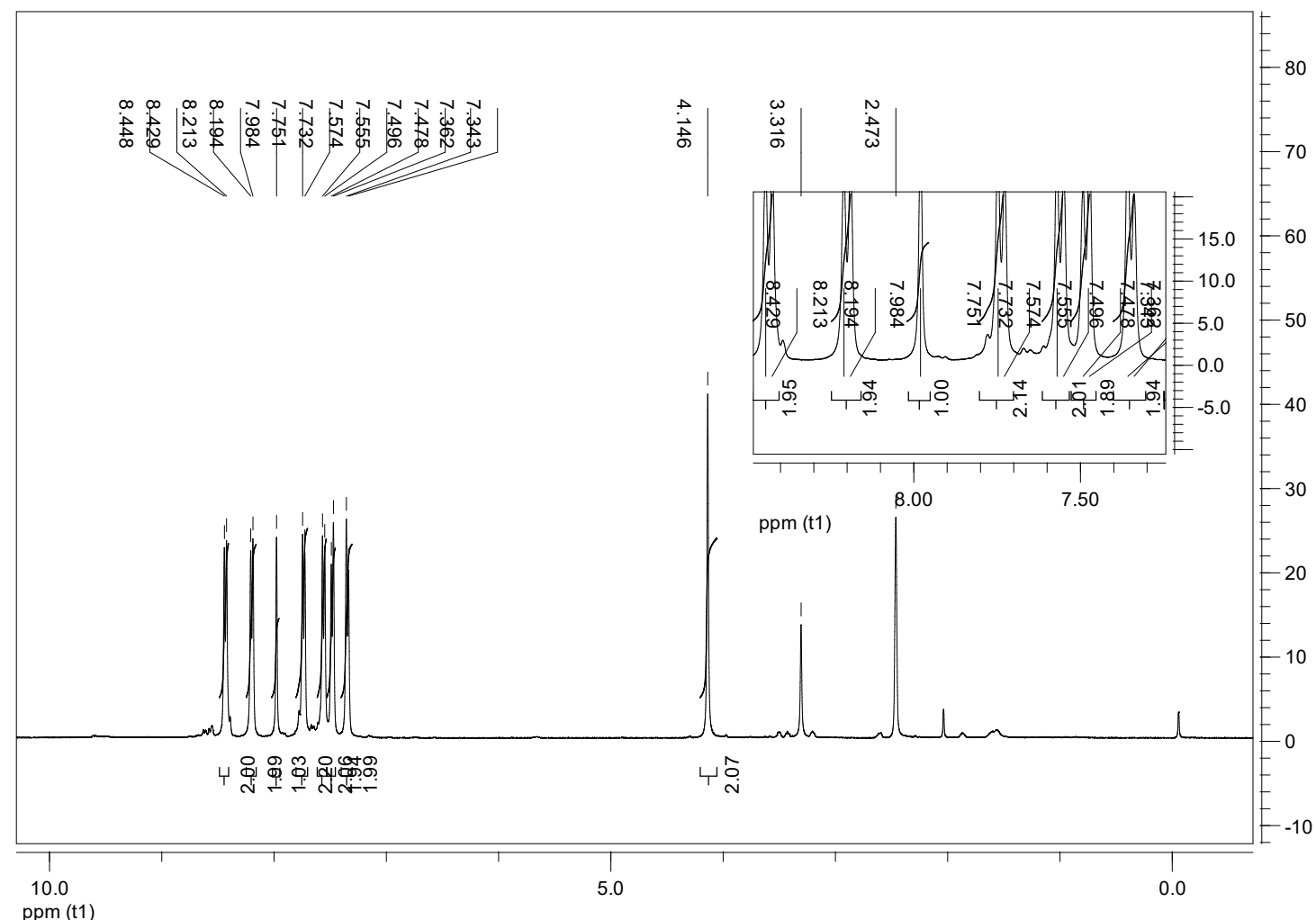

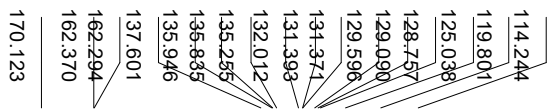

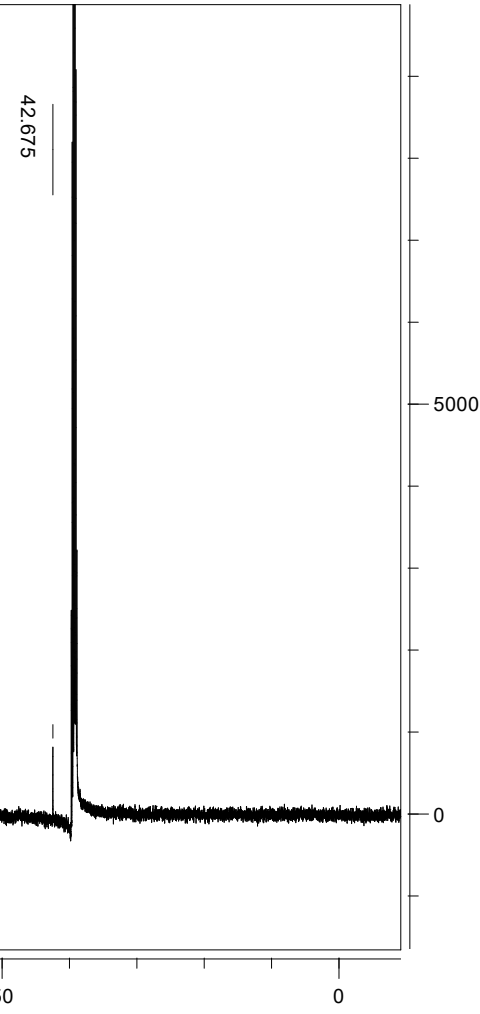

ppm (t1) 
2,4-bis(4-chlorophenyl)-6-(3-phenoxybenzyl)pyrimidine (3s)

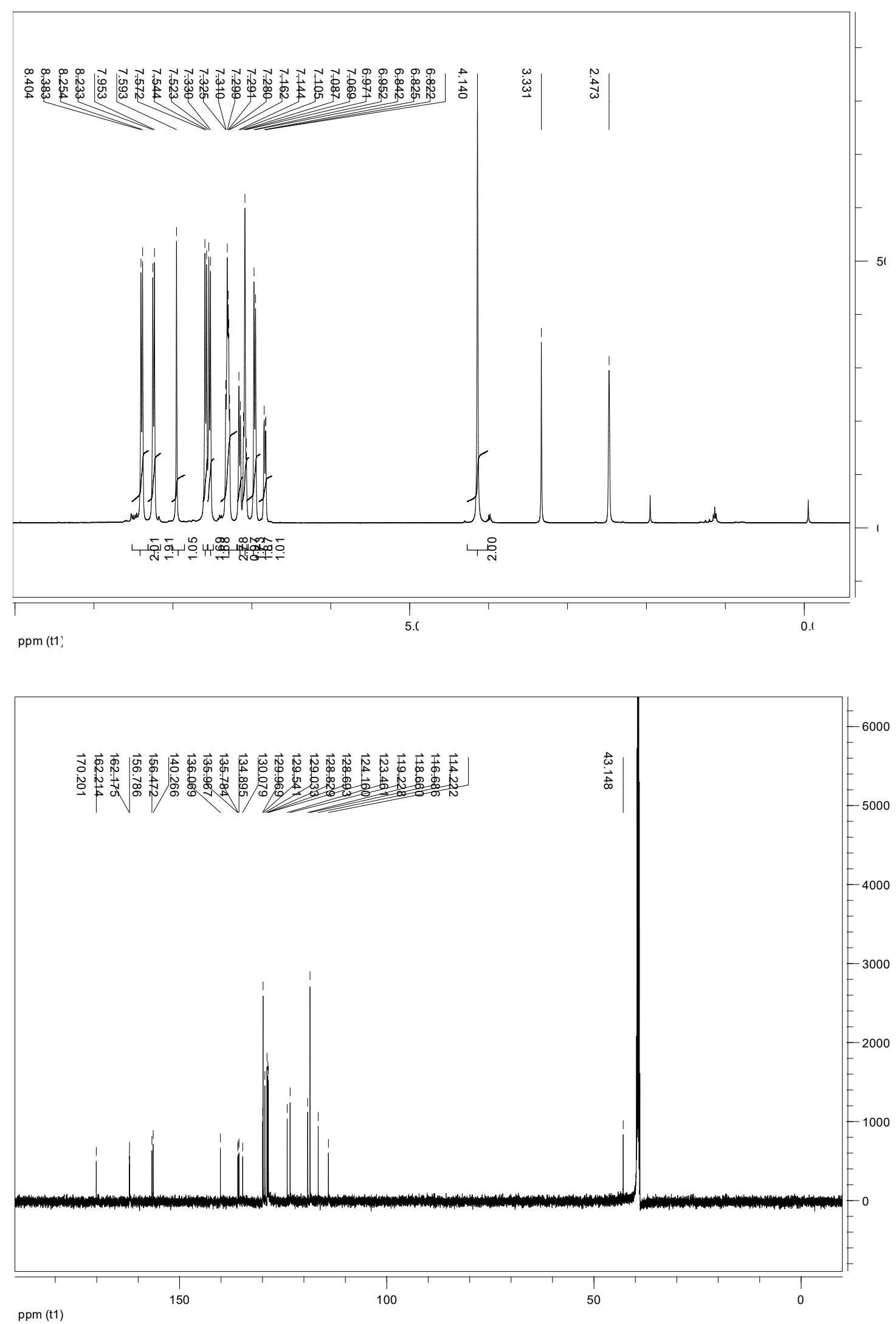


4-(2-bromobenzyl)-2,6-bis(4-bromophenyl)pyrimidine (3t)
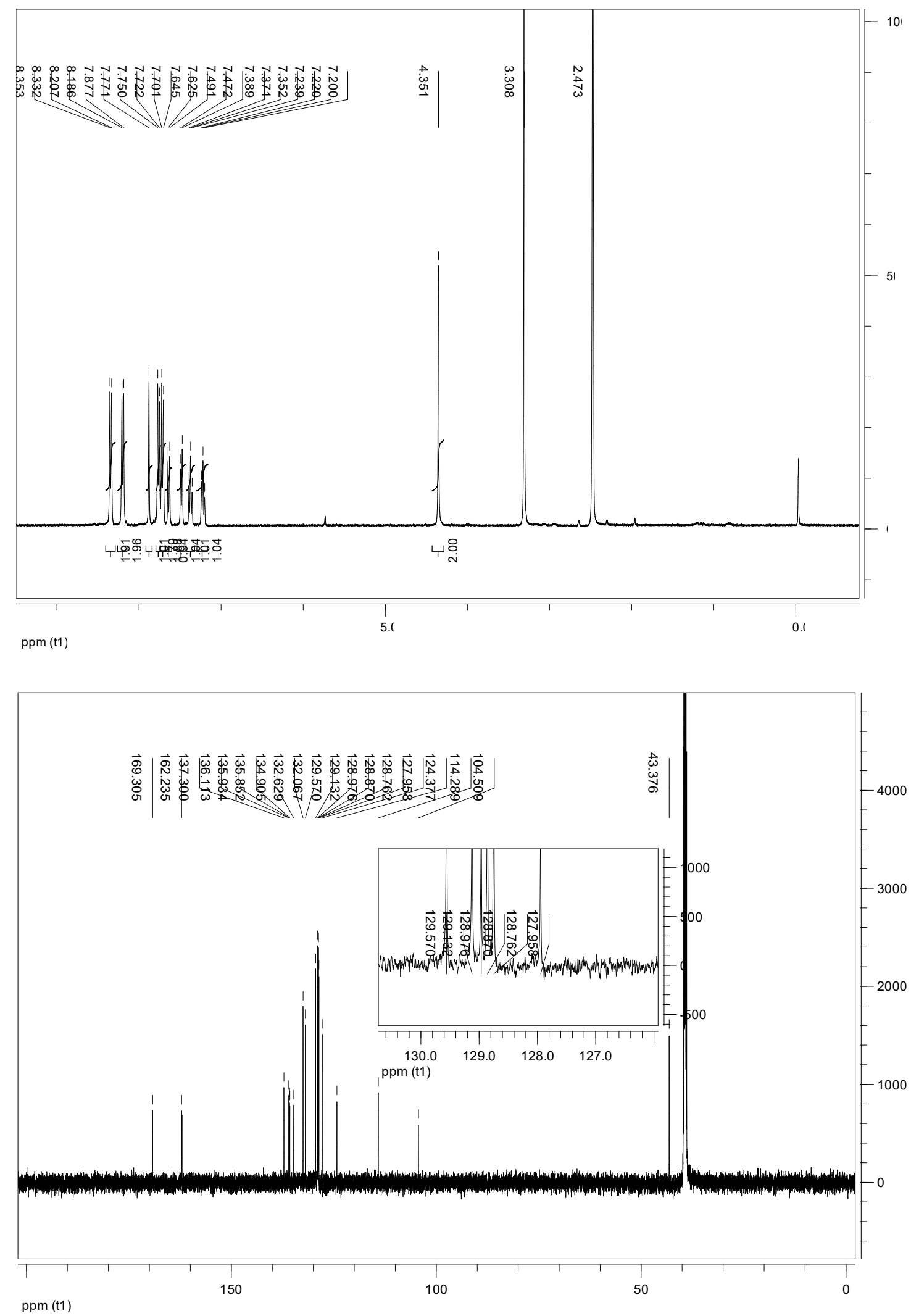
4-(4-bromobenzyl)-2-(4-bromophenyl)-6-(4-chlorophenyl)pyrimidine (3u)

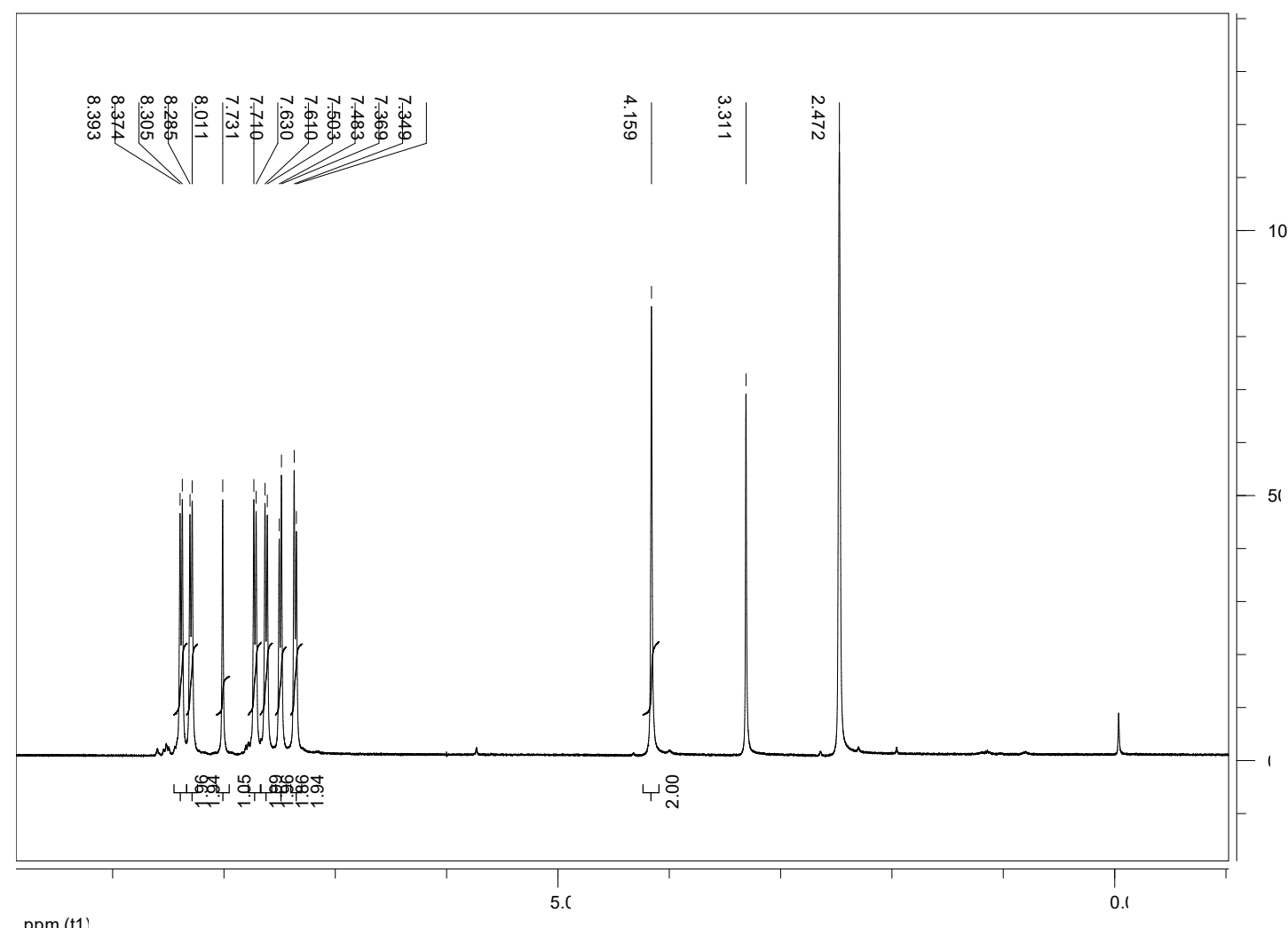

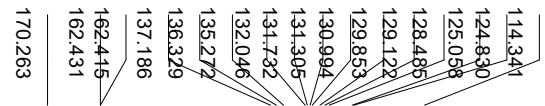

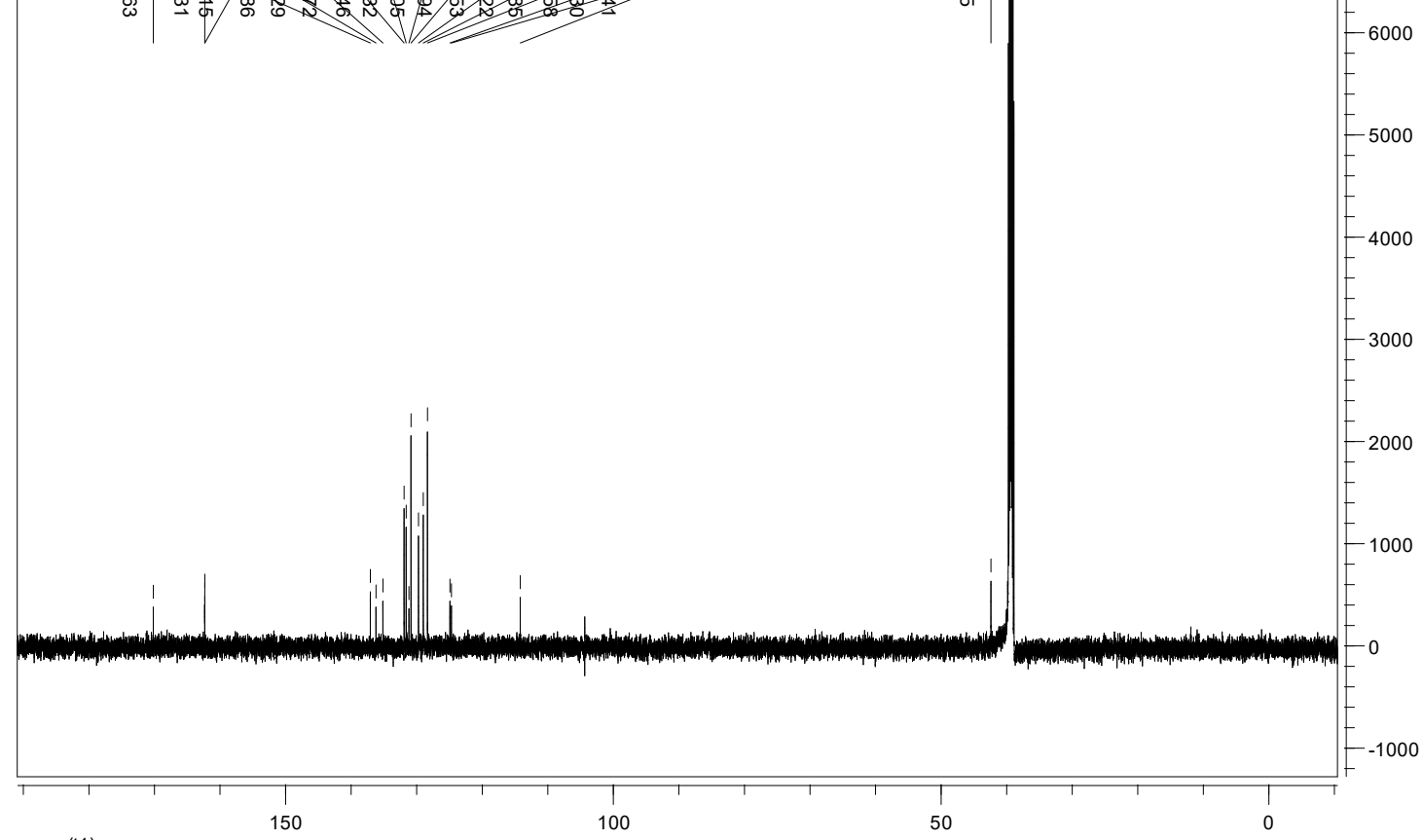

ppm (t1) 
2-(4-bromophenyl)-4-(4-chlorophenyl)-6-(3-phenoxybenzyl)pyrimidine (3v)

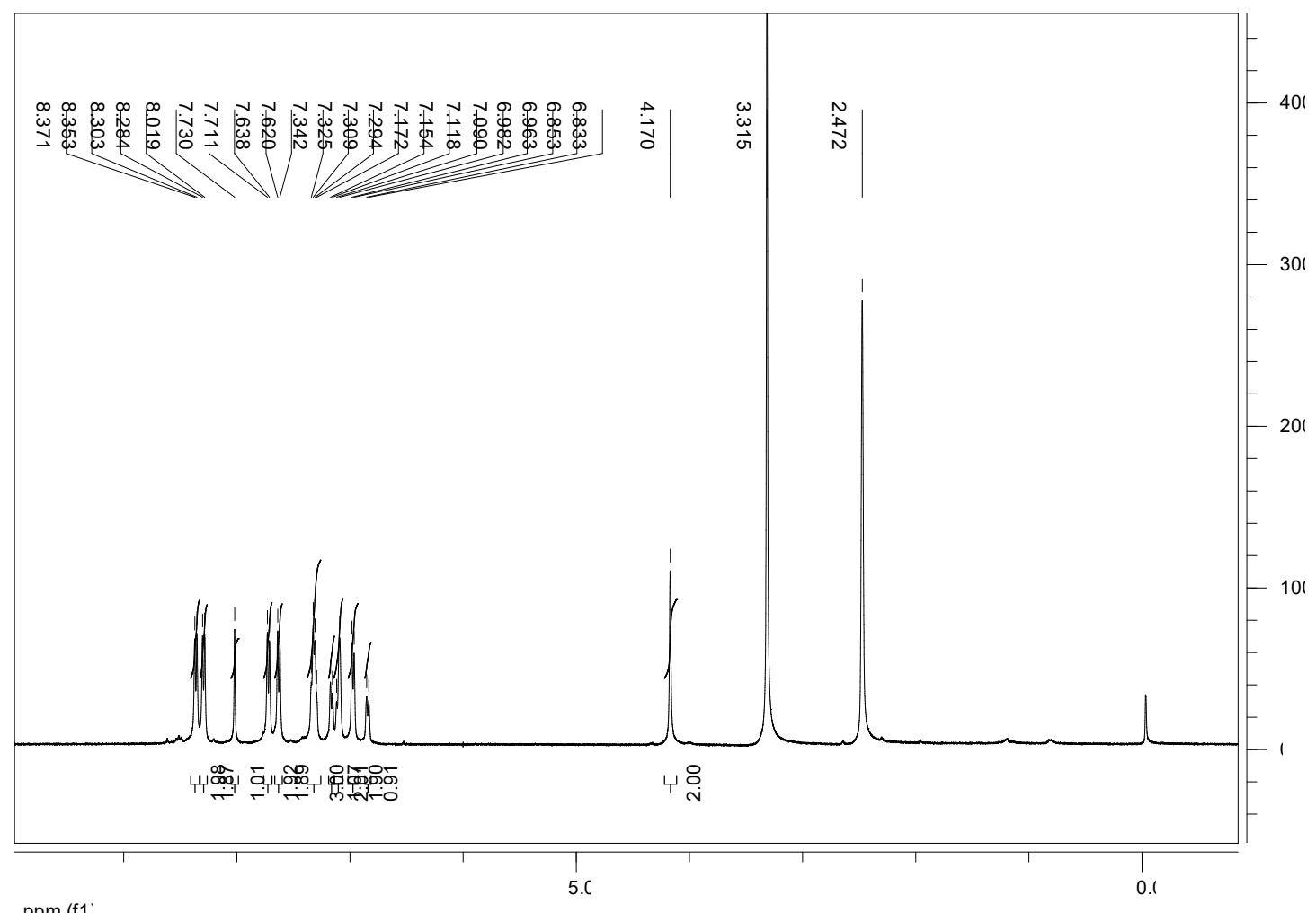

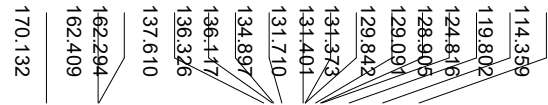

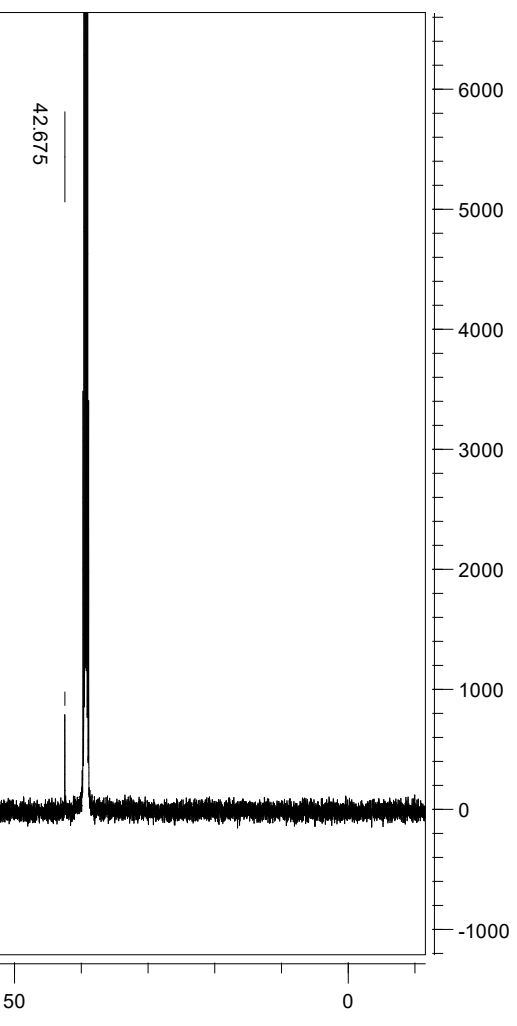

ppm (t1) 
2,4-bis(4-bromophenyl)-6-(4-chlorobenzyl)pyrimidine (3w)

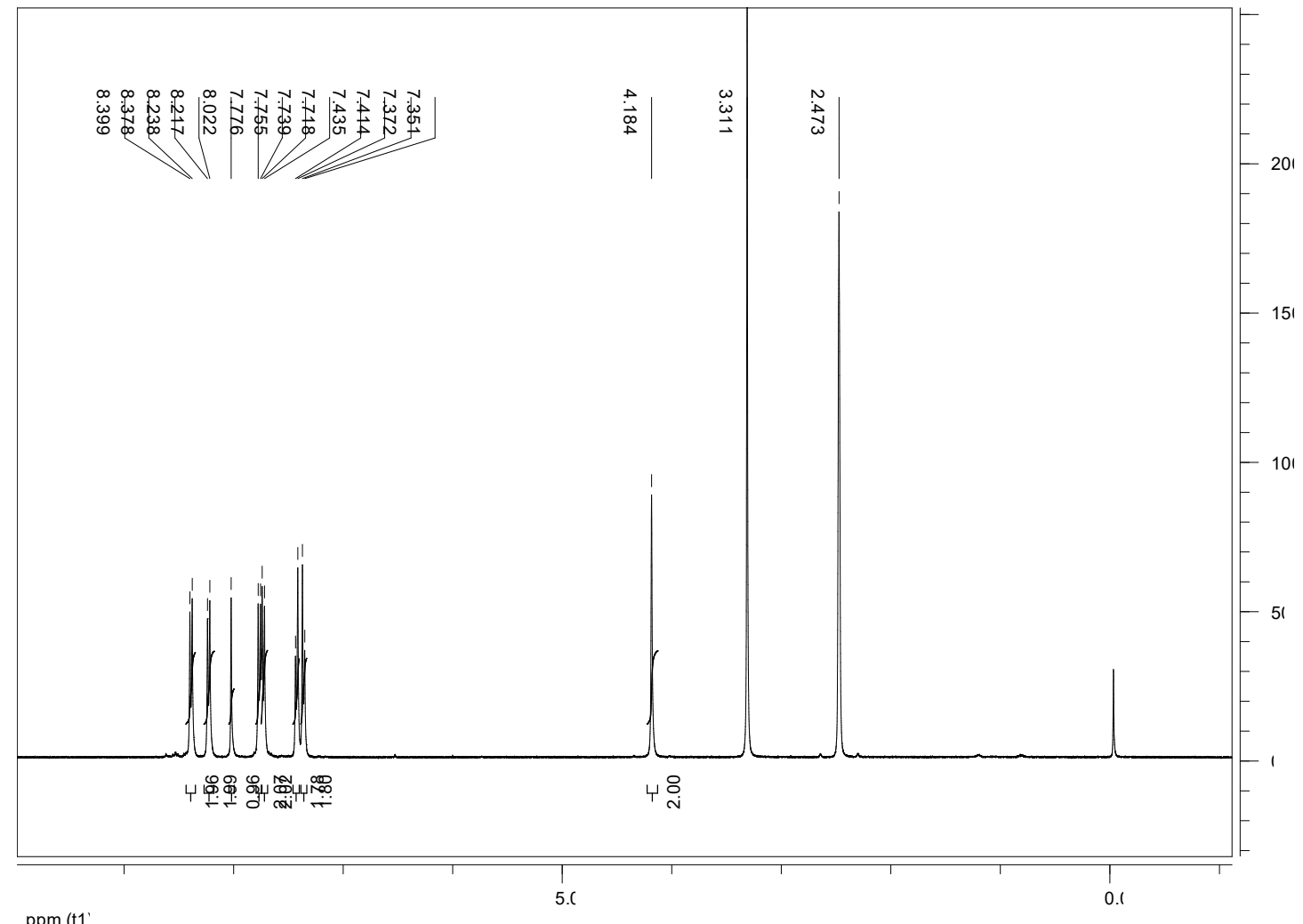

ppm (t1)

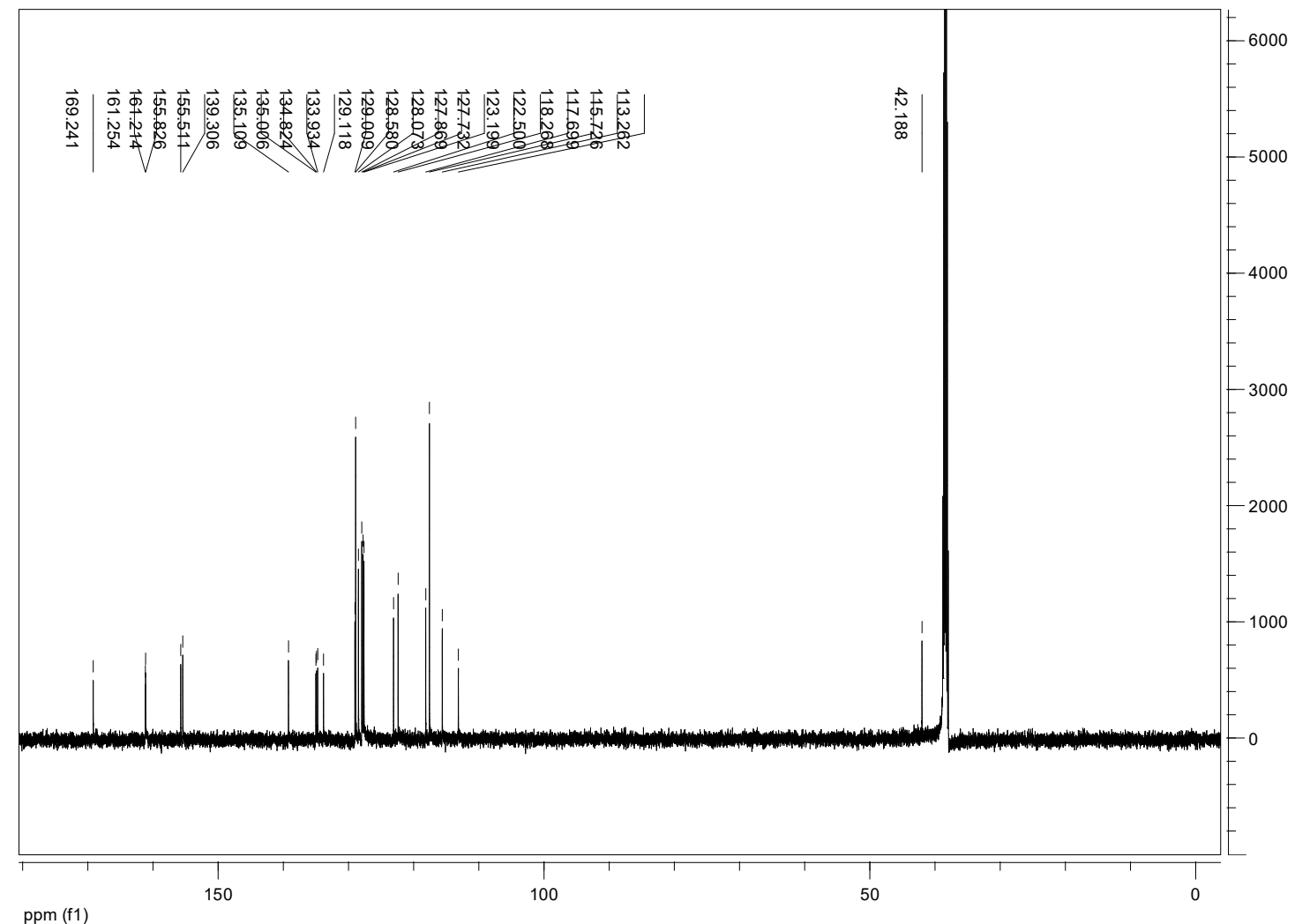


4-(4-bromophenyl)-6-(4-chlorobenzyl)-2-(p-tolyl)pyrimidine (3x)
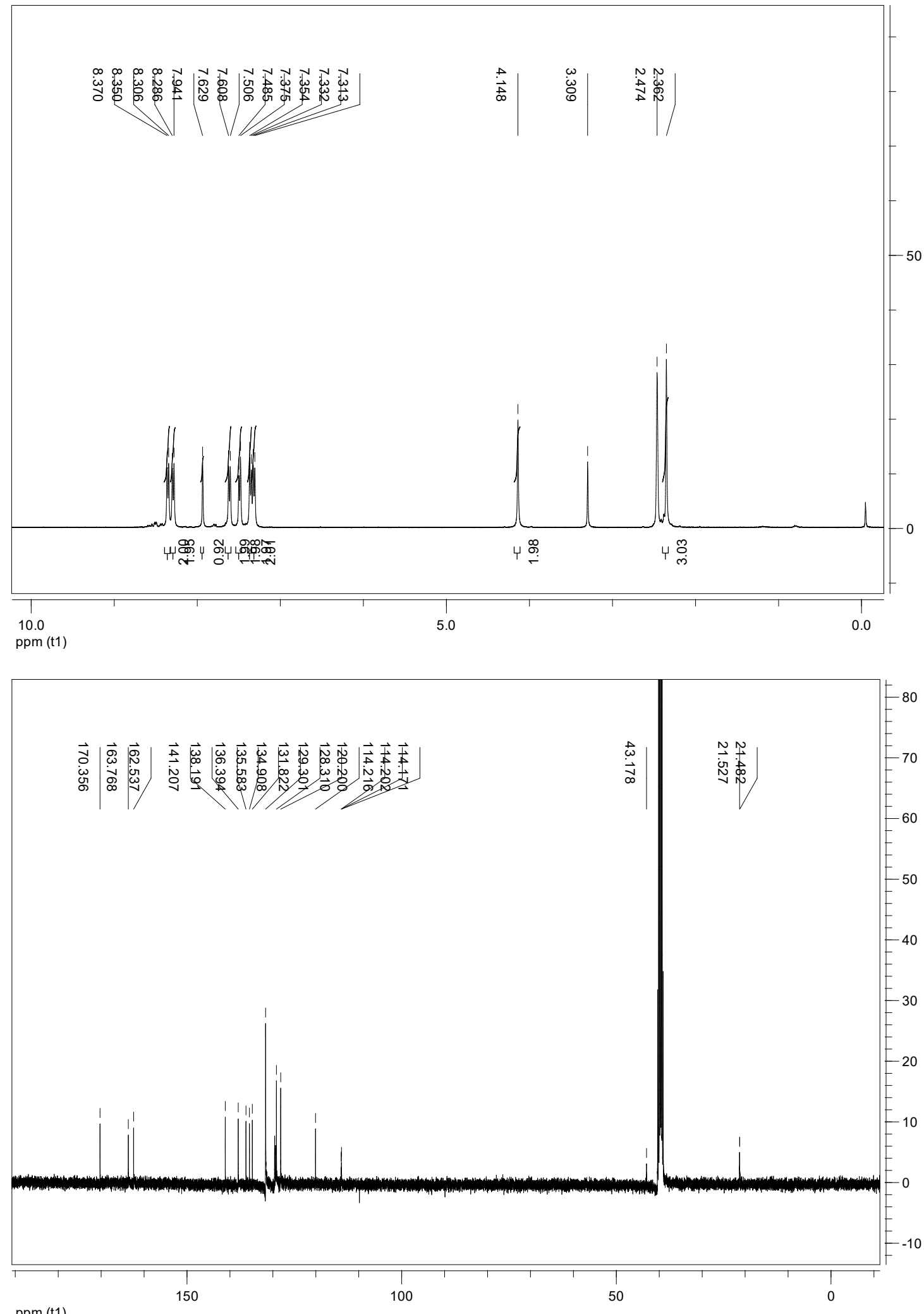
4-(4-chlorobenzyl)-6-(4-chlorophenyl)-2-(p-tolyl)pyrimidine (3y)

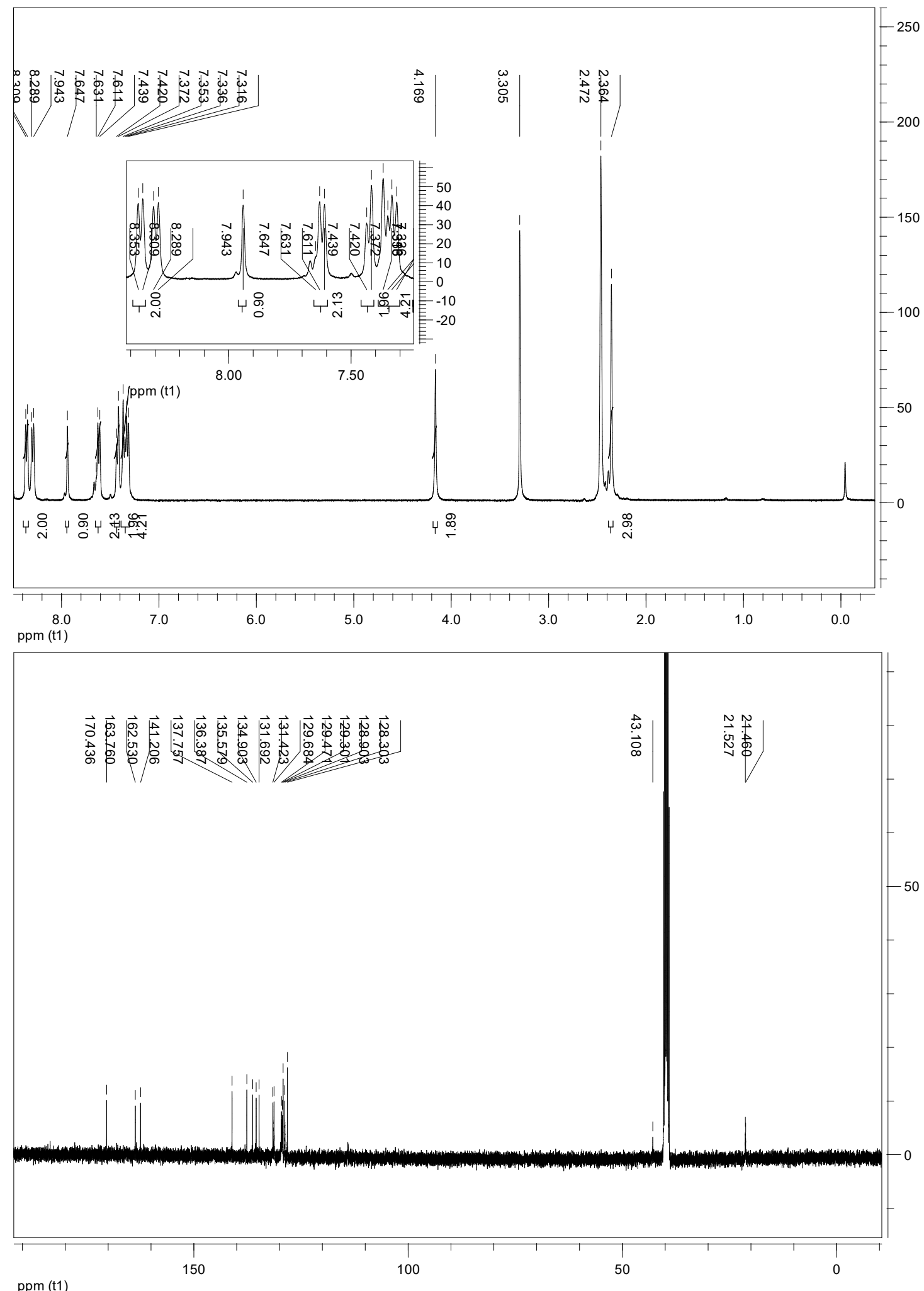


4-(4-Bromophenyl)-2-methyl-6-(4-methylbenzyl)pyrimidine (3z)
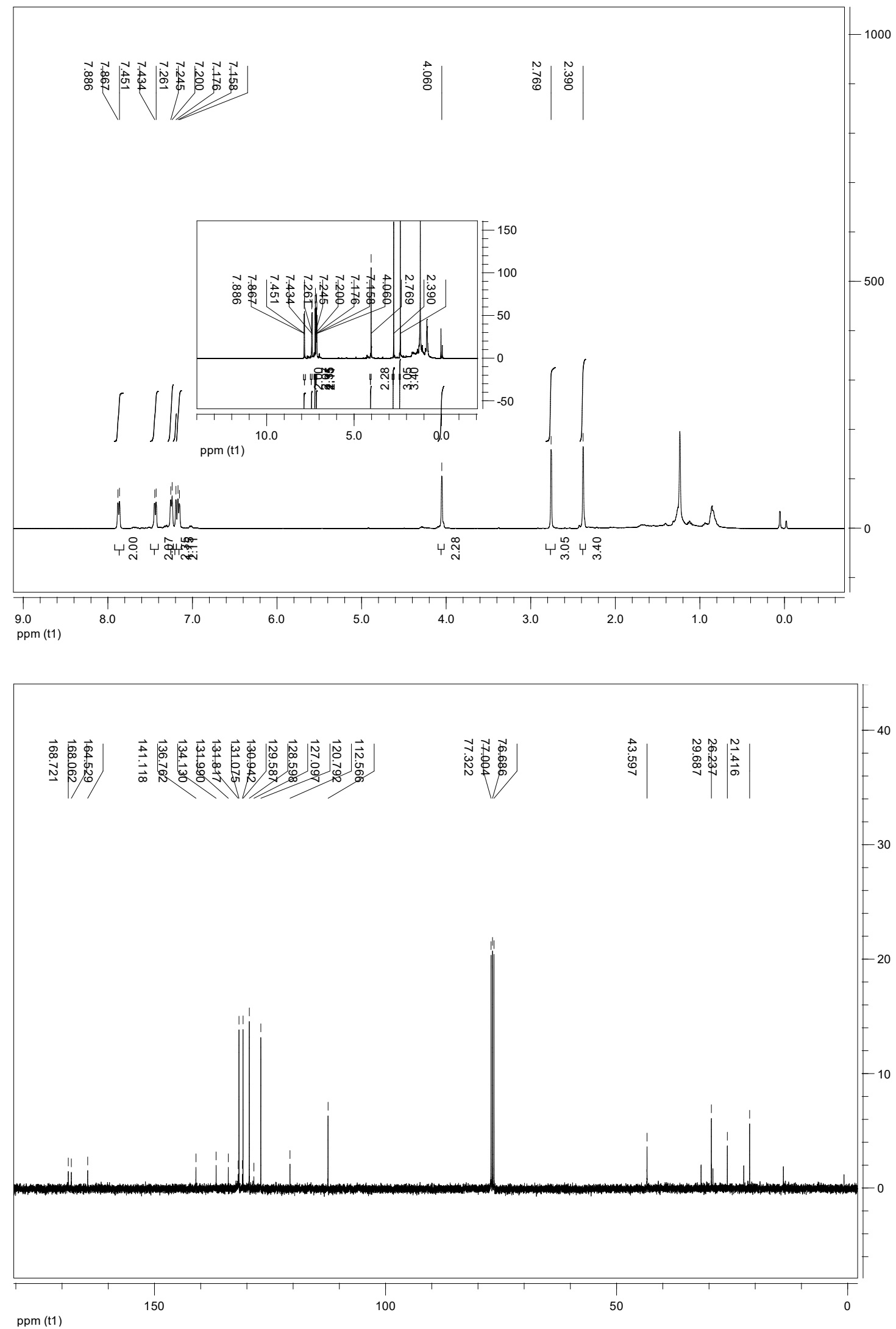
4-(4-Bromobenzyl)-6-(4-chlorophenyl)-2-methylpyrimidine (3aa)
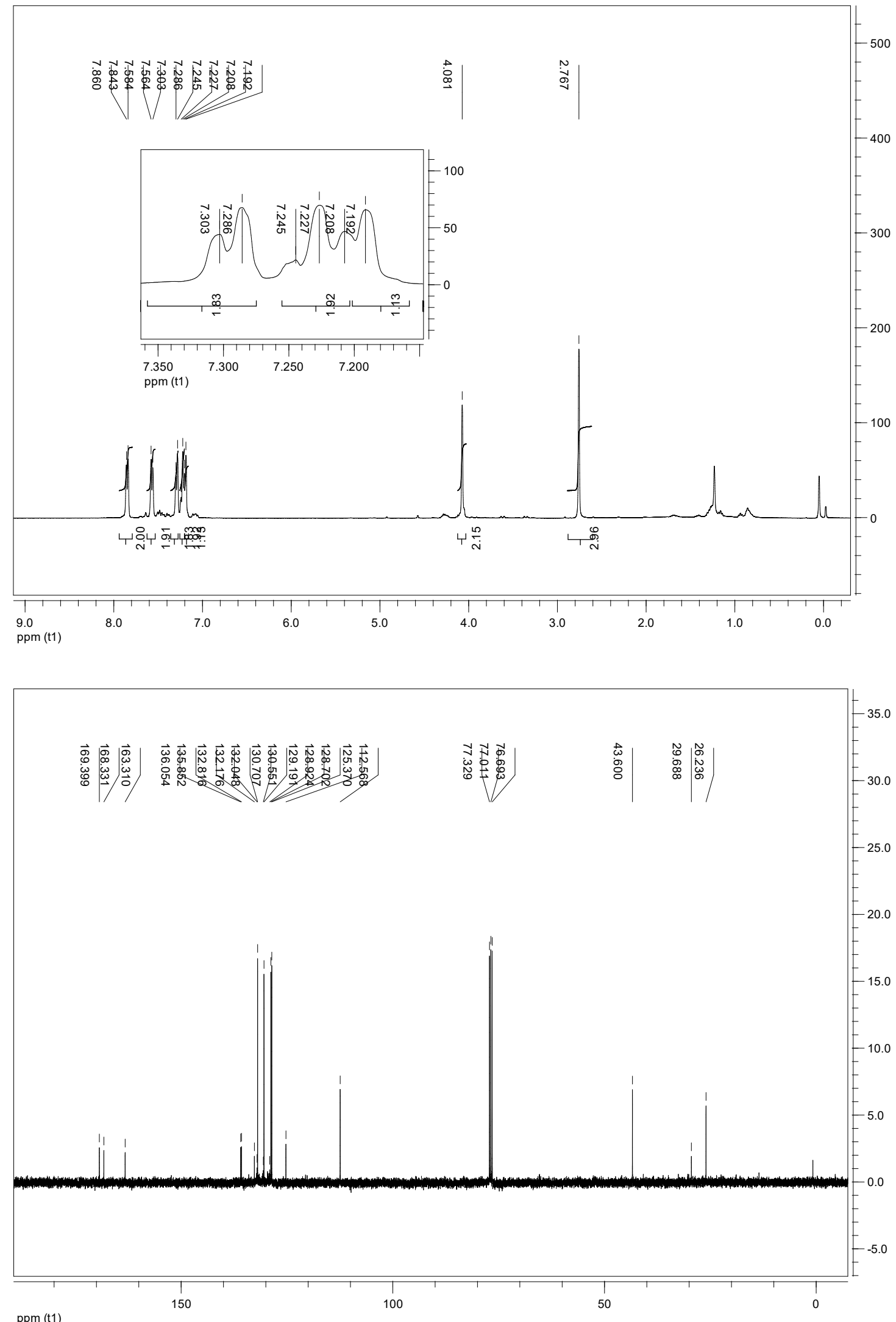


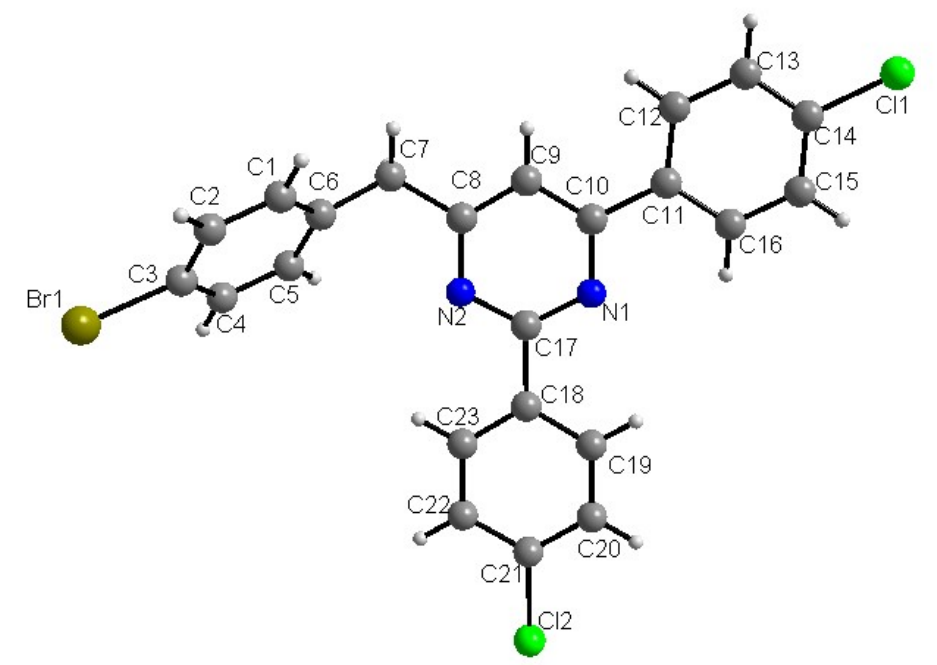

3e (CCDC 1886517)

Figure S1 X-ray crystal structure of compound 3e, non-hydrogen atoms are shown at the 30\% probability level.

Table S1. Crystal data and structure refinement for $\mathbf{3 e}$.

Identification code

Empirical formula

Formula weight

Temperature

Wavelength
$3 \mathbf{e}$

$\mathrm{C}_{23} \mathrm{H}_{15} \mathrm{Br} \mathrm{Cl}_{2} \mathrm{~N}_{2}$

470.17

$298 \mathrm{~K}$

$0.71073 \mathrm{~A}$ 
Crystal system, space group

Unit cell dimensions

Volume

Z, Calculated density

Absorption coefficient

$\mathrm{F}(000)$

Crystal size

Theta range for data collection

Limiting indices

Reflections collected / unique

Completeness to theta $=27.62$

Absorption correction

Monoclinic, $\quad \mathrm{P} 2(1) / \mathrm{c}$

$\mathrm{a}=25.021(4) \mathrm{A} \quad$ alpha $=90 \mathrm{deg}$.

$\mathrm{b}=4.3914(6) \mathrm{A} \quad$ beta $=109.493(4)$ deg.

$\mathrm{c}=19.491(3) \mathrm{A} \quad$ gamma $=90 \mathrm{deg}$.

2018.9(5) $\mathrm{A}^{\wedge} 3$

4, $\quad 1.547 \mathrm{Mg} / \mathrm{m}^{\wedge} 3$

$2.312 \mathrm{~mm}^{\wedge}-1$

944

$0.5 \times 0.2 \times 0.18 \mathrm{~mm}$

0.86 to $27.62 \mathrm{deg}$.

$-32<=\mathrm{h}<=32,-4<=\mathrm{k}<=5,-24<=1<=25$

$19503 / 4695[\mathrm{R}(\mathrm{int})=0.0662]$

$99.1 \%$

Semi-empirical from equivalents 
Max. and min. transmission

Refinement method

Data / restraints / parameters

Goodness-of-fit on $\mathrm{F}^{\wedge} 2$

Final $\mathrm{R}$ indices [I $>2 \operatorname{sigma}(\mathrm{I})]$

$\mathrm{R}$ indices (all data)

Largest diff. peak and hole
1.0000 and 0.7961

Full-matrix least-squares on $\mathrm{F}^{\wedge} 2$

$4658 / 0 / 253$

1.095

$\mathrm{R} 1=0.0639, \mathrm{wR} 2=0.1492$

$\mathrm{R} 1=0.1393, \mathrm{wR} 2=0.1812$

0.439 and -0.440 e. $\mathrm{A}^{\wedge}-3$ 